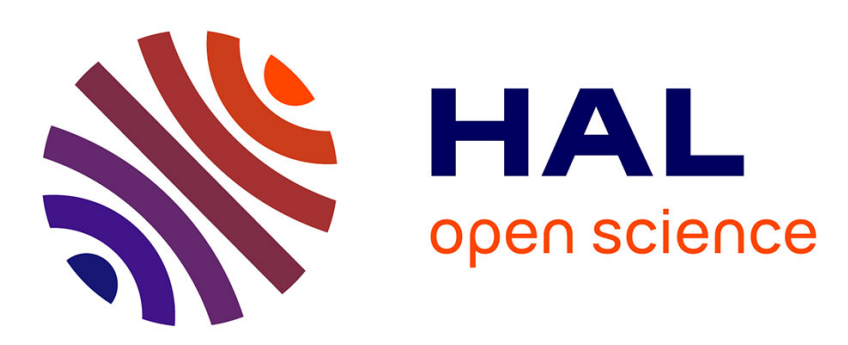

\title{
Absorbing boundary conditions for relativistic quantum mechanics equations
}

Xavier Antoine, Emmanuel Lorin, Julien Sater, François Fillion-Gourdeau, André D. Bandrauk

\section{> To cite this version:}

Xavier Antoine, Emmanuel Lorin, Julien Sater, François Fillion-Gourdeau, André D. Bandrauk. Absorbing boundary conditions for relativistic quantum mechanics equations. Journal of Computational Physics, 2014, 277 (15), pp.268-304. 10.1016/j.jcp.2014.07.037 . hal-01094333

\section{HAL Id: hal-01094333 https://hal.science/hal-01094333}

Submitted on 10 Feb 2016

HAL is a multi-disciplinary open access archive for the deposit and dissemination of scientific research documents, whether they are published or not. The documents may come from teaching and research institutions in France or abroad, or from public or private research centers.
L'archive ouverte pluridisciplinaire HAL, est destinée au dépôt et à la diffusion de documents scientifiques de niveau recherche, publiés ou non, émanant des établissements d'enseignement et de recherche français ou étrangers, des laboratoires publics ou privés. 


\title{
Absorbing Boundary Conditions for Relativistic Quantum Mechanics Equations
}

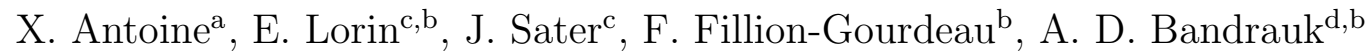 \\ ${ }^{a}$ Institut Elie Cartan de Lorraine, Université de Lorraine, F-54506 Vandoeuvre-ls̀-Nancy Cedex, France \\ ${ }^{b}$ Centre de Recherches Mathématiques, Université de Montréal, Montréal, Canada, H3T 1J4 \\ ${ }^{c}$ School of Mathematics and Statistics, Carleton University, Ottawa, Canada, K1S 5B6 \\ ${ }^{d}$ Laboratoire de chimie théorique, Université de Sherbrooke, Sherbrooke, Canada, J1K 2R1
}

\begin{abstract}
This paper is devoted to the derivation of absorbing boundary conditions for the KleinGordon and Dirac equations modeling quantum and relativistic particles subject to classical electromagnetic fields. Microlocal analysis is the main ingredient in the derivation of these boundary conditions, which are obtained in the form of pseudodifferential equations. $\mathrm{Nu}-$ merical schemes are derived and analyzed to illustrate the accuracy of the derived boundary conditions.
\end{abstract}

Keywords: Microlocal analysis, pseudo-differential operators, absorbing boundary conditions, wave equation, Dirac equation, Klein-Gordon equation, numerical approximation

\section{Contents}

1 Introduction 2

1.1 Introductory remarks $\ldots \ldots \ldots \ldots \ldots \ldots$

1.2 Microlocal approach . . . . . . . . . . . . . . . . . 3

1.3 Organization of the paper $\ldots \ldots \ldots \ldots \ldots$

2 Absorbing Boundary Conditions for the Klein-Gordon Equation 7

2.1 One-dimensional Case . . . . . . . . . . . . . . . . 7

2.1.1 Empirical absorbing conditions . . . . . . . . . . . . 7

2.1.2 Absorbing boundary conditions for Klein-Gordon's equation in 1-d with a constant laser . . . . . . . . . . . . . 8

2.1.3 Absorbing boundary conditions for Klein-Gordon's equation in 1-d

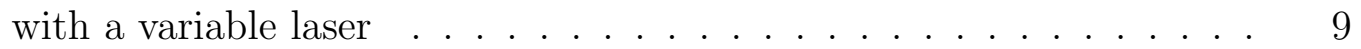

2.2 Two-dimensional TDKE . . . . . . . . . . . . . . . . . . . . . . 12

Email addresses: Xavier.Antoine@univ-lorraine.fr (X. Antoine), elorin@math.carleton.ca (E. Lorin), julien.sater@hotmail.com (J. Sater), filliong@CRM. UMontreal.ca (F. Fillion-Gourdeau), andre. bandrauk@usherbrooke.ca (A. D. Bandrauk) 
2.2 .1 Laser-free TDKGE in 2-d . . . . . . . . . . . . . . . . . 12

2.2 .2 Laser-particle TDKGE in 2 -d . . . . . . . . . . . . . 15

3 Absorbing Boundary Conditions for Dirac Equation in 2-d 22

3.1 Dirac equation . . . . . . . . . . . . . . . . . . . . . . . 22

3.2 Laser-particle TDDE in 2-d . . . . . . . . . . . . . . 23

3.3 Application to 1-d Dirac equation . . . . . . . . . . . . . . . 34

4 Other techniques $\quad 36$

4.1 Volkov approach . . . . . . . . . . . . . . . . . 36

4.2 Basic transformations on TDKGE and TDDE . . . . . . . . . . 39

5 Numerical Simulations $\quad 40$

5.1 Discretization for TDKGE . . . . . . . . . . . . . . . . . 40

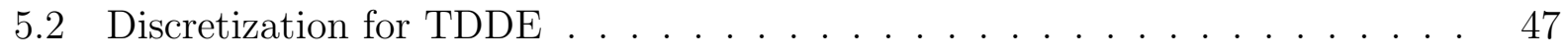

$\begin{array}{lll}6 & \text { Conclusion } & 49\end{array}$

\section{Introduction}

\subsection{Introductory remarks}

This paper deals with the derivation of transparent and absorbing boundary conditions (TBC, ABC) for the time dependent Dirac (TDDE) and time dependent Klein-Gordon equations (TDKGE) modeling the interaction of classical electromagnetic fields with quantum particles. Both equations are relativistic version of the Schrödinger equation for spin-0 particles (TDKGE) and spin $\pm 1 / 2$ particles (TDDE). TDDE is an order 1 linear system of 4 equations, while the TDKGE is an order 2 linear equations. When dealing with the discretization of complex wave-like equations, such as the linear or nonlinear wave, Schrödinger, Maxwell, Klein-Gordon, Dirac equations, a crucial question is the reduction of the size of the computational domain. Reducing the computational domain allows to simultaneously reduce data storage and computational complexity. However, when local or nonlocal internal waves reach the boundary of the domain where too simple boundary conditions (e.g. Neumann, Dirichlet) are imposed, they may be reflected so that spurious waves are generated in the domain and interact with physical waves. Avoiding this issue is an old problem that many papers have addressed, such as famous Engquist and Majda's paper [10], but also [16] and more recently $[2,4]$. In this paper, we apply two techniques to rigorously derive TBC\&ABC for these 2 equations. The proposed approaches are derived using microlocal analysis and pseudodifferential operators in the spirit of $[17,18,26,28]$ and more specifically [22]. Some original techniques that we will use were proposed by $[3,4,5]$ in the framework of Maxwell's equations and linear and nonlinear Schrödinger equations. The general approach presented by Barucq et al. is now become standard and is applied to several kinds of equations, such as evolution equations with cubic nonlinearity [30]. This paper is an instance of these approaches in the framework of relativistic quantum mechanics for laser-particle TDDE and 


\section{TDKGE.}

The complexity of the solution to laser-molecule TDDE in realistic situations is well-known. For instance, when a intense femtosecond laser pulse $\left(\sim 10^{15} \mathrm{~s}\right)$ of frequency $\omega_{0}$ interacts with a molecule, it involves multiscale phenomena, in time and space (zitterbewegung $\sim 10^{-21} \mathrm{~s}$, [29]), in frequency (high order harmonics generation, often beyond $100 \omega_{0}$ ), etc. As a consequence a fine discretization necessitates high order methods, adaptive mesh refinement, etc $[11,12,23]$. The price to pay is naturally a huge computational complexity of the involved methods.

In this paper, we derive ABC for TDDE and TDKGE on a circle, although the technique can be extended to more general smooth surfaces.

\subsection{Microlocal approach}

General Principle. The goal of this paragraph is to recall some important notions and ideas about the derivation of TBC/ABC using microlocal analysis in order to make the paper accessible to non-specialists. For details, we refer to $[17,18,26]$ for the theory and to $[4,5]$ for applications to the linear and nonlinear Schrödinger equation and Maxwell's equations [2]. The framework that we consider here is as follows. Let a 2-d partial differential equation (or system) in $\mathbb{R}^{2} \times[0, T]$

$$
P\left(x, y, t, \partial_{t}, \partial_{x}, \partial_{y}\right) \mathbf{u}(x, y, t)=\mathbf{0}, \quad \mathbf{u}(x, y, 0)=\mathbf{u}_{0}(x, y),
$$

where $P=P\left(x, y, t, \partial_{t}, \partial_{x}, \partial_{y}\right)$ belongs to the set of order $m$ pseudo-differential operators, denoted by $O P S^{m}$. Time $T>0$ is chosen such that system (1) is well-posed. Operator $P$ has a symbol which is denoted by $p \in S^{m}$ (which is equal to $\operatorname{det}(\mathcal{P})$ where $\mathcal{P}=\sigma(P)$ if $(1)$ is a system).

We now recall some basic definitions and facts about wavefronts, bicharacteristic strips, and operator factorizations. Details can be found in the classical references [17], [18], [1], [28]. For $\mathbf{u} \in \mathcal{D}^{\prime}\left(\mathbb{R}^{2} \times \mathbb{R}_{+}\right)$the wavefront $W F(\mathbf{u})$, is defined as the set of singularities in real $\mathbf{x}=(x, y, t)$ and co-variable spaces $\boldsymbol{\zeta}=(\xi, \eta, \tau)$ that is

$$
W F(\mathbf{u})=\left\{(\mathbf{x}, \boldsymbol{\zeta}) \in \mathbb{R}^{6}-\{\mathbf{0}\}: \boldsymbol{\zeta} \in \Sigma_{\mathbf{x}}(\mathbf{u})\right\}
$$

where $\Sigma_{\mathbf{x}}(\mathbf{u})$ is the set of singular frequencies defined as

$$
\Sigma_{\mathbf{x}}(\mathbf{u})=\left\{\boldsymbol{\zeta}:|\widehat{u}(\boldsymbol{\zeta})| \leqslant C_{k_{0}}(1+|\boldsymbol{\zeta}|)^{-k_{0}}, \forall k_{0}\right\}^{c}
$$

Bicharacteristic strip is a key notion to derive TBC/ABC. For $P$ belonging to $O P S^{m}$, its leading symbol $p_{m}(\mathbf{x}, \boldsymbol{\zeta})$, has a degree of homogeneity equal to $m$ and the bicharacteristic strips are integral curves of the following Hamilton-Jacobi equations

$$
\partial_{s} \mathbf{x}=\nabla_{\zeta} p_{m}, \quad \partial_{s} \boldsymbol{\zeta}=-\nabla_{\mathbf{x}} p_{m}
$$


First, it is easy to see that the solution to (1) propagates along bicharacteristic strips, whose union is equal to the wavefront of the solution. In addition if $\left(\mathbf{x}_{0}, \boldsymbol{\zeta}_{0}\right)=\left(x_{0}, y_{0}, t_{0}, \xi_{0}, \eta_{0}, \tau_{0}\right)$ belongs to $W F(\mathbf{u})$ so does $(\mathbf{x}, \boldsymbol{\zeta})=(x(s), y(s), t(s), \xi(s), \eta(s), \tau(s))($ for $s \geqslant 0)$. If $p_{m}\left(\mathbf{x}_{0}, \zeta_{0}\right)=$ 0 , such strips are called the null-bicharacteristic strips. The wavefront of the solution is the union of all the bicharacteristic curves emanating from points where the principal symbol is zero. For the derivation of $\mathrm{ABC}$, a fundamental question will be the identification of points $\left(\mathbf{x}_{0}, \zeta_{0}\right)$ of the cotangent bundle of the domain boundary, which belong to the wavefront (see below). From there, outgoing/incoming bicharacteristic strips will be identified, and $P$ will be factorized into pseudo-differential operators, according to outgoing or incoming strips. We also recall that $W F(\mathbf{u})$ is included in the hyperbolic region of $P$, that is the region where $\operatorname{det}\left(p_{m}\right)$ has real roots. These roots belong to $W F(\mathbf{u})$ and, as recalled above, the corresponding null-bicharacteristic strips will remain in $W F(\mathbf{u})$. A general algorithm to derive $\mathrm{TBC} / \mathrm{ABC}$ consists of

- Determining the symbol of Operator $P$.

- Determining the roots of the leading symbol $\operatorname{det}\left(p_{m}\right)$.

- Constructing the bicharacteristic strips and selecting outgoing ones.

- Factorizing $P$ as product of operators to specify the outgoing wave field.

The factorization of $P$ is in principle possible thanks to theorems (which is recalled in a general framework) such as [1].

Theorem 1.1. Let us consider $P \in O P S^{m}$ a differential operator of the form $\sum_{k+l \leqslant m} a_{k, l} D_{x}^{k} D_{t}^{l}$. Then, there exists a factorization of the form

$$
P=\left(D_{x}-\lambda_{1}(x, t) D_{t}\right) \cdots\left(D_{x}-\lambda_{m}(x, t) D_{t}\right)+\operatorname{order}(m-1) \text { terms },
$$

where $\lambda_{j} \xi$ denotes the distinct real roots (in the hyperbolic region) in $\tau$ of the principal symbol $p_{m}=\sum_{k+l \leqslant m} a_{k, l} \xi^{k} \tau^{l}$. Here, we have set: $D_{x, t}:=-i \partial_{x, t}$.

Geometrical Transformation. We now specify a little more the mixed problem that we will consider. We first introduce a bounded domain $\Omega \subseteq \mathbb{R}^{2}$, which will be assumed to be convex, with smooth fictitious boundary $\Gamma$, and such that it strictly contains $\operatorname{supp}\left(\mathbf{u}_{0}\right)$. We denote $\Omega_{T}:=\Omega \times[0, T]$. We search for $\mathcal{B}$ such that the solution $\tilde{\mathbf{u}}$ to the following mixed problem

$$
\begin{cases}P\left(x, y, t, \partial_{t}, \partial_{x}, \partial_{y}\right) \tilde{\mathbf{u}}(x, y, t)=\mathbf{0}, & \text { on } \Omega_{T} \\ \mathcal{B} \tilde{\mathbf{u}}=\mathbf{0}, & \text { on } \Gamma \times[0, T] \\ \tilde{\mathbf{u}}(x, y, 0)=\mathbf{u}_{0}(x, y), & \text { on } \Omega,\end{cases}
$$

in the appropriate Hilbert space $H$, is such that $\left\|\mathbf{u}_{\mid \Omega}-\tilde{\mathbf{u}}\right\|_{H}$ is as small as possible. Roughly speaking, when the error is zero, the BC are said to be transparent (TBC), and absorbing if not (ABC). For TDDE and TDKGE, we will search for $\mathcal{B}$ in the form

$$
\mathcal{B} \mathbf{u}=\left(\frac{\partial}{\partial \mathbf{n}}+\Lambda^{+}\right) \mathbf{u}=\mathbf{0} \text { on } \Gamma .
$$


For any $\left(x_{0}, y_{0}\right) \in \Gamma$ and $\mathbf{n}\left(\left(x_{0}, y_{0}\right)\right)$ normal vector to $\Gamma$ at $\left(x_{0}, y_{0}\right)$, we study the outgoing and incoming (reflected) bicharacteristic strips. Although the approach which is used here is applicable to any domain with smooth boundary, it is natural (if possible) to choose a fictitious domain $\Omega$ with simple geometry. In this paper we will consider circular domains $\Omega$ of radius $R$ and center $(0,0)$. In that case the curvature is constant which simplifies a lot the algebraic, analytical and numerical computations. We then introduce locally on the boundary a system of polar coordinates which are denoted by $(r, \theta)$ with co-variables $(\rho, \omega)$, and $\left(\mathbf{e}_{r}, \mathbf{e}_{\theta}\right)$ are the normal and angular directions at the boundary $\Gamma$. In particular, any point $(r=0, \theta)$ belongs to the boundary. We also consider the annular region $\Gamma_{r}=$ $\{(r, \theta), r \in[0, \varepsilon)\}$. We now introduce the cotangent bundle to $\Sigma_{T}=\Gamma \times[0, T]$, denoted by $T^{*}\left(\Sigma_{T}\right)$. Its hyperbolic region, in which the solution will propagate, is defined by

$\mathcal{H}\left(\Sigma_{T}\right)=\left\{\left(\theta_{0}, t_{0}, \omega_{0}, \tau_{0}\right) \in T^{*}\left(\Sigma_{T}\right): \operatorname{det}\left(p_{m}\left(0, \theta_{0}, t_{0}, \rho_{0}, \omega_{0}, \tau_{0}\right)\right)=0\right.$ has real roots in $\left.\rho_{0}\right\}$.

Naturally the elliptic region (where the symbol does not vanish) does not contain any relevant information as (" $p \widehat{u}=0$ "), and the glancing region is reduced to $\left(\theta_{0}, t_{0}, \xi_{0}, t_{0}\right)$ due to geometrical assumptions on $\Omega$.

The Hamiltonian equations with initial condition $\left(0, \theta_{0}, t_{0}, \rho_{0}^{ \pm}, \omega_{0}, \tau_{0}\right)$ write

$$
\left\{\begin{array}{lll}
r^{\prime}(s)=\frac{\partial p_{m}}{\partial \rho}, & \theta^{\prime}(s)=\frac{\partial p_{m}}{\partial \omega}, & t^{\prime}(s)=\frac{\partial p_{m}}{\partial \tau}, \\
\rho^{\prime}(s)=-\frac{\partial p_{m}}{\partial r}, & \omega^{\prime}(s)=-\frac{\partial p_{m}}{\partial \theta}, & \tau^{\prime}(s)=-\frac{\partial p_{m}}{\partial t} .
\end{array}\right.
$$

Formally, we recall that these equations can be rewritten in the form $H_{p_{m}} \gamma(s)=0$, where $\gamma(s)=(r(s), \theta(s), t(s), \rho(s), \omega(s), \tau(s))$ denotes the bicharacteristic curves and $H_{p_{m}} \in T\left(T^{*}\left(\Sigma_{T}\right)\right)$, which has a basis $\left(\partial_{r}, \partial_{\theta}, \partial_{t}, \partial_{\rho}, \partial_{\omega}, \partial_{\tau}\right)$. We then select the outgoing (resp. incoming) bicharacteristic strips, which corresponds to $r^{\prime}(s)>0$ (resp. $r^{\prime}(s)<0$ ), and we associate to these strips an operator $\Lambda^{+}$(resp. $\Lambda^{-}$). Moreover, $P$ can be formally factorized in the form

$$
P=\left(\frac{\partial}{\partial \mathbf{n}}+\Lambda^{+}\right)\left(\frac{\partial}{\partial \mathbf{n}}-\Lambda^{-}\right)+R, \quad R \in O P S^{-\infty} .
$$

In addition, from Theorem (1.1), $P$ can be rewritten as follows.

$$
P=P_{m}+\text { order }(m-1) \text { terms, }
$$

with

$$
P_{m}:=\left(D_{r}-\lambda_{1}(r, \theta, t) D_{t}\right) \cdots\left(D_{r}-\lambda_{m}(r, \theta, t) D_{t}\right),
$$

where $\lambda_{j} \rho$ denotes real distinct roots, such that, the positive ones, would correspond to the outgoing waves. TBC is then of the form of a Dirichlet-to-Neumann (DtN) operator

$$
\left(\partial_{\mathbf{n}}+\Lambda^{+}\right) \mathbf{u}=\mathbf{0} \text { on } \Gamma,
$$


where $\Lambda^{+}$is associated to the part of $P_{m}$ involving the positive roots only. An instance of this approach will be detailed in the following section. In this goal, asymptotic analysis will be used to analytically determine $\Lambda^{+}$. We need to recall the following (general) definition, see $[1]$

Definition 1.1. Consider $P(\mathbf{x}, D)$ a scalar order $m$ operator, with symbol $p(\mathbf{x}, \boldsymbol{\xi}) \in C^{\infty}$. There exists a sequence $\left\{p_{m-j}(\mathbf{x}, \boldsymbol{\xi})\right\}_{j \in \mathbb{N}}$ such that for $\boldsymbol{\xi} \neq 0$, and any $\boldsymbol{\beta} \in \mathbb{N}^{3}$

$$
D_{\mathbf{x}}^{\boldsymbol{\beta}}\left(p-\sum_{j=0}^{n} p_{m-j}\right)=\mathcal{O}\left(|\boldsymbol{\xi}|^{m-n-1}\right), \forall n \in \mathbb{N}, \text { and when }|\boldsymbol{\xi}| \rightarrow+\infty .
$$

In that case, we note $p(\mathbf{x}, \boldsymbol{\xi}) \sim \sum_{j=0}^{\infty} p_{m-j}(\mathbf{x}, \boldsymbol{\xi})$ and $P\left(\mathbf{x}, \partial_{\mathbf{x}}\right) \sim \sum_{j=0}^{\infty} P_{m-j}\left(\mathbf{x}, \partial_{\mathbf{x}}\right)$, where $P_{j}=$ $O p\left(p_{j}\right)$

Another useful definition is as follows.

Definition 1.2. A symbol $p \in S^{m}$ is said to be classical if $p \sim \sum_{j=0}^{\infty} p_{m-j}$, where the $p_{m-j}$ are homogeneous functions of degree $m-j$, that is for $|\boldsymbol{\xi}| \geqslant 1$ and $\lambda \geqslant 1$,

$$
p_{m-j}(\mathbf{x}, \lambda \boldsymbol{\xi})=\lambda^{m-j} p_{m-j}(\mathbf{x}, \boldsymbol{\xi}) .
$$

For TBC/ABC, we can generally prove that $\Lambda^{+} \in O P S^{m}$ and that its total symbol $\lambda^{+}$ satisfies the following expansion [1]

$$
\lambda^{+} \sim \sum_{j=0}^{\infty} \lambda_{m-j}^{+}
$$

where $\lambda_{m-j}^{+} \in S^{m-j}$ for $j \in \mathbb{N}$ and $\Lambda_{m-j}^{+}:=O p\left(p_{m-j}\right) \in O P S^{m-j}$. Then, $\Lambda^{+}$will be approximated by $O p\left(\sum_{j=0}^{\infty} \lambda_{m-j}^{+}\right)$. The sequence will be determined by the identification of the symbols of the same order in the left- and right-hand sides of (3) assuming $\lambda^{+}$is classical (4).

\subsection{Organization of the paper}

The rest of the paper is organized as follows. In Section 2, we derive ABCs for 1-d and 2-d TDKGE using the technique based on bicharacteristic strips described above. Section 3 is devoted to the construction of ABCs for TDDE using a diagonalization technique derived from several works, mainly $[3,4,5]$. In Section 4, we propose other possible approaches to construct ABCs for TDDE and TDKGE as well as some connections between ABCs for TDKGE and TDDE. Section 5 is dedicated to numerical illustrations. Finally, we conclude in Section 6. 


\section{Absorbing Boundary Conditions for the Klein-Gordon Equation}

\subsection{One-dimensional Case}

\subsubsection{Empirical absorbing conditions}

This short section is devoted to the derivation of simple empirical ABCs. We want here to emphasis the fact that simple options could be provided to limit spurious wave reflections. However, the weakness of this approach is double. First we do not provide any mathematical information on the reflected wave (accuracy, etc) and then the parameters are empirically chosen, not according to a rigorous procedure. The general principle, which is applicable to any kind of wave equation (Schrödinger, wave equation, Maxwell, Klein-Gordon, etc) consists of absorbing the waves that reach the boundary of the computational domain in a buffer zone using a well designed function. We consider the 1-d Klein-Gordon (KG) and Dirac (D) boundary value problems

$$
\begin{cases}P_{K G, D} \psi(x, t) & =0, \quad x \in[-a, a] \\ \mathcal{B}_{K G, D} \psi(x, t) & =0, \quad x \in\{-a, a\} \\ \psi(x, 0) & =\psi_{0}(x) \in \mathbb{C}\left(\text { or } \mathbb{C}^{2}\right)\end{cases}
$$

with

$$
\left\{\begin{array}{l}
P_{K G}=c^{2} \partial_{x}^{2}-\partial_{t}^{2}-\mathrm{i} e V \partial_{t}-\mathrm{i} c^{2} q e A_{x} \partial_{x}-\mathrm{i} e \partial_{t} V-\mathrm{i} c^{2} e \partial_{x} A_{x}+e^{2} V^{2}-c^{2} e^{2} A_{x}^{2}-m^{2} c^{4} \\
P_{D}=\mathrm{i} \partial_{t}-\alpha_{x}\left(-\mathrm{i} c \partial_{x}-e A_{x}(x, t)\right)-\beta m c^{2}+e I_{2} V_{c}(x)
\end{array}\right.
$$

where

$$
\alpha_{x}=\left(\begin{array}{ll}
0 & 1 \\
1 & 0
\end{array}\right), \quad \beta=\left(\begin{array}{cc}
1 & 0 \\
0 & -1
\end{array}\right), \quad I_{2}=\left(\begin{array}{ll}
1 & 0 \\
0 & 1
\end{array}\right)
$$

In the equation, $e$ denotes the particle charge, $m$ its mass and $c$ the speed of light. The electric potential $\left(V, A_{x}\right)$ depends on $(x, t)$. Coulomb potential $V_{c}$ depends on space only. The goal is to provide a boundary operator $\mathcal{B}_{K G, D}$, limiting spurious reflections at the fictitious domain boundary $\{-a, a\}$. Here is the principle of the proposed approach

- Let us denote by $\varepsilon$ and $\gamma$ two positive real numbers, where $\varepsilon \ll a$.

- We solve

$$
\begin{cases}P_{K G, D} \psi(x, t) & =0, \quad x \in[-a, a] \\ \mathcal{B}_{K G, D} \psi(x, t) & =0, \quad x \in\{-a, a\} \\ \psi(x, 0) & =\psi_{0}(x), \quad x \in[-a, a]\end{cases}
$$

- We multiply solution $\psi$ by a function defined for instance, as follows

$$
f(x, t)= \begin{cases}1-e^{-\gamma\left(\frac{x-a}{x-a+\varepsilon}\right)^{2}}, & x \in(a-\varepsilon, a] \\ 1-e^{-\gamma\left(\frac{x+a}{x+a-\varepsilon}\right)^{2}}, & x \in[-a,-a+\varepsilon), \\ 0, & \text { elsewhere }\end{cases}
$$


Naturally $\varepsilon$ and $\gamma$ are two constants empirically chosen that may depend on $A_{x}, V, V_{c}$. However numerical examples show that it can be an easy-to-implement alternative to more rigorous (but also more computationally costly) boundary conditions. Note that this approach is easily extendable to higher dimension, although in that case the empirical choice of parameters is even more problematic (as $\varepsilon, \gamma$ may also depend on the geometry of the fictitious boundary).

\subsubsection{Absorbing boundary conditions for Klein-Gordon's equation in 1-d with a constant laser}

Let us first consider that we are solving the $\mathrm{KG}$ equation for a constant laser, which means that $V$ and $A_{x}$ are both $x$ and $t$ independent. In this case, the KG equation is given by

$$
P_{K G} \varphi=\left(c^{2} \partial_{x}^{2}-\partial_{t}^{2}-\mathrm{i} e V \partial_{t}-\mathrm{i} c^{2} e A_{x} \partial_{x}+e^{2} V^{2}-c^{2} e^{2} A_{x}^{2}-m^{2} c^{4}\right) \varphi(t, x)=0 .
$$

Let us denote by $\tau$ the time Fourier covariable. In this case, Fourier transforming the above equation leads to

$$
\left(c^{2} \partial_{x}^{2}-\mathbf{i} c^{2} e A_{x} \partial_{x}+\left(\tau^{2}+e \tau V+\left(e^{2} V^{2}-c^{2} e^{2} A_{x}^{2}-m^{2} c^{4}\right)\right)\right) \widehat{\varphi}(\tau, x)=0
$$

Computing the roots of this equation yields

$$
\lambda_{1}^{ \pm}(\tau, x)=\frac{\mathrm{i} e A_{x}}{2} \pm \frac{1}{c} \sqrt{-\tau^{2}-e V \tau-e^{2} V^{2}+\frac{3}{4} c^{2} e^{2} A_{x}^{2}+m^{2} c^{4}},
$$

and the solution writes as the superposition of two plane waves

$$
\widehat{\varphi}(\tau, x)=A^{+} e^{\lambda_{1}^{+}(\tau, x) x}+A^{-} e^{\lambda_{1}^{-}(\tau, x) x} .
$$

The left traveling solution is associated with $\lambda_{1}^{-}$(easy to see for the laser free equation). Therefore, to get the outgoing solution, one must have $A^{-}=0$. Deriving according to $\partial_{x}$ provides the following relation

$$
\left(\partial_{x}-\lambda_{1}^{+}\right) \widehat{\varphi}(\tau, x)=0
$$

which shows that the equation selects the outgoing solution to the domain boundary. Going back to the physical domain by inverse Fourier transform, one gets the TBC

$$
\left(c \partial_{x}-\sqrt{\partial_{t}^{2}+\mathrm{i} e V \partial_{t}-e^{2} V^{2}+\frac{3}{4} c^{2} e^{2} A_{x}^{2}+m^{2} c^{4}}-\frac{\mathrm{i} c e A_{x}}{2}\right) \varphi(t, x)=0, \text { on } \Sigma_{T} .
$$

The above DtN operator is nonlocal in time. In the laser free case, one has

$$
\left(c \partial_{x}-\partial_{t}\right) \varphi(t, x)=0
$$

which is just the local TBC for the wave equation part going to the left. 


\subsubsection{Absorbing boundary conditions for Klein-Gordon's equation in 1-d with a variable laser}

The approach which is used to derive ABCs for the Klein-Gordon equation was originally presented in [2], [3], [4] for linear and nonlinear Schrödinger equations. We focus here on the computational details. We recall that the laser-particle Klein-Gordon equation $P_{K G} \varphi=0$, writes

$$
\left[\left(\mathrm{i} \partial_{t}-e V(x, t)\right)^{2}-c^{2}\left(\mathrm{i} \partial_{x}+e A_{x}(x, t)\right)^{2}-m^{2} c^{4}\right] \varphi=0, \quad \text { in }[-a, a]
$$

which we expand into

$$
P_{K G} \varphi=\left[c^{2} \partial_{x}^{2}-\partial_{t}^{2}-\mathrm{i} e V \partial_{t}-\mathrm{i} c^{2} e A_{x} \partial_{x}+O_{D}\right] \varphi=0, \quad \text { in }[-a, a],
$$

where $O_{D}$ is the zeroth-order operator given by

$$
O_{D}:=-\mathbf{i} e \partial_{t} V-\mathbf{i} c^{2} e \partial_{x} A_{x}+e^{2} V^{2}-c^{2} e^{2} A_{x}^{2}-m^{2} c^{4}
$$

We search for an ABC at $x= \pm a$ under the form of approximate DtN operators. The principle of the technique developed in [4] consists of factorizing $P_{K G}$ in two operators as follows

$$
P_{K G}\left(t, x, \partial_{t}, \partial_{x}\right)=\left(c \partial_{x}-\Lambda^{-}\left(t, x, \partial_{t}, \partial_{x}\right)\right)\left(c \partial_{x}+\Lambda^{+}\left(t, x, \partial_{t}, \partial_{x}\right)\right)+R\left(t, x, \partial_{t}, \partial_{x}\right)
$$

where $R\left(t, x, \partial_{t}, \partial_{x}\right)$ is a smoothing operator (in $\left.O P S^{-\infty}\right)$ and $\Lambda^{ \pm}\left(t, x, \partial_{t}, \partial_{x}\right)$ are two pseudodifferential operators to be determined such that $\Lambda^{+}\left(t, x, \partial_{t}, \partial_{x}\right)\left(\operatorname{resp} . \Lambda^{-}\left(t, x, \partial_{t}, \partial_{x}\right)\right)$ has only outgoing (resp. only incoming) bicharacteristic strips at the domain boundary. First, we notice that (omitting $\left(t, x, \partial_{t}, \partial_{x}\right)$ for the sake of conciseness)

$$
\left(c \partial_{x}-\Lambda^{-}\right)\left(c \partial_{x}+\Lambda^{+}\right)=c^{2} \partial_{x}^{2}+c\left(\Lambda^{+}-\Lambda^{-}\right) \partial_{x}+c O p\left(\partial_{x} \lambda^{+}\right)-\Lambda^{-} \Lambda^{+} .
$$

By identification of the operators of different orders in front of $\partial_{x}$, we obtain

$$
c\left(\Lambda^{+}-\Lambda^{-}\right)=-i c^{2} e A_{x}
$$

and

$$
c O p\left(\partial_{x} \lambda^{+}\right)-\Lambda^{-} \Lambda^{+}=-\partial_{t}^{2}-i e V \partial_{t}+O_{D}
$$

Denoting $\lambda^{ \pm}=\sigma\left(\Lambda^{ \pm}\right)$we get at the symbol level

$$
\left\{\begin{array}{l}
c\left(\lambda^{+}-\lambda^{-}\right)=-i c^{2} e A_{x}, \\
c \partial_{x} \lambda^{+}-\lambda^{-} \# \lambda^{+}=\tau^{2}-e V \tau+\sigma\left(O_{D}\right) .
\end{array}\right.
$$

Now according to [28], any classical symbol of $S^{1}$ can be asymptotically expanded as $\lambda^{ \pm} \sim$ $\sum_{j=0}^{+\infty} \lambda_{1-j}^{ \pm}$, where $\lambda_{1-j}^{ \pm}$is the symbol of a pseudodifferential operator of homogeneity degree 
$1-j$, for $j \in \mathbb{N}$. In order to determine the expansion terms it is sufficient to recursively identify the terms of same homogeneity in system (13). For $j=0$, one gets

$$
\left\{\begin{array}{ccc}
\lambda_{1}^{+}-\lambda_{1}^{-} & = & 0 \\
-\lambda_{1}^{-} \lambda_{1}^{+} & = & \tau^{2}
\end{array} .\right.
$$

For the next homogeneity, we obtain for $j=1$

$$
\left\{\begin{array}{l}
c\left(\lambda_{0}^{+}-\lambda_{0}^{-}\right)=-i c^{2} e A_{x} \\
\partial_{x} \lambda_{1}^{+}-\lambda_{1}^{-} \lambda_{0}^{+}-\lambda_{0}^{-} \lambda_{1}^{+}+i \partial_{\xi, \tau} \lambda_{1}^{-} \partial_{x, t} \lambda_{1}^{+}=-e V \tau .
\end{array}\right.
$$

For $j=2$, we have the following expression

$$
\left\{\begin{array}{l}
c\left(\lambda_{-1}^{+}-\lambda_{-1}^{-}\right)=0 \\
\partial_{x} \lambda_{0}^{+}-\lambda_{1}^{-} \lambda_{-1}^{+}-\lambda_{0}^{-} \lambda_{0}^{+}-\lambda_{-1}^{-} \lambda_{1}^{+} \\
+i \partial_{\xi, \tau} \lambda_{1}^{-} \partial_{x, t} \lambda_{0}^{+}+i \partial_{\xi, \tau} \lambda_{0}^{-} \partial_{x, t} \lambda_{1}^{+}+\frac{1}{2} \partial_{\xi, \tau}^{2} \lambda_{1}^{-} \partial_{x, t}^{2} \lambda_{1}^{+}=\sigma\left(O_{D}\right)
\end{array}\right.
$$

and finally for $j=3$

$$
\left\{\begin{array}{l}
c\left(\lambda_{-2}^{+}-\lambda_{-2}^{-}\right)=0 \\
\partial_{x} \lambda_{-1}^{+}-\lambda_{1}^{-} \lambda_{-2}^{+}-\lambda_{0}^{-} \lambda_{-1}^{+}-\lambda_{-1}^{-} \lambda_{0}^{+}-\lambda_{-2}^{-} \lambda_{1}^{+} \\
+i \partial_{\xi, \tau} \lambda_{1}^{-} \partial_{x, t} \lambda_{-1}^{+}+i \partial_{\xi, \tau} \lambda_{0}^{-} \partial_{x, t} \lambda_{0}^{+}+i \partial_{\xi, \tau} \lambda_{-1}^{-} \partial_{x, t} \lambda_{1}^{+} \\
+\frac{1}{2} \partial_{\xi, \tau}^{2} \lambda_{1}^{-} \partial_{x, t}^{2} \lambda_{0}^{+}+\frac{1}{2} \partial_{\xi, \tau}^{2} \lambda_{0}^{-} \partial_{x, t}^{2} \lambda_{1}^{+}-\frac{i}{6} \partial_{\xi, \tau}^{3} \lambda_{1}^{-} \partial_{x, t}^{3} \lambda_{1}^{+}=0
\end{array}\right.
$$

By using system (14), we have

$$
\left\{\begin{array}{l}
\lambda_{1}^{+}=\lambda_{1}^{-}, \\
\lambda_{1}^{+}= \pm\left(-\tau^{2}\right)^{1 / 2}= \pm \mathrm{i} \tau .
\end{array}\right.
$$

At this point, we have to select the forward characteristic, which corresponds to the one that satisfies $x^{\prime}(s)>0$ at $x=+a\left(\right.$ resp. $x^{\prime}(s)<0$ at $\left.x=-a\right)$, where

$$
\begin{aligned}
& x^{\prime}(s)=\partial_{\xi} p_{2}, \quad t^{\prime}(s)=\partial_{\tau} p_{2}, \\
& \xi^{\prime}(s)=-\partial_{x} p_{2}, \quad \tau^{\prime}(s)=-\partial_{t} p_{2},
\end{aligned}
$$

with principal symbol $p_{2}(x, t, \xi, \tau)=-c^{2} \xi^{2}+\tau^{2}$ and for $\left( \pm a, t_{0}, \xi_{0}, \tau_{0}\right)$ such that $p_{2}\left(x_{0}, t_{0}, \xi_{0}, \tau_{0}\right)=$ 0 . We get the following roots $\xi= \pm \tau / c$ in the hyperbolic region of the operator (corresponding to propagating waves). Now as $x^{\prime}(s)=-2 c^{2} \xi$, we conclude that the forward characteristic is then the one associated to $-\tau / c$ at $x=+a$ (and $+\tau / c$ for $x=-a$ ), that is $\lambda_{1}^{+}=\mathrm{i} \tau$. Fixing the principal symbols $\lambda_{1}^{ \pm}$(as previously) defines uniquely the asymptotic expansion of $\lambda^{ \pm}$and also the classical first-order pseudodifferential operators $\Lambda^{ \pm}$. For building an accurate boundary condition, we compute the next three terms of the symbolical asymptotic expansion. This leads to

$$
\left\{\begin{array}{l}
\lambda_{0}^{+}=\frac{i}{2}\left(e V-c e A_{x}\right), \\
\lambda_{-1}^{+}=\frac{i}{2}\left[\sigma\left(O_{D}\right)-\frac{i}{2} \sigma\left(O_{G_{x}}\right)+\frac{i}{2} \sigma\left(O_{G_{t}}\right)-\frac{1}{4} \sigma\left(O_{F}\right)\right] \tau^{-1}, \\
\lambda_{-2}^{+}=\lambda_{-2}^{+}=\frac{1}{4}\left[\partial_{x}-\partial_{t}-i e V\right]\left[\sigma\left(O_{D}\right)-\frac{i}{2} \sigma\left(O_{G_{x}}\right)+\frac{i}{2} \sigma\left(O_{G_{t}}\right)-\frac{1}{4} \sigma\left(O_{F}\right)\right] \tau^{-2},
\end{array}\right.
$$


where the operators $O_{F}, O_{G_{x}}$ and $O_{G_{t}}$ are defined as follows

$$
\left\{\begin{aligned}
O_{G_{x}} & :=e \partial_{x} V-c e \partial_{x} A_{x} \\
O_{G_{t}} & :=e \partial_{t} V-c e \partial_{t} A_{x} \\
O_{F} & :=e^{2} V^{2}-c^{2} e^{2} A_{x}^{2}
\end{aligned}\right.
$$

Let us now provide the following factorization result which is at the basis of constructing ABCs of arbitrary order for the general situation. We do not give the details of the proof which can be obtained by adapting the results in [].

Proposition 2.1. There exists two unique first-order pseudodifferential operators $\Lambda_{k}^{+}$and $\Lambda_{k}^{-}$such that

$$
P_{K G}=\left(c \partial_{x}+\Lambda_{k}^{+}\right)\left(c \partial_{x}-\Lambda_{k}^{-}\right)+R_{2-k}
$$

where $R_{2-k} \in O P S^{2-k}$ and $\Lambda_{k}^{+}=O p\left(\sigma_{k}^{+}\right)$, with $\sum_{j=0}^{k-1} \lambda_{1-j}^{+}$. Here, we have fixed $\lambda_{1}^{+}:=\mathrm{i} \tau$ and the $\lambda_{1-j}^{+}$are defined in (18) for $j=1,2,3$.

By truncating the formal expansion $\sum_{j=0}^{\infty} \lambda_{1-j}^{+}$up to the $k$ first terms, a $k$-th order ABC is obtained through the boundary condition

$$
\left(c \partial_{\mathbf{n}}+\Lambda_{k}^{+}\right) \varphi=0, \text { on } \Sigma_{T} .
$$

We recall that the Riemann-Liouville operator is defined as follows (for $\nu>0$ )

$$
D^{-\nu} f(t)=\frac{1}{\Gamma(\nu)} \int_{0}^{t}(t-s)^{(\nu-1)} f(s) d s
$$

and has a symbol which is equal to $(\mathrm{i} \tau)^{-\nu}$. When $A_{x}=V=0$, which is the laser-free TDKGE, we easily see that

$$
\sigma_{3}^{+}=\sum_{j=0}^{1} \lambda_{1-j}^{+}=\mathbf{i} \tau+\frac{m^{2} c^{4}}{2 i \tau}
$$

so that

$$
\Lambda_{2}^{+}=\partial_{t}+\frac{m^{2} c^{4}}{2} \partial_{t}^{-1}
$$

More generally, for $A_{x} \neq 0, V \neq 0$, we have

$$
\left\{\begin{array}{l}
\Lambda_{1}^{+}=\partial_{t}, \\
\Lambda_{2}^{+}=\partial_{t}+\frac{i e V}{2}-\frac{i c e A_{x}}{2}, \\
\Lambda_{3}^{+}=\partial_{t}+\frac{i e V}{2}-\frac{i c e A_{x}}{2}-\frac{1}{2}\left(O_{D}-\frac{1}{4} O_{F}-\frac{i}{2} O_{G_{x}}+\frac{i}{2} O_{G_{t}}\right) \partial_{t}^{-1}
\end{array}\right.
$$

where $O_{D}, O_{F}, O_{G}$ are defined in $(12),(19)$. 


\subsection{Two-dimensional TDKE}

\subsubsection{Laser-free TDKGE in 2-d}

We consider a circular domain of radius $R$, on which we plan to provide an ABC. In that case, it is natural to consider polar coordinates in all $\Omega$ and conditions have to be imposed at $r=R$. Another possibility is to keep cartesian coordinates and to search for local coordinates on the circle. In fine, the treatment at the boundary will be the same. However the second approach is more general as it is applicable, in principle, to any smooth boundary, whatever the system of coordinates used inside the domain. For $r \in[0, \varepsilon)$ with $\varepsilon>0$ small enough and denoting by $\chi_{r}=(R+r)$ the radius of the circular domain $\Omega_{r}$ with boundary $\Gamma_{r}:=\partial \Omega_{r}$, the TDKGE in local polar coordinates in $\Sigma_{r}=\Gamma_{r} \times[0, T]$ writes,

$$
P_{K G}^{(r, \theta)} \varphi=\left[-\partial_{t}^{2}+c^{2} \partial_{r}^{2}-c^{2} \frac{1}{\chi_{r}} \partial_{r}+c^{2} \frac{1}{\chi_{r}^{2}} \partial_{\theta}^{2}-m^{2} c^{4}\right] \varphi(t, r, \theta)=0,
$$

for $(r, \theta) \in \Gamma_{r}$, and $t \in \mathbb{R}^{+}$. Variables $(r, \theta, t)$ have duals denoted by $(\rho, \xi, \tau)$. The principal symbol of $P_{K G}^{(r, \theta)}$ is given by

$$
p_{2}(r, \theta, t, \rho, \xi, \tau)=\tau^{2}-c^{2} \rho^{2}-\frac{c^{2}}{\chi_{r}^{2}} \xi^{2} .
$$

We are first interested in the bicharacteristic strips at the domain boundary (where $r=0$ ). We have to select a point, in the hyperbolic region of this operator, that will belong to the wavefront of the solution,

$$
p_{2}\left(0, \theta_{0}, t_{0}, \tau_{0}, \rho_{0}, \xi_{0}\right)=\tau_{0}^{2}-c^{2} \rho_{0}^{2}-\frac{c^{2}}{\chi_{r}^{2}} \xi_{0}^{2}=0 .
$$

The solutions are $\rho_{0}^{ \pm}= \pm\left(\frac{\tau_{0}^{2}}{c^{2}}-\frac{\xi_{0}^{2}}{\chi_{r}^{2}}\right)^{1 / 2}$. The hyperbolic region of the cotangent bundle $T^{*}\left(\Sigma_{r}\right)$ is the region where $\tau_{0}^{2} / c^{2}-\xi_{0}^{2} / \chi_{r}^{2}>0$ (then $p_{m}$ has real roots). In the elliptic region the waves are evanescent. The Hamilton-Jacobi equations write

$$
\begin{cases}r^{\prime}(s)=\partial_{\rho} p_{m}, & \rho^{\prime}(s)=-\partial_{r} p_{m} \\ \theta^{\prime}(s)=\partial_{\xi} p_{m}, & \xi^{\prime}(s)=-\partial_{\theta} p_{m} \\ t^{\prime}(s)=\partial_{\tau} p_{m}, & \tau^{\prime}(s)=-\partial_{t} p_{m}\end{cases}
$$

The outgoing characteristics are defined by the condition $r^{\prime}(s)>0$. Since $r^{\prime}(s)=\partial_{\rho} p_{m}=$ $-2 \rho$, we prove that the outgoing characteristic corresponds to

$$
\rho^{-}=-\left(\frac{\tau^{2}}{c^{2}}-\frac{\xi^{2}}{\chi_{r}^{2}}\right)^{1 / 2} .
$$

To build the $\mathrm{ABC}$, we now consider a factorization of the operator $P_{K G}^{(r, \theta)}$ as

$$
P_{K G}^{(r, \theta)}\left(r, \theta, t, \partial_{t}, \partial_{r}, \partial_{\theta}\right)=\left(c \partial_{r}-\Lambda^{-}\left(r, \theta, t, \partial_{\theta}, \partial_{t}\right)\right)\left(c \partial_{r}+\Lambda^{+}\left(r, \theta, t, \partial_{\theta}, \partial_{t}\right)\right)+R
$$


where $\Lambda^{ \pm} \in O P S^{1}$ has a total symbol $\lambda^{ \pm}$and a principal symbol equal to $\rho^{ \pm}$, respectively, and $R \in O P S^{\infty}$. We now have to explicitly compute $\Lambda^{ \pm}$. Since we have

$$
P_{K G}^{(r, \theta)} \sim\left(c \partial_{r}-\Lambda^{-}\right)\left(c \partial_{r}+\Lambda^{+}\right)=c^{2} \partial_{r}^{2}+c\left(\Lambda^{+}-\Lambda^{-}\right) \partial_{r}+c O p\left(\partial_{r} \lambda^{+}\right)-\Lambda^{-} \Lambda^{+},
$$

the expression $(22)$ of $P_{K G}^{(r, \theta)}$ leads to the following system by identification

$$
\left\{\begin{array}{l}
c\left(\lambda^{+}-\lambda^{-}\right)=-\frac{c^{2}}{\chi_{r}} \\
c \partial_{r} \lambda^{+}-\lambda^{-} \# \lambda^{+}=\tau^{2}-\frac{c^{2}}{\chi_{r}^{2}} \xi^{2}-m^{2} c^{4} .
\end{array}\right.
$$

As an element of $S^{1}, \lambda^{ \pm}$can be expanded as $\lambda^{ \pm} \sim \sum_{j=0}^{\infty} \lambda_{1-j}^{ \pm}$. Furthermore, let us recall

We also recall that, denoting $\lambda^{-} \# \lambda^{+}=\sigma\left(\Lambda^{-} \Lambda^{+}\right)$,

$$
\begin{aligned}
\sigma\left(\Lambda^{-} \Lambda^{+}\right)=\lambda^{-} \# \lambda^{+}= & \left(\sum_{j=0}^{\infty} \lambda_{1-j}^{-}\right)\left(\sum_{j=0}^{\infty} \lambda_{1-j}^{+}\right)-i\left(\sum_{j=0}^{\infty} \partial_{\rho, \xi, \tau} \lambda_{1-j}^{-}\right)\left(\sum_{j=0}^{\infty} \partial_{r, \theta, t} \lambda_{1-j}^{+}\right) \\
& -\frac{1}{2}\left(\sum_{j=0}^{\infty} \partial_{\rho, \xi, \tau}^{2} \lambda_{1-j}^{-}\right)\left(\sum_{j=0}^{\infty} \partial_{r, \theta, t}^{2} \lambda_{1-j}^{+}\right)+\sum_{\alpha \geqslant 3} \frac{(-i)^{\alpha}}{\alpha !} \partial_{\rho, \xi, \tau}^{\alpha} \lambda_{1-j}^{-} \partial_{r, \theta, t}^{\alpha} \lambda_{1-j}^{+} .
\end{aligned}
$$

In the following, we will use the notation

$$
\sigma_{\alpha}\left(\Lambda^{-} \Lambda^{+}\right)=\frac{(-i)^{\alpha}}{\alpha !} \partial_{\rho, \xi, \tau}^{\alpha} \lambda^{-} \partial_{r, \theta, t}^{\alpha} \lambda^{+}
$$

As before, we consider symbols up to those in $S^{-2}$ that is, we approximate $\lambda^{ \pm}$by $\sum_{j=0}^{3} \lambda_{1-j}^{ \pm}$, and we identify terms of the same homogeneity order. This leads in $\lambda^{-} \# \lambda^{+}$to

- Order 2: $\lambda_{1}^{-} \lambda_{1}^{+}$.

- Order 1: $\lambda_{1}^{-} \lambda_{0}^{+}+\lambda_{0}^{-} \lambda_{1}^{+}-\mathrm{i} \partial_{\rho, \xi, \tau} \lambda_{1}^{-} \partial_{r, \theta, t} \lambda_{1}^{+}$.

- Order 0: $\lambda_{-1}^{-} \lambda_{1}^{+}+\lambda_{0}^{-} \lambda_{0}^{+}+\lambda_{1}^{-} \lambda_{-1}^{+}-\mathrm{i} \partial_{\rho, \xi, \tau} \lambda_{1}^{-} \partial_{r, \theta, t} \lambda_{0}^{+}-\mathrm{i} \partial_{\rho, \xi, \tau} \lambda_{0}^{-} \partial_{r, \theta, t} \lambda_{1}^{+}-\frac{1}{2} \partial_{\rho, \xi, \tau}^{2} \lambda_{1}^{-} \partial_{r, \theta, t}^{2} \lambda_{1}^{+}$

- Order -1: $\lambda_{-2}^{-} \lambda_{1}^{+}+\lambda_{-1}^{-} \lambda_{0}^{+}+\lambda_{0}^{-} \lambda_{-1}^{+}+\lambda_{1}^{-} \lambda_{-2}^{+}-i \partial_{\rho, \xi, \tau} \lambda_{1}^{-} \partial_{r, \theta, t} \lambda_{-1}^{+}-i \partial_{\rho, \xi, \tau} \lambda_{0}^{-} \partial_{r, \theta, t} \lambda_{0}^{+}$

$$
-\mathrm{i} \partial_{\rho, \xi, \tau} \lambda_{-1}^{-} \partial_{r, \theta, t} \lambda_{1}^{+}-\frac{1}{2} \partial_{\rho, \xi, \tau}^{2} \lambda_{1}^{-} \partial_{r, \theta, t}^{2} \lambda_{0}^{+}-\frac{1}{2} \partial_{\rho, \xi, \tau}^{2} \lambda_{0}^{-} \partial_{r, \theta, t}^{2} \lambda_{1}^{+}+\frac{i}{6} \partial_{\rho, \xi, \tau}^{3} \lambda_{1}^{-} \partial_{r, \theta, t}^{3} \lambda_{1}^{+} \text {. }
$$

In fact, in the laser-free configuration, as $\lambda_{1-j}^{ \pm}$only depends on $r, \tau, \xi$, we easily verify that $\sigma_{\alpha}\left(\Lambda^{-} \Lambda^{+}\right)=0$ for $\alpha \neq 0$. We deduce

$$
\left\{\begin{array}{l}
c\left(\lambda_{1}^{+}-\lambda_{1}^{-}\right)=0 \\
-\lambda_{1}^{-} \lambda_{1}^{+}=\tau^{2}-\frac{c^{2}}{\chi_{r}^{2}} \xi^{2}
\end{array}\right.
$$

we get up to leading order:

$$
\left\{\begin{array}{l}
\lambda_{1}^{+}=\lambda_{1}^{-} \\
\lambda_{1}^{+}= \pm\left(-\tau^{2}+\frac{c^{2}}{\chi_{r}^{2}} \xi^{2}\right)^{1 / 2}
\end{array}\right.
$$


As we select the forward bicharacteristics

$$
\lambda_{1}^{+}=\left(-\tau^{2}+\frac{c^{2}}{\chi_{r}^{2}} \xi^{2}\right)^{1 / 2},
$$

Then

$$
\begin{cases}c\left(\lambda_{0}^{+}-\lambda_{0}^{-}\right) & =-\frac{c^{2}}{\chi_{r}} \\ c \partial_{r} \lambda_{1}^{+}-\lambda_{1}^{-} \lambda_{0}^{+}-\lambda_{0}^{-} \lambda_{1}^{+} & =0\end{cases}
$$

so that

$$
\begin{cases}\lambda_{0}^{-} & =\lambda_{0}^{+}+\frac{c}{\chi_{r}} \\ -\lambda_{1}^{+}\left(2 \lambda_{0}^{+}+\frac{c}{\chi_{r}}\right) & =-c \partial_{r} \lambda_{1}^{+}\end{cases}
$$

and

$$
\left\{\begin{array}{l}
\lambda_{0}^{+}=\frac{c}{2 \lambda_{1}^{+}}\left(\partial_{r} \lambda_{1}^{+}-\frac{c}{\chi_{r}}\right) \\
c \partial_{r} \lambda_{1}^{+}=c \partial_{r}\left(-\tau^{2}+\frac{c^{2}}{\chi_{r}^{2}} \xi^{2}\right)^{1 / 2}
\end{array}\right.
$$

Considering the next order

$$
\begin{aligned}
& \begin{cases}c\left(\lambda_{-1}^{+}-\lambda_{-1}^{-}\right) & =0 \\
c \partial_{r} \lambda_{0}^{+}-\lambda_{1}^{-} \lambda_{-1}^{+}-\lambda_{0}^{-} \lambda_{0}^{+}-\lambda_{-1}^{-} \lambda_{1}^{+} & =-m^{2} c^{4}\end{cases} \\
& \begin{cases}c\left(\lambda_{-2}^{+}-\lambda_{-2}^{-}\right) & =0 \\
c \partial_{r} \lambda_{-1}^{+}-\lambda_{1}^{-} \lambda_{-2}^{+}-\lambda_{0}^{-} \lambda_{-1}^{+}-\lambda_{-1}^{-} \lambda_{0}^{+}-\lambda_{-2}^{-} \lambda_{1}^{+} & =0\end{cases}
\end{aligned}
$$

and for $\lambda_{-1}^{+}$we get

$$
\left\{\begin{array}{l}
\lambda_{-1}^{+}=\lambda_{-1}^{+} \\
\lambda_{-1}^{-}=\frac{1}{2 \lambda_{1}^{+}}\left(m^{2} c^{4}+c \partial_{r} \lambda_{0}^{+}-\lambda_{0}^{-} \lambda_{0}^{+}\right)
\end{array}\right.
$$

and finally $\lambda_{-2}^{+}$gives us

$$
\left\{\begin{array}{l}
\lambda_{-2}^{+}=\lambda_{-2}^{+} \\
\lambda_{-2}^{-}=\frac{1}{2 \lambda_{1}^{+}}\left(-\lambda_{-1}^{+}\left(2 \lambda_{0}^{+}+\frac{c}{\chi_{r}}\right)+c \partial_{r} \lambda_{-1}^{+}\right) .
\end{array}\right.
$$


Thus

$$
\begin{aligned}
\sum_{j=0}^{3} \lambda_{1-j}^{+} & =\left(-\tau^{2}+\frac{c^{2}}{\chi_{r}^{2}} \xi^{2}\right)^{1 / 2}+\frac{c}{2 \lambda_{1}^{+}}\left(\partial_{r} \lambda_{1}^{+}-\frac{c}{\chi_{r}}\right)+\frac{1}{2 \lambda_{1}^{+}}\left[m^{2} c^{4}+c \partial_{r} \lambda_{0}^{+}-\lambda_{0}^{-} \lambda_{0}^{+}\right] \\
& =\frac{1}{2 \lambda_{1}^{+}}\left(-\lambda_{-1}^{+}\left(2 \lambda_{0}^{+}+\frac{c}{\chi_{r}}\right)+c \partial_{r} \lambda_{-1}^{+}\right) .
\end{aligned}
$$

We denote $\sum_{j=-1}^{3} \lambda_{1-j}^{+}$by $\lambda_{(-2,1)}^{+}$and $\Lambda_{(-2,1)}^{+}=O p\left(\lambda_{(-2,1)}^{+}\right)$. Thus

Proposition 2.2. There exist 2 operators $\Lambda_{(-2,1)}^{+}, \Lambda_{(-2,1)}^{-}$such that

$$
P_{K G}^{r, \theta}=\left(c \partial_{x}+\Lambda_{(-2,1)}^{+}\right)\left(c \partial_{x}-\Lambda_{(-2,1)}^{-}\right)+R_{-3}
$$

where $R_{-3} \in O P S^{-3}$. And as a consequence, the TBC

$$
\left[c \partial_{r}+\Lambda^{+}\right] \varphi=0
$$

is approximated by the following $A B C$ :

$$
\left[c \partial_{r}+\Lambda_{(-2,1)}^{+}\right] \varphi=0 .
$$

In the next chapter we will explicitely define the pseudo-differential operators appearing in (23).

\subsubsection{Laser-particle TDKGE in 2-d}

We now consider the full TDKGE including the electromagnetic field: $\mathbf{A}(x, y, t)$ and $V(x, y, t)$ which represent the vectorial and scalar potentials satisfying Maxwell's equations. They are assumed given and regular enough. As before, we fix $\hbar=1$. The equation writes

$$
\left[\left(\mathrm{i} \partial_{t}-e V(x, y, t)\right)^{2}-c^{2}\left(\frac{1}{\mathrm{i}} \nabla-e \mathbf{A}(x, y, t)\right)^{2}-m^{2} c^{4}\right] \varphi=0 .
$$

Expanding the equation leads to

$$
\left[-\partial_{t}^{2}+c^{2} \Delta-\mathbf{i} e V \partial_{t}-i c^{2} e \mathbf{A} \cdot \nabla-\mathbf{i} e \partial_{t} V-i c^{2} e \nabla \cdot \mathbf{A}+e^{2} V^{2}-c^{2} e^{2} \mathbf{A}^{2}-m^{2} c^{4}\right] \varphi=0 .
$$

The domain $\Omega$ is again a disk of radius $R$. We then need to rewrite in $\Sigma_{r}$, for $r \in[0, \varepsilon)$ with $\varepsilon>0$ small enough, the TDKGE in polar coordinate, that is (details are skipped)

$$
\begin{aligned}
P_{K G L}^{r, \theta} \varphi= & {\left[-\partial_{t}^{2}+c^{2} \partial_{r}^{2}-\frac{c^{2}}{\chi_{r}} \partial_{r}+\frac{c^{2}}{\chi_{r}^{2}} \partial_{\theta}^{2}-i e V \partial_{t}-i c^{2} e A_{r} \partial_{r}-i \frac{c^{2}}{\chi_{r}} e A_{\theta} \partial_{\theta}\right.} \\
& \left.-i e \partial_{t} V-i c^{2} e \partial_{r} A_{r}-i \frac{c^{2}}{\chi_{r}} e A_{r}-\frac{i c^{2} e}{\chi_{r}} \partial_{\theta} A_{\theta}-c^{2} e^{2} A_{r}^{2}-c^{2} e^{2} A_{\theta}^{2}+e^{2} V^{2}-m^{2} c^{4}\right],
\end{aligned}
$$

where

$$
\left\{\begin{array}{l}
A_{x}=A_{r} \cos (\theta)-A_{\theta} \sin (\theta) \\
A_{y}=A_{r} \sin (\theta)+A_{\theta} \cos (\theta)
\end{array}\right.
$$


Some notations are introduced. First the following operators :

$$
\left\{\begin{aligned}
O_{B} & :=-\left(\frac{c^{2}}{\chi_{r}}+c^{2} \mu A_{r}\right) \partial_{r} \\
O_{F} & :=-\frac{c^{2} \mu}{\chi_{r}} A_{\theta} \partial_{\theta} \\
O_{D} & :=-\mu \partial_{t} V-c^{2} \mu \partial_{r} A_{r}-\frac{c^{2} \mu}{\chi_{r}} A_{r}-\frac{c^{2} \mu}{\chi_{r}} \partial_{\theta} A_{\theta}+e^{2} V^{2}-c^{2} e^{2} \mathbf{A}^{2}-m^{2} c^{4}
\end{aligned}\right.
$$

and the functions

$$
\left\{\begin{aligned}
\mu & :=i e \\
q_{O_{B}} & :=-\frac{c^{2}}{\chi_{r}}-c^{2} \mu A_{r} \\
q_{O_{F}} & :=-\frac{c^{2} \mu}{\chi_{r}} A_{\theta} \\
q_{O_{D}} & :=-\mu \partial_{t} V-c^{2} \mu \partial_{r} A_{r}-\frac{c^{2} \mu}{\chi_{r}} A_{r}-\frac{c^{2} \mu}{\chi_{r}} \partial_{\theta} A_{\theta}+e^{2} V^{2}-c^{2} e^{2} \mathbf{A}^{2}-m^{2} c^{4}
\end{aligned}\right.
$$

This allows us to rewrite the equation in a more compact form

$$
\left(c^{2} \partial_{r}^{2}-\partial_{t}^{2}+\frac{c^{2}}{\chi_{r}^{2}} \partial_{\theta}^{2}-\mu V \partial_{t}+O_{B}+O_{F}+O_{D}\right) \varphi=0
$$

As before we plan to factorize this operator $P_{K G}^{(r, \theta)}$ in the form $\left(c \partial_{r}-\Lambda^{-}\right)\left(c \partial_{r}+\Lambda^{+}\right)$. We recall that

$$
\left(c \partial_{r}-\Lambda^{-}\right)\left(c \partial_{r}+\Lambda^{+}\right)=c^{2} \partial_{r}^{2}+c\left(\Lambda^{+}-\Lambda^{-}\right) \partial_{r}+O p\left(\partial_{r} \lambda^{+}\right)-\Lambda^{-} \Lambda^{+} .
$$

So that, by identification of terms of same order

$$
\left\{\begin{array}{l}
\left(\Lambda^{+}-\Lambda^{-}\right) \partial_{r}=\frac{1}{c} O_{B} \\
c O p\left(\partial_{r} \lambda^{+}\right)-\Lambda^{-} \Lambda^{+}=-\partial_{t}^{2}-\mu V \partial_{t}+\frac{c^{2}}{\chi_{r}^{2}} \partial_{\theta}^{2}+O_{F}+O_{D}
\end{array}\right.
$$

so that in term of symbolic computation

$$
\begin{cases}\lambda^{+}-\lambda^{-} & =\frac{q_{O_{B}}}{c} \\ c \partial_{r} \lambda^{+}-\lambda^{-} \# \lambda^{+} & =\tau^{2}-i \mu V \tau-\frac{c^{2}}{\chi_{r}^{2}} \xi^{2}+i q_{O_{F}} \xi+q_{O_{D}} .\end{cases}
$$

It is now possible to identify the sequence $\left(\lambda_{1-j}^{ \pm}\right)_{j}$.

$$
\left\{\begin{array}{l}
\lambda_{1}^{+}-\lambda_{1}^{-}=0 \\
-\lambda_{1}^{-} \lambda_{1}^{+}=\tau^{2}-\frac{c^{2}}{\chi_{r}^{2}} \xi^{2}
\end{array}\right.
$$


This leads to

$$
\left\{\begin{array}{l}
\lambda_{1}^{+}=\lambda_{1}^{-} \\
\lambda_{1}^{+}= \pm\left(-\tau^{2}+\frac{c^{2}}{\chi_{r}^{2}} \xi^{2}\right)^{1 / 2}
\end{array}\right.
$$

We deduce that $\lambda_{1}^{+}=\left(-\tau^{2}+\frac{c^{2}}{\chi_{r}^{2}} \xi^{2}\right)^{1 / 2}$, corresponding to the outgoing bicharacteristic strips, for the same reason as in the laser-free TDKGE. Both equations have indeed the same principal symbol. In addition. Next, and using the explicit formula of $\sigma_{\alpha}\left(\Lambda^{-} \Lambda^{+}\right)$for $\alpha=$ $0,1,2,3$, we successively get

$$
\begin{cases}\lambda_{0}^{+}-\lambda_{0}^{-} & =\frac{q_{O_{B}}}{c} \\ c \partial_{r} \lambda_{1}^{+}-\lambda_{1}^{-} \lambda_{0}^{+}-\lambda_{0}^{-} \lambda_{1}^{+} & =-i \mu V \tau+i q_{O_{F}} \xi .\end{cases}
$$

Then

$$
\begin{aligned}
& \lambda_{0}^{+}=-\frac{1}{2 \lambda_{1}^{+}}\left[-i \mu V \tau+i q_{O_{F}} \xi-c \partial_{r} \lambda_{1}^{+}\right]+\frac{q_{O_{B}}}{2 c} \\
& \lambda_{0}^{-}=-\frac{1}{2 \lambda_{1}^{+}}\left[-i \mu V \tau+i q_{O_{F}} \xi-c \partial_{r} \lambda_{1}^{+}\right]-\frac{q_{O_{B}}}{2 c} .
\end{aligned}
$$

Next,

$$
\begin{cases}\lambda_{-1}^{+}-\lambda_{-1}^{-} & =0 \\ c \partial_{r} \lambda_{0}^{+}-\lambda_{1}^{-} \lambda_{-1}^{+}-\lambda_{0}^{-} \lambda_{0}^{+}-\lambda_{-1}^{-} \lambda_{1}^{+}+\mathrm{i} \partial_{\xi} \lambda_{1}^{-} \partial_{\theta} \lambda_{0}^{+}+\mathrm{i} \partial_{\tau} \lambda_{1}^{-} \partial_{t} \lambda_{0}^{+} & =q_{O_{D}} .\end{cases}
$$

and

$$
\begin{aligned}
& \begin{cases}\lambda_{-2}^{+}-\lambda_{-2}^{-} & =0 \\
c \partial_{r} \lambda_{-1}^{+}-\lambda_{1}^{-} \lambda_{-2}^{+}-\lambda_{0}^{-} \lambda_{-1}^{+}-\lambda_{-1}^{-} \lambda_{0}^{+}-\lambda_{-2}^{-} \lambda_{1}^{+}+i \partial_{\xi} \lambda_{1}^{-} \partial_{\theta} \lambda_{-1}^{+}+ & \\
i \partial_{\tau} \lambda_{1}^{-} \partial_{t} \lambda_{-1}^{+}+i \partial_{\xi} \lambda_{0}^{-} \partial_{\theta} \lambda_{0}^{+}+i \partial_{\tau} \lambda_{0}^{-} \partial_{t} \lambda_{0}^{+}+\frac{1}{2} \partial_{\rho, \xi, \tau}^{2} \lambda_{1}^{-} \partial_{r, \theta, t}^{2} \lambda_{0}^{+}=0 .\end{cases} \\
& \lambda_{-1}^{+}=\frac{1}{2 \lambda_{1}^{+}}\left[-q_{O_{D}}+c \partial_{r} \lambda_{0}^{+}+\mathrm{i} \partial_{\xi} \lambda_{1}^{-} \partial_{\theta} \lambda_{0}^{+}+\mathrm{i} \partial_{\tau} \lambda_{1}^{-} \partial_{t} \lambda_{0}^{+}-\lambda_{0}^{+} \lambda_{0}^{-}\right] .
\end{aligned}
$$

Similarly we deduce

$$
\begin{aligned}
\lambda_{-2}^{+}= & \frac{1}{2 \lambda_{1}^{+}}\left[-\lambda_{-1}^{+}\left[\lambda_{0}^{+}+\lambda_{0}^{-}\right]+c \partial_{r} \lambda_{-1}^{+}+i \partial_{\xi} \lambda_{1}^{-} \partial_{\theta} \lambda_{-1}^{+}+\right. \\
& \left.+i \partial_{\tau} \lambda_{1}^{-} \partial_{t} \lambda_{-1}^{+}+i \partial_{\xi} \lambda_{0}^{-} \partial_{\theta} \lambda_{0}^{+}+i \partial_{\tau} \lambda_{0}^{-} \partial_{t} \lambda_{0}^{+}+\frac{1}{2} \partial_{\rho, \xi, \tau}^{2} \lambda_{1}^{-} \partial_{r, \theta, t}^{2} \lambda_{0}^{+}\right] .
\end{aligned}
$$

As before we can state 
Proposition 2.3. There exists 2 operators $\Lambda_{(1-k, 1)}^{+}, \Lambda_{(1-k, 1)}^{-}$such that

$$
P_{K G L}^{r, \theta}=\left(c \partial_{x}+\Lambda_{(1-k, 1)}^{+}\right)\left(c \partial_{x}-\Lambda_{(1-k, 1)}^{-}\right)+R_{-k}
$$

where $R_{-k} \in O P S^{-k}$ and

$$
\Lambda_{(1-k, 1)}^{+}=O p\left(\sum_{j=0}^{k} \lambda_{1-j}^{+}\right)
$$

where $\left(\lambda_{1-j}^{+}\right)_{j}$, are given by $(24)$.

At this point, we plan to find explicit ABC. We recall $\sqrt{1+x}=1+\frac{1}{2} x-\frac{1}{8} x^{2}+O\left(|x|^{3}\right)$ (for $|x|<1$ ). As a consequence for $\tau$ large (high time-frequency) $\lambda_{1}^{+}$is expanded in:

$$
\begin{aligned}
\lambda_{1}^{+} & =\sqrt{-\tau^{2}\left(1-\frac{c^{2} \xi^{2}}{\chi_{r}^{2} \tau^{2}}\right)} \\
& =i \tau \sqrt{1-\frac{c^{2} \xi^{2}}{\chi_{r}^{2} \tau^{2}}} \\
& =i \tau-\frac{i c^{2} \xi^{2}}{2 \chi_{r}^{2} \tau}-\frac{i c^{4} \xi^{4}}{8 \chi_{r}^{4} \tau^{3}}+O\left(\tau^{-5}\right) .
\end{aligned}
$$

Remark 2.1. In the following, $\lambda_{j}^{ \pm}$will be computed in the regime $\tau \gg 1$.

Next we approximate $\lambda_{0}^{+}$, which is a function of $\partial_{r} \lambda_{1}^{+}$and $\frac{1}{2 \lambda_{1}^{+}}$. First, we easily show that

$$
\begin{aligned}
& \frac{1}{2 \lambda_{1}^{+}}=\frac{1}{2 \mathbf{i} \tau-\frac{c^{2}}{\chi_{r}^{2}} \mathbf{i} \xi^{2} \tau^{-1}} \\
& =-\frac{i}{2} \tau^{-1}-\frac{i c^{2}}{4 \chi_{r}^{2}} \xi^{2} \tau^{-3}+O\left(\tau^{-5}\right)
\end{aligned}
$$

and now the differential term:

$$
c \partial_{r} \lambda_{1}^{+}=\frac{i c^{2} \xi^{2}}{\chi_{r}^{3}} \tau^{-1}=\frac{i c^{4} \xi^{4}}{2 \chi_{r}^{5}} \tau^{-3}+O\left(\tau^{-5}\right) .
$$

As a consequence $\lambda_{0}^{+}$can be approximated by:

$$
\begin{aligned}
\lambda_{0}^{+} & =\frac{1}{2 \lambda_{1}^{+}}\left(-\mathbf{i} \mu V \tau+\mathbf{i} q_{O_{F}} \xi-c \partial_{r} \lambda_{1}^{+}\right)+\frac{q_{O_{B}}}{2 c} \\
& =\left(\frac{i}{2} \tau^{-1}+\frac{i c^{2}}{4 \chi_{r}^{2}} \xi^{2} \tau^{-3}+\frac{i c^{4}}{8 \chi_{r}^{4}} \xi^{4} \tau^{-5}\right)\left(-\mathbf{i} \mu V \tau+\mathbf{i} q_{O_{F}} \xi-\frac{i c^{2} \xi^{2}}{\chi_{r}^{3}} \tau^{-1}-\frac{i c^{4} \xi^{4}}{2 \chi_{r}^{5}} \tau^{-3}\right)+\frac{q_{O_{B}}}{2 c}
\end{aligned}
$$


and

$$
\lambda_{0}^{+}=\frac{\mu}{2} V+\frac{q_{O_{B}}}{2 c}-\frac{q_{O_{F}}}{2} \xi \tau^{-1}+\left(\frac{c^{2}}{2 \chi_{r}^{3}}+\frac{\mu c^{2}}{4 \chi_{r}^{2}} V\right) \xi^{2} \tau^{-2}+O\left(\tau^{-3}\right) .
$$

From there, we derive the expression up to a $O\left(\tau^{-3}\right)$. This choice is motivated by the complexity of the symbolic expression beyond that order, that would lead to complex operators, hard to approximate and that could deteriorate stability and efficiency of the overall scheme. Note that $\lambda_{0}^{-}=\lambda_{0}^{+}-\frac{q_{O_{B}}}{c}$. Next we approximate $\lambda_{-1}^{+}$:

$$
\lambda_{-1}^{+}=\frac{1}{2 \lambda_{1}^{+}}\left(-q_{O_{D}}+c \partial_{r} \lambda_{0}^{+}+i \partial_{\xi} \lambda_{1}^{-} \partial_{\theta} \lambda_{0}^{+}+i \partial_{\tau} \lambda_{1}^{-} \partial_{t} \lambda_{0}^{+}-\lambda_{0}^{+} \lambda_{0}^{-}\right) .
$$

In that goal, we expand $c \partial_{r} \lambda_{0}^{+}$and $\lambda_{0}^{+} \lambda_{0}^{-}$.

$$
c \partial_{r} \lambda_{0}^{+}=\frac{\mu c}{2} \partial_{r} V+\frac{1}{2} \partial_{r} q_{O_{B}}-\frac{c}{2} \partial_{r} q_{O_{F}} \xi \tau^{-1}+\frac{c}{2 \chi_{r}^{2}}\left(-\frac{3 c^{2}}{\chi_{r}^{2}}-\frac{\mu c^{2}}{\chi_{r}} V+\frac{\mu c^{2}}{2} \partial_{r} V\right) \xi^{2} \tau^{-2}+O\left(\tau^{-3}\right)
$$

and by truncating at $O\left(\tau^{-3}\right)$

$$
\lambda_{0}^{+} \lambda_{0}^{-}=\frac{\mu^{2}}{4} V^{2}-\frac{q_{O_{B}}^{2}}{4 c^{2}}-\frac{\mu}{2} q_{O_{F}} V \xi \tau^{-1}+\left(\mu V q_{O_{H}}+\frac{q_{O_{F}}^{2}}{4}\right) \xi^{2} \tau^{-2}+O\left(\tau^{-3}\right) .
$$

Where $q_{O_{H}}:=\frac{c^{2}}{2 \chi_{r}^{3}}+\frac{\mu c^{2}}{4 \chi_{r}^{2}} V$. In addition,

$$
i \partial_{\xi} \lambda_{1}^{-} \partial_{\theta} \lambda_{0}^{+}=\left(\frac{\mu}{2} \partial_{\theta} V+\frac{\partial_{\theta} q_{O_{B}}}{2 c}\right) \frac{c^{2}}{\chi_{r}^{2}} \xi \tau^{-1}-\frac{c^{2} \partial_{\theta} q_{O_{F}}}{2 \chi_{r}^{2}} \xi^{2} \tau^{-2}+O\left(\tau^{-3}\right)
$$

and

$$
\begin{aligned}
i \partial_{\tau} \lambda_{1}^{-} \partial_{t} \lambda_{0}^{+}= & -\frac{\mu}{2} \partial_{t} V-\frac{\partial_{t} q_{O_{B}}}{2 c}+\frac{\partial_{t} q_{O_{F}}}{2} \xi \tau^{-1}-\frac{c^{2}}{2 \chi_{r}^{2}}\left(\frac{\mu}{2} \partial_{t} V+\frac{\partial_{t} q_{O_{B}}}{2 c}\right) \xi^{2} \tau^{-2}+O\left(\tau^{-3}\right) . \\
\lambda_{-1}^{+}= & \left(-\frac{i}{2} \tau^{-1}+O\left(\tau^{-3}\right)\right)\left[-q_{O_{D}}-\frac{\mu}{2} \partial_{t} V-\frac{\partial_{t} q_{O_{B}}}{2 c}+\frac{\mu c}{2} \partial_{r} V+\frac{1}{2} \partial_{r} q_{O_{B}}\right. \\
& -\frac{\mu^{2}}{4} V^{2}+\frac{q_{O_{B}}^{2}}{4 c^{2}}-\frac{c}{2} \partial_{r} q_{O_{F}} \xi \tau^{-1}+\left(\frac{\mu}{2} \partial_{\theta} V+\frac{\partial_{\theta} q_{O_{B}}}{2 c}\right) \frac{c^{2}}{\chi_{r}^{2}} \xi \tau^{-1}+\frac{\partial_{t} q_{O_{F}}}{2} \xi \tau^{-1} \\
+ & \left.\frac{\mu}{2} q_{O_{F}} V \xi \tau^{-1}+O\left(\tau^{-2}\right)\right] .
\end{aligned}
$$

We now set

$$
\left\{\begin{aligned}
q_{O_{I}} & :=\frac{i q_{O_{D}}}{2}-\frac{i \mu c}{4} \partial_{r} V-\frac{i}{4} \partial_{r} q_{O_{B}}+\frac{i \mu^{2}}{8} V^{2}-\frac{i q_{O_{B}}^{2}}{8 c^{2}}+\frac{i \mu}{4} \partial_{t} V+\frac{i}{4 c} \partial_{t} q_{O_{B}} \\
q_{O_{J}} & :=\frac{i c}{4} \partial_{r} q_{O_{F}}-\frac{i \mu q_{O_{F}}}{4} V-\frac{i}{4} \partial_{t} q_{O_{F}}-\left(\frac{\mu}{2} \partial_{\theta} V+\frac{\partial_{\theta} q_{O_{B}}}{2 c}\right) \frac{i c^{2}}{2 \chi_{r}^{2}} .
\end{aligned}\right.
$$


$\lambda_{-1}^{+}$can finally be written truncating the terms higher than $\tau^{-3}$ :

$$
\lambda_{-1}^{+}=q_{O_{I}} \tau^{-1}+q_{O_{J}} \xi \tau^{-2}+O\left(\tau^{-3}\right) .
$$

Again truncating terms higher than $O\left(\tau^{-3}\right)$ from

$$
\begin{aligned}
\lambda_{-2}^{+}= & \frac{1}{2 \lambda_{1}^{+}}\left[-\lambda_{-1}^{+}\left[\lambda_{0}^{+}+\lambda_{0}^{-}\right]+c \partial_{r} \lambda_{-1}^{+}+\mathrm{i} \partial_{\xi} \lambda_{1}^{-} \partial_{\theta} \lambda_{-1}^{+}+\right. \\
& \left.+\mathrm{i} \partial_{\tau} \lambda_{1}^{-} \partial_{t} \lambda_{-1}^{+}+\mathrm{i} \partial_{\xi} \lambda_{0}^{-} \partial_{\theta} \lambda_{0}^{+}+\mathrm{i} \partial_{\tau} \lambda_{0}^{-} \partial_{t} \lambda_{0}^{+}+\frac{1}{2} \partial_{\rho, \xi, \tau}^{2} \lambda_{1}^{-} \partial_{r, \theta, t}^{2} \lambda_{0}^{+}\right] .
\end{aligned}
$$

We note that $\lambda_{0}^{+}+\lambda_{0}^{-}=2 \lambda_{0}^{+}-\frac{q_{O_{B}}}{c}$. We set

$$
\begin{aligned}
q_{O_{G}} & =2 \lambda_{0}^{+}-\frac{q_{O_{B}}}{c} \\
& =\mu V-q_{O_{F}} \xi \tau^{-1}+\left(\frac{c^{2}}{\chi_{r}^{3}}+\frac{\mu c^{2}}{2 \chi_{r}^{2}} V\right) \xi^{2} \tau^{-2}+O\left(\tau^{-3}\right) .
\end{aligned}
$$

Moreover

$$
\left\{\begin{aligned}
\mathrm{i} \partial_{\xi} \lambda_{1}^{-} \partial_{\theta} \lambda_{-1}^{+}= & \frac{c^{2}}{\chi_{r}^{2}} \partial_{\theta} q_{O_{I}} \xi \tau^{-2}+O\left(\tau^{-3}\right) \\
\mathrm{i} \partial_{\tau} \lambda_{1}^{-} \partial_{t} \lambda_{-1}^{+}= & -\partial_{t} q_{O_{I}} \tau^{-1}-\partial_{t} q_{O_{J}} \xi \tau^{-2}+O\left(\tau^{-3}\right) \\
\mathrm{i} \partial_{\xi} \lambda_{0}^{-} \partial_{\theta} \lambda_{0}^{+}= & -\frac{i q_{O_{F}}}{2}\left(\frac{\mu}{2} \partial_{\theta} V+\frac{\partial_{\theta} q_{O_{B}}}{2 c}\right) \tau^{-1} \\
& +\left(\frac{\mathrm{i} q_{O_{F}} \partial_{\theta} q_{O_{F}}}{4}+2 \mathrm{i}\left(\frac{\mu}{2} \partial_{\theta} V+\frac{\partial_{\theta} q_{O_{B}}}{2 c}\right)\left(\frac{c^{2}}{2 \chi_{r}^{3}}+\frac{\mu c^{2}}{4 \chi_{r}^{2}} V\right)\right) \xi \tau^{-2}+O\left(\tau^{-3}\right) \\
\mathrm{i} \partial_{\tau} \lambda_{0}^{-} \partial_{t} \lambda_{0}^{+}= & \frac{i q_{O_{F}}}{2}\left(\frac{\mu}{2} \partial_{t} V+\frac{\partial_{t} q_{O_{B}}}{2 c}\right) \xi \tau^{-2}+O\left(\tau^{-3}\right) .
\end{aligned}\right.
$$

Finally we observe that $\partial_{\rho, \xi, \tau}^{2} \lambda_{1}^{-} \partial_{r, \theta, t}^{2} \lambda_{0}^{+} / 2=O\left(\tau^{-3}\right)$. We now deduce that

$$
\lambda_{-2}^{+}=\left(\frac{\dot{i}}{2}\left(q_{O_{I}} q_{O_{G}}-c \partial_{r} q_{O_{I}}+\partial_{t} q_{O_{I}}\right)-\frac{q_{O_{F}}}{4}\left(\frac{\mu}{2} \partial_{\theta} V+\frac{\partial_{\theta} q_{O_{B}}}{2 c}\right)\right) \tau^{-2} .
$$


It is now possible to write out the whole approximation

$$
\begin{aligned}
\sum_{j=0}^{3} \lambda_{1-j}^{+}= & \mathbf{i} \tau-\frac{i c^{2}}{2 \chi_{r}^{2}} \xi^{2} \tau^{-1}+\frac{\mu}{2} V+\frac{q_{O_{B}}}{2 c}-\frac{q_{O_{F}}}{2} \xi \tau^{-1} \\
& +\left(\frac{c^{2}}{2 \chi_{r}^{3}}+\frac{\mu c^{2}}{4 \chi_{r}^{2}} V\right) \xi^{2} \tau^{-2}+q_{O_{I}} \tau^{-1}+q_{O_{J}} \xi \tau^{-2} \\
& +\left(\frac{i}{2}\left(q_{O_{I}} q_{O_{G}}-c \partial_{r} q_{O_{I}}+\partial_{t} q_{O_{I}}\right)-\frac{q_{O_{F}}}{4}\left(\frac{\mu}{2} \partial_{\theta} V+\frac{\partial_{\theta} q_{O_{B}}}{2 c}\right)\right) \tau^{-2}+O\left(\tau^{-3}\right) \\
= & \mathbf{i} \tau+\frac{\mu}{2} V+\frac{q_{O_{B}}}{2 c}+\left(-\frac{i c^{2}}{2 \chi_{r}^{2}} \xi^{2}-\frac{q_{O_{F}}}{2} \xi+q_{O_{I}}\right) \tau^{-1} \\
& +\left(\left(\frac{c^{2}}{2 \chi_{r}^{3}}+\frac{\mu c^{2}}{4 \chi_{r}^{2}} V\right) \xi^{2}+q_{O_{J}} \xi+\frac{i}{2}\left(q_{O_{I}} q_{O_{G}}-c \partial_{r} q_{O_{I}}+\partial_{t} q_{O_{I}}\right)\right. \\
& \left.-\frac{q_{O_{F}}}{4}\left(\frac{\mu}{2} \partial_{\theta} V+\frac{\partial_{\theta} q_{O_{B}}}{2 c}\right)\right) \tau^{-2}+O\left(\tau^{-3}\right) .
\end{aligned}
$$

Denoting by $\lambda_{(-2,1)}^{+}:=\sum_{j=0}^{3} \lambda_{1-j}^{+}$, which is an approximation of $\lambda^{+}$. Going back to operators, $\Lambda_{(-2,1)}^{+}$is given by ${ }^{1}$

$$
\begin{aligned}
\Lambda_{(-2,1)}^{+}= & \partial_{t}+\frac{\mu}{2} V+\frac{1}{2 c} O_{B}+\left(-\frac{c^{2}}{2 \chi_{r}^{2}} \partial_{\theta}^{2}-\frac{1}{2} O_{F} \partial_{\theta}+i O_{I}\right) \partial_{t}^{-1}-\left(\left(-\frac{c^{2}}{2 \chi_{r}^{3}}+\frac{\mu c^{2}}{4 \chi_{r}^{2}} V\right) \partial_{\theta}^{2}\right. \\
& \left.-i O_{J} \partial_{\theta}+\frac{i}{2}\left(O_{I} O_{G}-c \partial_{r} O_{I}+\partial_{t} O_{I}\right)-\frac{1}{4} O_{F}\left(\frac{\mu}{2} \partial_{\theta} V+\frac{\partial_{\theta} O_{B}}{2 c}\right)\right) \partial_{t}^{-2} .
\end{aligned}
$$

We have proven the following proposition

Proposition 2.4. For $\tau$ sufficiently large, a family of $A B C$ for laser-particle TDKGE is given by

$$
\left(c \partial_{r}+\Lambda_{(1-j)}^{+}\right) \varphi=0, \quad j=1,2,3,4
$$

where for

- $j=0$ :

$$
\Lambda_{(1,1)}^{+}=\partial_{t}
$$

- $j=1$ :

$$
\Lambda_{(0,1)}^{+}=\Lambda_{(1,1)}^{+}+\frac{\mu}{2} V+\frac{1}{2 c} O_{B}
$$

\footnotetext{
${ }^{1} O$. stand for $o p\left(q_{O}.\right)$
} 
- $j=2$ :

$$
\Lambda_{(-1,1)}^{+}=\Lambda_{(0,1)}^{+}+\left(-\frac{c^{2}}{2 \chi_{r}^{2}} \partial_{\theta}^{2}-\frac{1}{2} O_{F} \partial_{\theta}+i O_{I}\right) \partial_{t}^{-1}
$$

- $j=3$ :

$$
\begin{aligned}
\Lambda_{(-2,1)}^{+}= & \Lambda_{(-1,1)}^{+}-\left(-\left(\frac{c^{2}}{2 \chi_{r}^{3}}+\frac{\mu c^{2}}{4 \chi_{r}^{2}} V\right) \partial_{\theta}^{2}-\mathbf{i} q_{O_{J}} \partial_{\theta}\right. \\
& \left.+\frac{\dot{i}}{2}\left(O_{I} O_{G}-c \partial_{r} O_{I}+\partial_{t} O_{I}\right)-\frac{1}{4} O_{F}\left(\frac{\mu}{2} \partial_{\theta} V+\frac{\partial_{\theta} O_{B}}{2 c}\right)\right) \partial_{t}^{-2} .
\end{aligned}
$$

In practice, the order of the $\mathrm{ABC}$ will be chosen in accordance with the order of accuracy of the scheme which is used to solve the TDKGE inside the domain $\Omega$.

\section{Absorbing Boundary Conditions for Dirac Equation in 2-d}

In this section, we are interested in the derivation of ABC for the 2-d TDDE. The strategy which is used is slightly different from the one used for TDKGE, although some close connections exist and will be discussed later. The idea here is to apply a diagonalization procedure as usually done for hyperbolic systems. See for instance [15].

\subsection{Dirac equation}

We denote by $P_{D}$ the 2-dimensional Dirac operator such that $P_{D} \mathbf{u}=\mathbf{0}$ in a bounded domain $\Omega$, and as before we derive a condition on $\partial \Omega$ to avoid spurious wave reflections.

$$
P_{D}:=\partial_{t}+\alpha_{x}\left(c \partial_{x}-\mathrm{i} e A_{x}\right)+\alpha_{y}\left(c \partial_{y}-\mathrm{i} e A_{y}\right)+\mathrm{i}\left(V_{c}+e V\right) \mathbf{I}_{4}-\mathrm{i} \beta m c^{2}
$$

where

- $V_{c}: \Omega \rightarrow \mathbb{R}$ is a Coulomb potential.

- $V: \Omega \times \mathbb{R}_{+} \rightarrow \mathbb{R}$ is a combination of the self-consistent and external electric potentials.

- $\mathbf{A}: \Omega \times \mathbb{R}_{+} \rightarrow \mathbb{R}^{2}$ is a combination of the electromagnetic potential generated by the particle charge and by an external potential.

- $m$ is the mass of the particle, $e$ their charge, $c$ the speed of light.

- $\mathbf{u}: \mathbb{R}_{+} \times \Omega \rightarrow \mathbb{C}^{4}$ is the Dirac wavefunction, that is a spinor field initially normalized to 1: $\|\mathbf{u}(0, \cdot)\|_{\left(L^{2}(\Omega)\right)^{4}}=1$. 
- Hermitian Dirac matrices $\alpha_{x}, \alpha_{y}, \beta$ are defined by

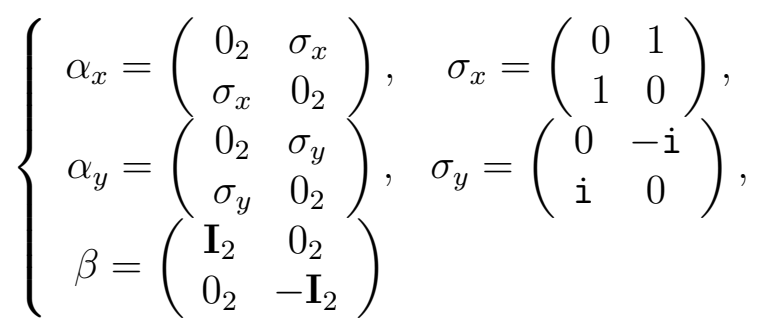

and $0_{2}$ and $\mathbf{I}_{2}$ are respectively the zero and identity matrices in $M_{2}(\mathbb{C})$. The following relations hold: $\alpha_{x}^{2}=\alpha_{y}^{2}=\beta^{2}=I_{4}$ and $\left\{\alpha_{x}, \alpha_{y}\right\}=\left\{\alpha_{x}, \beta\right\}=\left\{\alpha_{y}, \beta\right\}=0_{4}$.

- $\mathbf{J}=\left(J_{x}, J_{y}\right)$ with $J_{x}=e c\left(\mathbf{u}, \alpha_{x} \mathbf{u}\right)_{\mathbb{C}^{4}}, J_{y}=e c\left(\mathbf{u}, \alpha_{y} \mathbf{u}\right)_{\mathbb{C}^{4}}$, denotes the current density and $\rho$ denotes the particle density which is equal to $e \sum_{i=1}^{4}\left|\mathbf{u}_{i}\right|^{2}$.

In the following, we will assume that $(\mathbf{A}, V)$ is given at any time.

\subsection{Laser-particle TDDE in 2-d}

As before, we consider a circular domain $\Omega_{R}$ of radius $R$, and the system is rewritten at the boundary in polar coordinates, where $r \in[0, \varepsilon)$ with $\varepsilon>0$ :

$$
\left(\partial_{r}+L\left(r, \theta, t, \partial_{\theta}, \partial_{t}\right)\right) \mathbf{u}=\mathbf{0}
$$

with $L=L_{1}+L_{0} \in O P S^{1}$ and $L_{i}$ is an operator of order $i(=0,1)$. We denote $\mathbb{R}_{+}^{2}:=$ $\mathbb{R}_{+}^{*} \times[0, T]$ and $\nu_{\left(x_{0}, y_{0}\right)}$ a neighborhood of $\left(x_{0}, y_{0}\right)$. Finally we denote $\Sigma_{T}^{R}:=\partial \Omega_{R} \times[0, T]$ and $\Sigma_{T}^{R+r}=\partial \Omega_{R+r}^{+} \times[0, T]$ with $\Omega_{R+r}^{+}$a disc of radius $R+r$ containing $\Omega_{R}$. We then study the TDDE in the region $\Omega_{R+r}^{+}-\Omega_{R}$ and design boundary conditions such that incoming waves at $r=0$ are set to zero or equivalently to vanish waves that leave the crown surrounding $\Omega_{R}$. Some basic computations lead on $\Sigma_{T}^{R+r}$ to

$$
L_{1}:=\frac{1}{c} \tilde{\alpha}_{x} \partial_{t}+\frac{1}{\chi_{r}} \tilde{\alpha}_{x} \tilde{\alpha}_{y} \partial_{\theta}, L_{0}:=\frac{1}{c} \tilde{\alpha}_{x} \tilde{\beta}
$$

with $\chi_{r}=\frac{1}{R+r}$ and

$$
\tilde{\alpha}_{x}=\left(\begin{array}{cccc}
0 & 0 & 0 & e^{-\mathbf{i} \theta} \\
0 & 0 & e^{\mathrm{i} \theta} & 0 \\
0 & e^{-\mathrm{i} \theta} & 0 & 0 \\
e^{\mathrm{i} \theta} & 0 & 0 & 0
\end{array}\right)
$$

and

$$
\tilde{\alpha}_{y}=\left(\begin{array}{cccc}
0 & 0 & 0 & -i e^{-i \theta} \\
0 & 0 & i e^{i \theta} & 0 \\
0 & -i e^{-i \theta} & 0 & 0 \\
i e^{i \theta} & 0 & 0 & 0
\end{array}\right)
$$


and

$$
\tilde{\beta}=i \beta m c^{2}+i\left(e V+V_{c}\right) \mathbf{I}_{4}-i e\left(A_{r} \tilde{\alpha}_{x}+A_{\theta} \tilde{\alpha}_{y}\right)
$$

that is

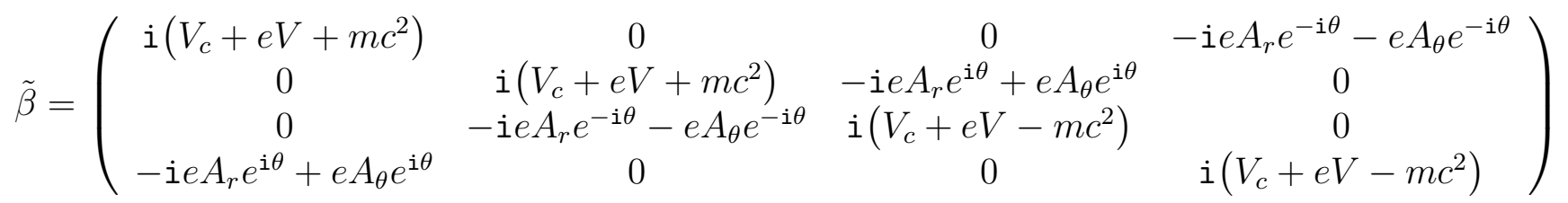

Now as

$$
\tilde{\alpha}_{x} \tilde{\alpha}_{y}=\left(\begin{array}{cccc}
i & 0 & 0 & 0 \\
0 & -i & 0 & 0 \\
0 & 0 & i & 0 \\
0 & 0 & 0 & -i
\end{array}\right)
$$

and $\tilde{\alpha}_{x} \tilde{\beta}=$

$$
\left(\begin{array}{cccc}
-\mathrm{i} e A_{r}+e A_{\theta} & 0 & 0 & \mathrm{i} e^{-\mathrm{i} \theta}\left(V_{c}+e V-m c^{2}\right) \\
0 & -\mathrm{i} e A_{r}-e A_{\theta} & \mathrm{i} e^{\mathrm{i} \theta}\left(V_{c}+e V-m c^{2}\right) & 0 \\
0 & \mathrm{i} e^{-\mathrm{i} \theta}\left(V_{c}+e V+m c^{2}\right) & -\mathrm{i} e A_{r}+e A_{\theta} & 0 \\
\mathrm{i} e^{\mathrm{i} \theta}\left(V_{c}+e V+m c^{2}\right) & 0 & 0 & -\mathrm{i} e A_{r}-e A_{\theta}
\end{array}\right)
$$

we get, denoting by $\mathcal{L}_{1}=\sigma\left(L_{1}\right)$,

$$
\mathcal{L}_{1}=\left(\begin{array}{cccc}
-\frac{\omega}{\chi_{r}} & 0 & 0 & \frac{\mathrm{i} \tau}{c} e^{-\mathrm{i} \theta} \\
0 & \frac{\omega}{\chi_{r}} & \frac{\mathbf{i} \tau}{c} e^{\mathrm{i} \theta} & 0 \\
0 & \frac{\mathrm{i} \tau}{c} e^{-\mathrm{i} \theta} & -\frac{\omega}{\chi_{r}} & 0 \\
\frac{\mathrm{i} \tau}{c} e^{\mathrm{i} \theta} & 0 & 0 & \frac{\omega}{\chi_{r}}
\end{array}\right)
$$

and $\tilde{\mathcal{L}}:=\mathbf{i} \rho \mathbf{I}_{4}+\mathcal{L}_{1}$. The leading symbol $p_{1}$ is defined by

$$
p_{1}(r, \theta, t, \rho, \xi, \omega)=\operatorname{det}(\tilde{\mathcal{L}}(r, \theta, t, \xi, \omega))
$$

where

$$
\tilde{\mathcal{L}}=\left(\begin{array}{cccc}
\mathbf{i} \rho-\frac{\omega}{\chi_{r}} & 0 & 0 & \frac{\mathbf{i} \tau}{c} e^{-\mathbf{i} \theta} \\
0 & \mathbf{i} \rho+\frac{\omega}{\chi_{r}} & \frac{\mathrm{i} \tau}{c} e^{\mathrm{i} \theta} & 0 \\
0 & \frac{\mathrm{i} \tau}{c} e^{-\mathbf{i} \theta} & \mathrm{i} \rho-\frac{\omega}{\chi_{r}} & 0 \\
\frac{\mathrm{i} \tau}{c} e^{\mathrm{i} \theta} & 0 & 0 & \mathrm{i} \rho+\frac{\omega}{\chi_{r}}
\end{array}\right)
$$


$p_{1}$ has 2 double real roots

$$
\rho^{ \pm}= \pm\left(\frac{\tau^{2}}{c^{2}}-\frac{\omega^{2}}{\chi_{r}^{2}}\right)^{1 / 2}
$$

in the hyperbolic region of the cotangent bundle, defined by

$$
\mathcal{H}\left(\Sigma_{T}^{R+r}\right)=\left\{(\theta, t, \omega, \tau) \in T^{*}\left(\Sigma_{T}^{R+r}\right):\left(\frac{\omega^{2}}{\chi_{r}^{2}}-\frac{\tau^{2}}{c^{2}}\right)<0\right\} \subseteq T^{*}\left(\Sigma_{T}^{R+r}\right)
$$

as

$$
p_{1}(r, \theta, t, \rho, \xi, \omega)=\left(\rho^{2}-\frac{\tau^{2}}{c^{2}}+\frac{\omega^{2}}{\chi_{r}^{2}}\right)^{2}
$$

In $\mathcal{H}\left(\Sigma_{T}^{R+r}\right)$, the outgoing bicharacteristic correspond to $r^{\prime}(s)>0$, that is

$$
r^{\prime}(s)=\frac{\partial p_{1}}{\partial \rho}=4 \rho\left(\rho^{2}-\left(\frac{\tau^{2}}{c^{2}}-\frac{\omega^{2}}{\chi_{r}^{2}}\right)\right)(s)
$$

So that $r^{\prime}(s)>0$ iff

$$
\left(\rho>0 \text { and } \rho^{2}>\left(\frac{\tau^{2}}{c^{2}}-\frac{\omega^{2}}{\chi_{r}^{2}}\right)\right) \text { or }\left(\rho<0 \text { and } \rho^{2}<\left(\frac{\tau^{2}}{c^{2}}-\frac{\omega^{2}}{\chi_{r}^{2}}\right)\right)
$$

in a small conic neighborhood of $\mathcal{H}\left(\Sigma_{T}^{R}\right)$. We denote by $\mathcal{N}_{0}$ the transition matrix such that $\mathcal{L}_{1}=\mathcal{N}_{0} \sigma\left(\Lambda_{1}\right) \mathcal{N}_{0}^{-1}$ where

$$
\sigma\left(\Lambda_{1}\right)=\left(\begin{array}{cccc}
-i\left(\frac{\tau^{2}}{c^{2}}-\frac{\omega^{2}}{\chi_{r}^{2}}\right)^{1 / 2} & 0 & 0 & 0 \\
0 & -i\left(\frac{\tau^{2}}{c^{2}}-\frac{\omega^{2}}{\chi_{r}^{2}}\right)^{1 / 2} & 0 & 0 \\
0 & 0 & i\left(\frac{\tau^{2}}{c^{2}}-\frac{\omega^{2}}{\chi_{r}^{2}}\right)^{1 / 2} & 0 \\
0 & 0 & 0 & i\left(\frac{\tau^{2}}{c^{2}}-\frac{\omega^{2}}{\chi_{r}^{2}}\right)^{1 / 2}
\end{array}\right)
$$

and

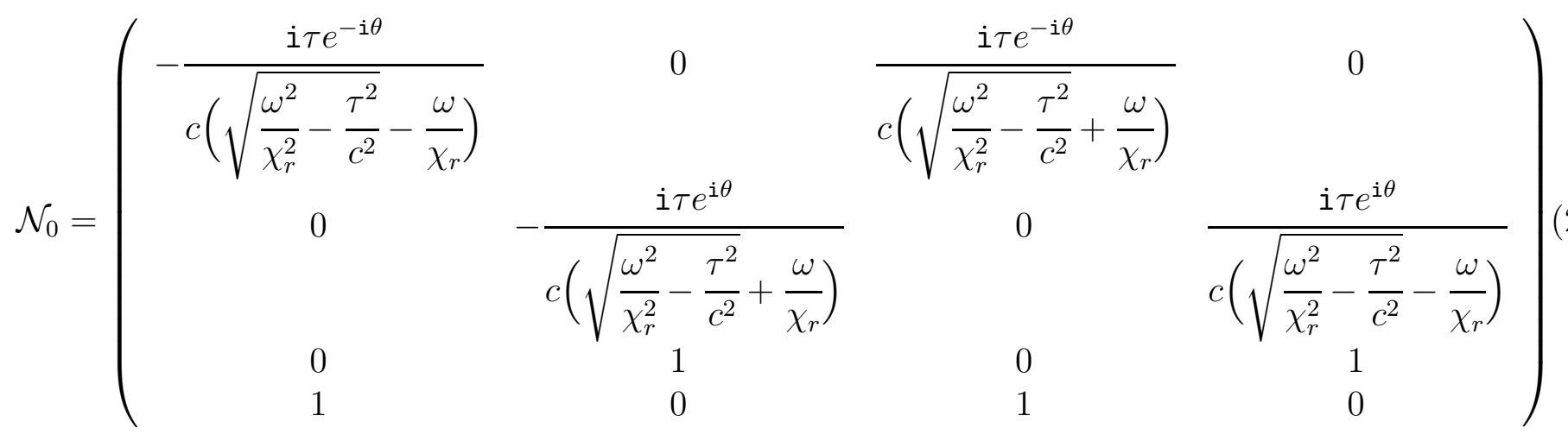


We denote by $V_{0}$ the operator $O p\left(\mathcal{N}_{0}\right) \in O P S^{0}$, with naturally $V_{0} V_{0}^{-1}=I+R$ and $R \in$ $O P S^{-\infty}$. We apply a technique originally proposed by Taylor, and developed in details by Antoine et al. in [2] for electromagnetism. Starting from $\left(\frac{\partial}{\partial r}+L\right) \mathbf{u}=\mathbf{0}$, we set $\mathbf{w}:=V_{0}^{-1} \mathbf{u}$. The equation becomes

$$
\left(\frac{\partial}{\partial r}+\Lambda_{1}\right) \mathbf{w}_{0}=R_{0} \mathbf{w}_{0}
$$

where

$$
R_{0}:=-V_{0}^{-1} L_{0} V_{0}-V_{0}^{-1} \frac{\partial V_{0}}{\partial r}
$$

From there, Taylor's method can be implemented. The principle consists of block-diagonalizing the original system up to a certain order. More precisely, we search successive approximations of the solution, in the form of a sequence

$$
\frac{\partial \mathbf{w}_{-m}}{\partial r}+\sum_{j=-1}^{m-1} \Lambda_{-j} \mathbf{w}_{-m}=R_{-m} \mathbf{w}_{-m}
$$

where $\mathbf{w}_{-m}=\left(I+K_{-m}\right) \mathbf{w}_{-m+1}(m \geqslant 1), \Lambda_{-m+1} \in O P S^{-m+1}$ and $R_{-m} \in O P S^{-m}$. In fine, and according to [28], [2], we have

Proposition 3.1. The solution $\mathbf{u}$ to $(26)$, is such that $\mathbf{w} \sim V \mathbf{u}$, where the solution $\mathbf{w}$ to

$$
\left(\frac{\partial}{\partial r}+\Lambda\right) \mathbf{w} \sim \mathbf{0}
$$

is given by $\Pi_{p=m+1}^{\infty}\left(I+K_{-p}\right) \mathbf{w}_{-m}$, where

$$
\sigma(\Lambda)=\left(\begin{array}{cc}
\sigma\left(\Lambda^{-}\right) & 0 \\
0 & \sigma\left(\Lambda^{+}\right)
\end{array}\right) \in M_{4}(\mathbb{C})
$$

and $\Lambda^{ \pm}$are 2 first order operators of diagonal principal symbol. In addition, Operator $V$ is defined as $\Pi_{j=-\infty}^{1}\left(I+K_{-j}\right) V_{0}^{-1}$, for $K_{-j} \in O P S^{-j}$. Sign $\sim$ is to be understood, as equality up to a smoothing or regularizing operator.

This is equivalent to say that the operator $P_{D}=\partial_{r}+L$ can in fact be approximately blockdiagonalized, or equivalently can be rewritten in the form $\left(\partial_{r}+\Lambda\right)+R$, via the "transition operator" $V$, where $R, V$ and $\Lambda$ are defined explicitly in the proposition above.

We now explicitely implement the diagonalizing method, and we first search for an operator $K_{-1} \in O P S^{-1}$ with $\mathcal{K}_{-1}=\sigma\left(K_{-1}\right)$, such that $\mathbf{w}_{-1}$ is defined by

$$
\mathbf{w}_{-1}=\left(I+K_{-1}\right) \mathbf{w}_{0}
$$


and $\mathbf{w}_{-1}$ satisfies the equation

$$
\frac{\partial \mathbf{w}_{-1}}{\partial r}+\left(\Lambda_{0}+\Lambda_{1}\right) \mathbf{w}_{-1}=R_{-1} \mathbf{w}_{-1}
$$

According to $[2]$

$$
\left(\mathcal{K}_{-1}\right)_{i j}= \begin{cases}\frac{\sigma_{0}\left(R_{0}\right)_{i j}}{\lambda_{1, j}-\lambda_{1, i}} & \forall(i, j) \in \mathcal{J}_{n} \\ 0 & \forall(i, j) \in \mathcal{J}_{d}\end{cases}
$$

where

$$
\left\{\begin{array}{l}
\mathcal{J}_{n}:=\{(1,3),(1,4),(2,3),(2,4),(3,1),(3,2),(4,1),(4,2)\} \\
\mathcal{J}_{d}:=\{(1,1),(1,2),(2,1),(2,2),(3,3),(3,4),(4,3),(4,4)\}
\end{array}\right.
$$

and, denoting $\mathcal{R}_{0}=\sigma_{0}\left(R_{0}\right)$ which represents the order 0 part of $R_{0}$.

$$
\left(\Lambda_{0}\right)_{i j}=\left\{\begin{array}{l}
\left(\mathcal{R}_{0}\right)_{i j}, \quad \forall(i, j) \in \mathcal{J}_{d} \\
0, \quad \forall(i, j) \in \mathcal{J}_{n}
\end{array}\right.
$$

By construction, see [2],

$$
\left(\Lambda_{-m+1}\right)_{(i, j)}= \begin{cases}\left.\left(-\left[K_{-m}, \Lambda_{1}\right]+O p\left(\sigma_{-m+1}\left(R_{-m+1}\right)\right)\right]\right)_{i j}, & \forall(i, j) \in \mathcal{J}_{d} \\ 0, & \forall(i, j) \in \mathcal{J}_{n}\end{cases}
$$

and

$$
\frac{\partial \mathbf{w}_{-m}}{\partial r}+\sum_{j=-1}^{m-1} \Lambda_{-j} \mathbf{w}_{-m}=R_{-m} \mathbf{w}_{-m}
$$

where $\sum_{j=-1}^{m-1} \Lambda_{-j}$ is a block diagonal first order operator with principal symbol

$$
\sigma_{1}\left(\sum_{j=-1}^{m-1} \Lambda_{-j}\right)=\lambda\left(\begin{array}{cccc}
-1 & 0 & 0 & 0 \\
0 & -1 & 0 & 0 \\
0 & 0 & 1 & 0 \\
0 & 0 & 0 & 1
\end{array}\right)
$$

where we have set

$$
\lambda:=i\left(\frac{\tau^{2}}{c^{2}}-\frac{\omega^{2}}{\chi_{r}^{2}}\right)^{1 / 2}
$$

In fine, $\mathrm{ABC}$ are deduced from

$$
\frac{\partial \mathbf{w}_{-m}^{-}}{\partial r}+\Lambda^{-} \mathbf{w}_{-m}=\mathbf{0}
$$


where $\Lambda^{-} \in M_{2}(\mathbb{C})$

$$
\Lambda^{-}=\left\{\begin{array}{l}
\left(\sum_{j=-1}^{m-1} \Lambda_{-j}\right)_{k l}, \text { for }(k, l) \in\{(1,1),(1,2),(2,1),(2,2)\} \\
0, \text { otherwise }
\end{array}\right.
$$

and

$$
\mathbf{w}=\Pi_{p=m+1}^{\infty}\left(I+K_{-p}\right) \mathbf{w}_{-m}
$$

where for $\mathbf{w}=\left(w_{1}, w_{2}, w_{3}, w_{4}\right) \in \mathbb{C}^{4}$.

At this point we have to evaluate $\mathcal{R}_{0}$. We again refer to [2] for a detailed description of algebraic symbolic computations. We just apply these formulas skipping the computational details. That is

$$
\sigma_{0}\left(V_{0}^{-1} \frac{\partial V_{0}}{\partial r}\right)=\mathcal{N}_{0}^{-1}\left(\frac{\partial \mathcal{N}_{0}}{\partial r}\right)
$$

We now have to estimate $\sigma_{0}\left(V_{0}^{-1} L V_{0}\right)$. We first easily prove that

$$
\sigma_{0}\left(V_{0}^{-1} L V_{0}\right)=\mathcal{N}_{0}^{-1} \mathcal{L}_{0} \mathcal{N}_{0}
$$

where

$$
\mathcal{N}_{0}^{-1}=\left(\begin{array}{cccc}
\frac{1}{\alpha-\beta} & 0 & 0 & \frac{\beta}{\beta-\alpha} \\
0 & \frac{e^{-2 \mathrm{i} \theta}}{\alpha-\beta} & \frac{\alpha}{\alpha-\beta} & 0 \\
\frac{1}{\beta-\alpha} & 0 & 0 & \frac{\alpha}{\alpha-\beta} \\
0 & \frac{e^{-2 \mathrm{i} \theta}}{\beta-\alpha} & \frac{\beta}{\beta-\alpha} & 0
\end{array}\right)
$$

and

$$
\left\{\begin{array}{l}
\alpha:=\frac{i \tau e^{-\mathbf{i} \theta}}{c\left(\frac{\omega}{\chi_{r}}-\sqrt{\left.\frac{\omega^{2}}{\chi_{r}^{2}}-\frac{\tau^{2}}{c^{2}}\right)}\right.} \\
\beta:=\frac{\mathbf{i} \tau e^{-\mathbf{i} \theta}}{c\left(\frac{\omega}{\chi_{r}}+\sqrt{\frac{\omega^{2}}{\chi_{r}^{2}}-\frac{\tau^{2}}{c^{2}}}\right)}
\end{array}\right.
$$


that could be rewritten after simplifications

$$
\mathcal{N}_{0}^{-1}=\left(\begin{array}{cccc}
-\frac{\mathrm{i} \tau e^{\mathrm{i} \theta}}{2 c \sqrt{\frac{\omega^{2}}{\chi_{r}^{2}}-\frac{\tau^{2}}{c^{2}}}} & 0 & 0 & -\frac{\tau^{2}}{2 c^{2} \sqrt{\frac{\omega^{2}}{\chi_{r}^{2}}+\frac{\tau^{2}}{c^{2}}}\left(\frac{\omega}{\chi_{r}}-\sqrt{\frac{\omega^{2}}{\chi_{r}^{2}}-\frac{\tau^{2}}{c^{2}}}\right)} \\
0 & -\frac{\mathrm{i} \tau e^{-\mathrm{i} \theta}}{2 c \sqrt{\frac{\omega^{2}}{\chi_{r}^{2}}-\frac{\tau^{2}}{c^{2}}}} & \frac{\tau^{2}}{2 c^{2} \sqrt{\frac{\omega^{2}}{\chi_{r}^{2}}-\frac{\tau^{2}}{c^{2}}}\left(\frac{\omega}{\chi_{r}}-\sqrt{\frac{\omega^{2}}{\chi_{r}^{2}}-\frac{\tau^{2}}{c^{2}}}\right)} & 0 \\
\frac{\mathrm{i} \tau e^{\mathrm{i} \theta}}{2 c \sqrt{\frac{\omega^{2}}{\chi_{r}^{2}}-\frac{\tau^{2}}{c^{2}}}} & 0 & 0 & \frac{\tau^{2}}{2 c^{2} \sqrt{\frac{\omega^{2}}{\chi_{r}^{2}}-\frac{\tau^{2}}{c^{2}}}\left(\frac{\omega}{\chi_{r}}-\sqrt{\frac{\omega^{2}}{\chi_{r}^{2}}-\frac{\tau^{2}}{c^{2}}}\right)} \\
0 & \frac{\mathrm{i} \tau e^{-\mathrm{i} \theta}}{2 c \sqrt{\frac{\omega^{2}}{\chi_{r}^{2}}-\frac{\tau^{2}}{c^{2}}}} & -\frac{\tau^{2}}{2 c^{2} \sqrt{\frac{\omega^{2}}{\chi_{r}^{2}}+\frac{\tau^{2}}{c^{2}}}\left(\frac{\omega}{\chi_{r}}-\sqrt{\frac{\omega^{2}}{\chi_{r}^{2}}-\frac{\tau^{2}}{c^{2}}}\right)} & 0
\end{array}\right)
$$

Now

$$
\frac{\partial \mathcal{N}_{0}}{\partial r}=\left(\begin{array}{cccc}
\alpha_{r} & 0 & \beta_{r} & 0 \\
0 & -e^{2 \mathrm{i} \theta} \beta_{r} & 0 & -e^{2 \mathrm{i} \theta} \alpha_{r} \\
0 & 0 & 0 & 0 \\
0 & 0 & 0 & 0
\end{array}\right)
$$

where

$$
\alpha_{r}=\frac{\partial \alpha}{\partial r}, \quad \beta_{r}=\frac{\partial \alpha}{\partial r}
$$

So that

$$
\mathcal{N}_{0}^{-1} \frac{\partial \mathcal{N}_{0}}{\partial r}=\left(\begin{array}{cccc}
\frac{\alpha_{r}}{\alpha-\beta} & 0 & \frac{\beta_{r}}{\alpha-\beta} & 0 \\
0 & \frac{\beta_{r}}{\beta-\alpha} & 0 & \frac{\alpha_{r}}{\beta-\alpha} \\
\frac{\alpha_{r}}{\beta-\alpha} & 0 & \frac{\beta_{r}}{\beta-\alpha} & 0 \\
0 & \frac{\beta_{r}}{\alpha-\beta} & 0 & \frac{\alpha_{r}}{\alpha-\beta}
\end{array}\right)
$$

This enables us to determine $\mathcal{R}_{0}$ then $\mathcal{K}_{-1}$. First setting

$$
\left\{\begin{aligned}
\gamma_{1} & :=-\frac{i e A_{r}-e A_{\theta}}{c} \\
\gamma_{2} & :=\frac{i e^{-\mathrm{i} \theta}\left(V_{c}+e V-m c^{2}\right)}{c} \\
\gamma_{3} & :=-\frac{i e A_{r}+e A_{\theta}}{c} \\
\gamma_{4} & :=\frac{i e^{-\mathrm{i} \theta}\left(V_{c}+e V+m c^{2}\right)}{c}
\end{aligned}\right.
$$


Then $\mathcal{N}_{0}^{-1} \mathcal{L}_{0} \mathcal{N}_{0}$ is equal to

$$
\left(\begin{array}{cccc}
\frac{\alpha \gamma_{1}+\alpha \gamma_{4}^{*} \beta+\gamma_{2}-\beta \gamma_{3}}{\alpha-\beta} & 0 & 0 & \frac{\beta \gamma_{1}+\gamma_{4}^{*} \beta^{2}+\gamma_{2}-\beta \gamma_{3}}{\alpha-\beta} \\
0 & \frac{\gamma_{3} \beta^{*} e^{-2 \mathrm{i} \theta}+\gamma_{4} \alpha \beta^{*}+\gamma_{1} \alpha-\gamma_{2}^{*} e^{-2 \mathrm{i} \theta}}{\alpha-\beta} & 0 & \frac{\alpha^{*} \gamma_{3} e^{-2 \mathrm{i} \theta}+\gamma_{4}|\alpha|^{2}+\gamma_{1} \alpha-\gamma_{2}^{*} e^{-2 \mathrm{i} \theta}}{\alpha-\beta} \\
\frac{\gamma_{1} \alpha+\gamma_{4}|\alpha|^{2}+\gamma_{3} \alpha-\gamma_{2}}{\alpha-\beta} & 0 & \frac{\gamma_{1} \beta+\alpha \beta \gamma_{4}^{*}+\gamma_{3} \alpha-\gamma_{2}}{\alpha-\beta} & 0 \\
0 & \frac{|\beta|^{2} \gamma_{4}+\beta^{*} \gamma_{3} e^{-2 \mathrm{i} \theta}+\gamma_{1} \beta-\gamma_{2}^{*} e^{-2 \mathrm{i} \theta}}{\beta-\alpha} & 0 & \frac{\alpha^{*} \beta \gamma_{4}+e^{-2 \mathrm{i} \theta} \alpha^{*} \gamma_{3}+\gamma_{1} \beta-\gamma_{2}^{*} e^{-2 \mathrm{i} \theta}}{\beta-\alpha}
\end{array}\right)
$$

We recall that:

$$
\mathcal{K}_{-1}=\frac{1}{2 \lambda}\left(\begin{array}{cccc}
0 & 0 & \left(\sigma_{0}\left(R_{0}\right)\right)_{13} & \left(\sigma_{0}\left(R_{0}\right)\right)_{14} \\
0 & 0 & \left(\sigma_{0}\left(R_{0}\right)\right)_{23} & \left(\sigma_{0}\left(R_{0}\right)\right)_{24} \\
\left(\sigma_{0}\left(R_{0}\right)\right)_{31} & \left(\sigma_{0}\left(R_{0}\right)\right)_{32} & 0 & 0 \\
\left(\sigma_{0}\left(R_{0}\right)\right)_{41} & \left(\sigma_{0}\left(R_{0}\right)\right)_{42} & 0 & 0
\end{array}\right)
$$

We now have to determine an explicit expression for $\mathcal{K}_{-1}$ in order to deduce $K_{-1}$, and to derive explicit $\mathrm{TBC} / \mathrm{ABC}$. In this goal, it is necessary to approximate $\mathcal{K}_{-1}$, to provide an expression of $K_{-1}$ involving simple (differential/pseudodifferentiel) operators. A natural approach consists of considering different kinds of regimes. Typically, we will consider high time-frequency regimes to avoid small oscillations, that is such that $\tau \gg 1$. Naturally, other frequency regimes (on $\omega \gg 1, \omega / \tau \gg 1$, «1, etc) could be considered from there. The asymptotic expansion for large $\tau$ for $\mathcal{K}_{-1}$ 's components is as follows. A simple way to achieve approximation $\mathcal{K}_{-1}$ consists of expanding $\mathcal{N}_{0}^{-1}$ and $\mathcal{N}_{0}$ for large $\tau$. First as

$$
\begin{aligned}
\alpha_{r} & =\frac{i e^{-i \theta} \tau}{\left(\omega c-\sqrt{\omega^{2} c^{2}-\tau^{2} r^{2}}\right)^{2}}\left(\omega c-\sqrt{\omega^{2} c^{2}-\tau^{2} r^{2}}-\frac{\tau^{2} r^{2}}{\sqrt{\omega^{2} c^{2}-\tau^{2} r^{2}}}\right) \\
& =\frac{i e^{-i \theta} \tau}{\left(\omega c-\sqrt{\omega^{2} c^{2}-\tau^{2} r^{2}}\right)^{2}}\left(\omega c-\frac{\omega^{2} c^{2}}{\sqrt{\omega^{2} c^{2}-\tau^{2} r^{2}}}\right)
\end{aligned}
$$

then for large $\tau, \alpha_{r}$ as well as $\beta_{r}$ are $O\left(\tau^{-1}\right)$ and $\alpha-\beta$ is $O(1)$. As a consequence $\mathcal{N}_{0}^{-1} \partial_{r} \mathcal{N}_{0}$ is a $O\left(\tau^{-1}\right)$ and has then no contribution to $\sigma_{0}\left(R_{0}\right)$ which is then equal to $\sigma_{0}\left(\mathcal{N}_{0}^{-1} \mathcal{L}_{0} \mathcal{N}_{0}\right)$.

We easily see now that

$$
\alpha-\beta=\frac{2 \mathrm{i} c e^{-\mathrm{i} \theta}}{\tau} \sqrt{\frac{\omega^{2}}{\chi_{r}^{2}}-\frac{\tau^{2}}{c^{2}}}, \quad \alpha+\beta=\frac{2 \mathrm{i} e^{-\mathrm{i} \theta} \omega c}{r \tau}
$$

and

$$
\alpha^{2}+\beta^{2}=-\frac{2 c^{2}}{\tau^{2}}\left(\frac{2 \omega^{2}}{\chi_{r}^{2}}-\frac{\tau^{2}}{c^{2}}\right)
$$

and we remark that for large $\tau$

$$
\frac{1}{\alpha-\beta}=-\frac{e^{\mathrm{i} \theta}}{2}\left(1+\frac{1 \omega^{2} c^{2}}{2} \frac{r^{2} \tau^{2}}{2}+O\left(\tau^{-4}\right)\right.
$$


and

$$
\alpha=-\frac{\tau e^{-\mathrm{i} \theta}}{c\left(\sqrt{\frac{\tau^{2}}{c^{2}}-\frac{\omega^{2}}{\chi_{r}^{2}}}+\mathrm{i} \frac{\omega}{\chi_{r}}\right)}=-e^{-\mathrm{i} \theta}\left(1-\frac{\mathrm{i} \omega c}{\tau \chi_{r}}\right)+O\left(\tau^{-2}\right)
$$

so that

$$
\frac{\alpha}{\alpha-\beta}=\frac{1}{2}\left(1-\frac{i \omega c}{\tau \chi_{r}}\right)+O\left(\tau^{-2}\right)
$$

and

$$
\frac{\beta}{\beta-\alpha}=\frac{1}{2}\left(1+\frac{i \omega c}{\tau \chi_{r}}\right)+O\left(\tau^{-2}\right)
$$

Note that

$$
\mathcal{N}_{0}^{(-1,0)}:=\left(\begin{array}{cccc}
-e^{-\mathrm{i} \theta}\left(1-\frac{\mathrm{i} \omega c}{\tau \chi_{r}}\right) & 0 & e^{-\mathrm{i} \theta}\left(1+\frac{\mathrm{i} \omega c}{\tau \chi_{r}}\right) & 0 \\
0 & -e^{\mathrm{i} \theta}\left(1+\frac{\mathrm{i} \omega c}{\tau \chi_{r}}\right) & 0 & e^{\mathrm{i} \theta}\left(1-\frac{\mathrm{i} \omega c}{\tau \chi_{r}}\right) \\
0 & 1 & 0 & 1 \\
1 & 0 & 1 & 0
\end{array}\right)
$$

and

$$
\left(\mathcal{N}_{0}^{-1}\right)^{(-1,0)}:=\left(\begin{array}{cccc}
-\frac{e^{\mathrm{i} \theta}}{2} & 0 & 0 & \frac{1}{2}\left(1+\frac{\mathrm{i} \omega c}{\tau \chi_{r}}\right) \\
0 & -\frac{e^{-\mathrm{i} \theta}}{2} & \frac{1}{2}\left(1-\frac{\mathrm{i} \omega c}{\tau \chi_{r}}\right) & 0 \\
\frac{e^{\mathrm{i} \theta}}{2} & 0 & 0 & \frac{1}{2}\left(1-\frac{\mathrm{i} \omega c}{\tau \chi_{r}}\right) \\
0 & \frac{e^{-\mathrm{i} \theta}}{2} & \frac{1}{2}\left(1+\frac{\mathrm{i} \omega c}{\tau \chi_{r}}\right) & 0
\end{array}\right)
$$

In fact, here only the order 0 terms in $\tau$, are of interest:

$$
\mathcal{N}_{0}^{-1}=\left(\mathcal{N}_{0}^{-1}\right)^{(0,0)}+O\left(\tau^{-1}\right), \quad \mathcal{N}_{0}=\mathcal{N}_{0}^{(0,0)}+O\left(\tau^{-1}\right)
$$

where

$$
\left(\mathcal{N}_{0}^{-1}\right)^{(0,0)}:=\left(\begin{array}{cccc}
-\frac{e^{\mathrm{i} \theta}}{2} & 0 & 0 & \frac{1}{2} \\
0 & -\frac{e^{-\mathrm{i} \theta}}{2} & \frac{1}{2} & 0 \\
\frac{e^{\mathrm{i} \theta}}{2} & 0 & 0 & \frac{1}{2} \\
0 & \frac{e^{-\mathrm{i} \theta}}{2} & \frac{1}{2} & 0
\end{array}\right), \mathcal{N}_{0}^{(0,0)}=\left(\begin{array}{cccc}
-e^{-\mathrm{i} \theta} & 0 & e^{-\mathrm{i} \theta} & 0 \\
0 & e^{\mathrm{i} \theta} & 0 & -e^{\mathrm{i} \theta} \\
0 & 1 & 0 & 1 \\
1 & 0 & 1 & 0
\end{array}\right)
$$


Then for large $\tau$ we have

$$
\widetilde{\sigma_{0}\left(R_{0}\right)}=-\left(\mathcal{N}_{0}^{-1}\right)^{(0,0)} \mathcal{L}_{0} \mathcal{N}_{0}^{(0,0)}-\left(\mathcal{N}_{0}^{-1}\right)^{(0,0)}\left(\frac{\partial \mathcal{N}_{0}}{\partial r}\right)^{(0,0)}
$$

In addition

$$
\frac{1}{2 \lambda}=-\frac{i c}{2 \tau}-\frac{i \omega^{2} c^{3}}{4 \tau^{3} r^{2}}+O\left(\tau^{-4}\right)
$$

So that we approximate $\mathcal{K}_{-1}$ by $\mathcal{K}_{-1}^{(-1,0)}$ defined by

$$
\mathcal{K}_{-1}^{(-1,0)}=-\frac{\mathbf{i} c}{2 \tau}\left(\begin{array}{cccc}
0 & 0 & \left(\sigma_{0}\left(R_{0}\right)\right)_{13} & \left(\sigma_{0}\left(R_{0}\right)\right)_{14} \\
0 & 0 & \left(\sigma_{0}\left(R_{0}\right)\right)_{23} & \left(\sigma_{0}\left(R_{0}\right)\right)_{24} \\
\left(\sigma_{0}\left(R_{0}\right)\right)_{31} & \left(\sigma_{0}\left(R_{0}\right)\right)_{32} & 0 & 0 \\
\left(\sigma_{0}\left(R_{0}\right)\right)_{41} & \left(\sigma_{0}\left(R_{0}\right)\right)_{42} & 0 & 0
\end{array}\right)
$$

As

$$
\left\{\begin{aligned}
\sigma_{0}\left(\mathcal{N}_{0}^{-1} \mathcal{L}_{0}\right)_{11} & =\frac{1}{c}\left(e^{\mathrm{i} \theta}\left(\mathrm{i} A_{r} e-e A_{\theta}\right)+e^{\mathrm{i} \theta} \mathrm{i}\left(V_{c}+e V+m c^{2}\right)\right) \\
\sigma_{0}\left(\mathcal{N}_{0}^{-1} \mathcal{L}_{0}\right)_{14} & =\frac{1}{c}\left(-\mathrm{i}\left(V_{c}+e V-m c^{2}\right)-\left(\mathrm{i} A_{r} e+e A_{\theta}\right)\right) \\
\sigma_{0}\left(\mathcal{N}_{0}^{-1} \mathcal{L}_{0}\right)_{22} & =\frac{1}{c}\left(\mathrm{i} e^{-\mathrm{i} \theta}\left(V_{c}+e V+m c^{2}\right)+e^{-\mathrm{i} \theta}\left(\mathrm{i} e A_{r}+e A_{\theta}\right)\right) \\
\sigma_{0}\left(\mathcal{N}_{0}^{-1} \mathcal{L}_{0}\right)_{23} & =\frac{1}{c}\left(\mathrm{i}\left(V_{c}+e V-m c^{2}\right)-\mathrm{i} A_{r} e+e A_{\theta}\right) \\
\sigma_{0}\left(\mathcal{N}_{0}^{-1} \mathcal{L}_{0}\right)_{31} & =\frac{1}{c}\left(-e^{\mathrm{i} \theta}\left(\mathrm{i} A_{r} e-e A_{\theta}\right)+e^{\mathrm{i} \theta} \mathrm{i}\left(V_{c}+e V+m c^{2}\right)\right) \\
\sigma_{0}\left(\mathcal{N}_{0}^{-1} \mathcal{L}_{0}\right)_{34} & =\frac{1}{c}\left(\mathrm{i}\left(V_{c}+e V-m c^{2}\right)-\mathrm{i} A_{r} e-e A_{\theta}\right) \\
\sigma_{0}\left(\mathcal{N}_{0}^{-1} \mathcal{L}_{0}\right)_{42} & =\frac{1}{c}\left(\mathrm{i} e^{-\mathrm{i} \theta}\left(V_{c}+e V+m c^{2}\right)-e^{-\mathrm{i} \theta}\left(\mathrm{i} e A_{r}+e A_{\theta}\right)\right) \\
\sigma_{0}\left(\mathcal{N}_{0}^{-1} \mathcal{L}_{0}\right)_{43} & =\frac{1}{c}\left(\mathrm{i}\left(V_{c}+e V-m c^{2}\right)-\mathrm{i} A_{r} e+e A_{\theta}\right)
\end{aligned}\right.
$$

Then we deduce $\sigma_{0}\left(\widetilde{\mathcal{N}_{0}^{-1} \mathcal{L}_{0}} \mathcal{N}_{0}\right)$

$\frac{2}{c}\left(\begin{array}{cccc}-i e A_{r}-\mathrm{i}\left(V_{c}+e V\right) & 0 & \mathrm{i} m c^{2}-e A_{\theta} & 0 \\ 0 & e A_{\theta}+\mathrm{i} m c^{2} & 0 & -\mathrm{i}\left(V_{c}+e V\right)-\mathrm{i} A_{r} e \\ -e A_{\theta}-\mathrm{i} m c^{2} & 0 & -\mathrm{i} e A_{r}+\mathrm{i}\left(V_{c}+e V\right) & 0 \\ 0 & \mathrm{i}\left(V_{c}+e V\right)-\mathrm{i} e A_{r} & 0 & e A_{\theta}-\mathrm{i} m c^{2}\end{array}\right)$

We can now define the operators associated to the symbols derived above. They are written as combination of classical differential and Riemann-Liouville operators. We will denote by $K_{-1}^{(-1,0)}$ the approximate operators such that $\mathcal{K}_{-1}^{(-1,0)}=\mathcal{K}_{-1}+O\left(\tau^{-2}\right)$. We recall that to any symbol $(\mathbf{i} \tau)^{-n}$ (for $n \in \mathbb{N}^{*}$ ) is associated a Riemann-Liouville operator defined by $\partial_{t}^{-n}$. 
Proposition 3.2. For large $\tau$ and neglecting the terms beyond $O\left(\tau^{-3}\right)$, Operator $K_{-1}^{(-1,0)}$ is approximated by

$$
\tilde{K}_{-1}^{(-1,0)}=\tilde{C} \partial_{t}^{-1}
$$

where

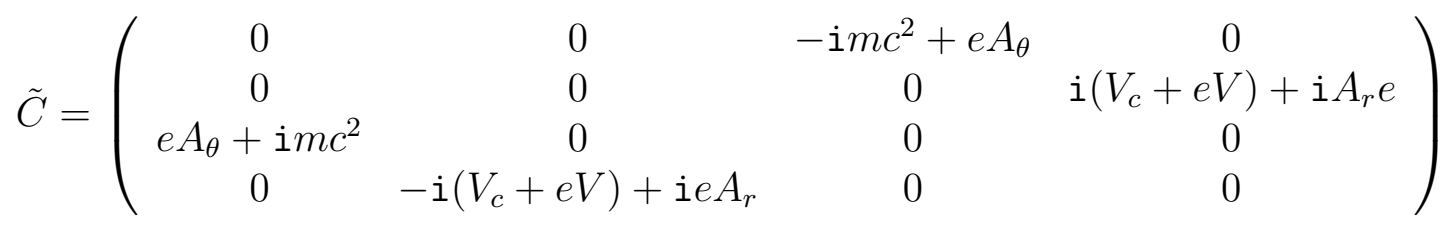

As a consequence, the outflowing part of the wave is selected by annihilating up to a certain order, the part of $\mathbf{w}_{-m}$ which propagates in the opposite direction of the outward normal vector to the boundary [8].

Proposition 3.3. The $A B C$ at order 0 and 1 (corresponding to vanish the outgoing waves from the small crown surrounding $\Omega_{R}$ ) are defined as follows. Setting $\mathbf{w}_{0}=V_{0}^{-1} \mathbf{u}$ and $\mathbf{w}_{-1}=\left(I+K_{-1}\right) \mathbf{w}_{0}$, the $A B C$ write

- At order 0: $\left(V_{0}^{-1} \mathbf{u}\right)_{1,2}=\left(\mathbf{w}_{0}\right)_{1,2}=\mathbf{0}$ on $\Sigma^{R}$

- At order 1: $\left(V_{-1} \mathbf{u}\right)_{1,2}=\left(\mathbf{w}_{-1}\right)_{1,2}=\mathbf{0}$ on $\Sigma^{R}$, where $V_{-1}=\left(I+K_{-1}\right) V_{0}^{-1}$.

This simply consists of vanishing the incoming waves (inside $\Omega_{R}$ ).

No additional condition (beyond the TDDE itself) on outgoing waves is required. In order to actually determine an explicit $\mathrm{ABC},\left(\left(I+K_{-1}\right) V_{0}^{-1} \mathbf{u}\right)_{1,2}=\mathbf{0}$, it is necessary to evaluate $V_{0}^{-1}$ and $K_{-1} V_{0}^{-1}$. In this goal, and as above we have to expand $\mathcal{N}_{0}^{-1}$ and $\mathcal{K}_{-1} \mathcal{N}_{0}^{-1}$ for $\tau$ large using (29) and an asymptotic expansion. From the above study, we deduce that:

$$
\left(V_{0}^{-1}\right)^{(0,0)}=\left(\begin{array}{cccc}
-\frac{e^{\mathrm{i} \theta}}{2} & 0 & 0 & \frac{1}{2} \\
0 & -\frac{e^{-\mathrm{i} \theta}}{2} & \frac{1}{2} & 0 \\
\frac{e^{\mathrm{i} \theta}}{2} & 0 & 0 & \frac{1}{2} \\
0 & \frac{e^{\mathrm{i} \theta}}{2} & \frac{1}{2} & 0
\end{array}\right), V_{0}^{(0,0)}=\left(\begin{array}{cccc}
-e^{-\mathrm{i} \theta} & 0 & e^{-\mathrm{i} \theta} & 0 \\
0 & -e^{\mathrm{i} \theta} & 0 & e^{\mathrm{i} \theta} \\
0 & 1 & 0 & 1 \\
1 & 0 & 1 & 0
\end{array}\right)
$$

We also have

$$
\left(V_{0}^{-1}\right)^{(-1,0)}=\left(\begin{array}{cccc}
-\frac{e^{\mathrm{i} \theta}}{2} & 0 & 0 & \frac{1}{2}\left(1+\frac{\mathrm{i} c}{\chi_{r}} \partial_{\theta} \partial_{t}^{-1}\right) \\
0 & -\frac{e^{-\mathrm{i} \theta}}{2} & \frac{1}{2}\left(1-\frac{\mathrm{i} c}{\chi_{r}} \partial_{\theta} \partial_{t}^{-1}\right) & 0 \\
\frac{e^{\mathrm{i} \theta}}{2} & 0 & 0 & \frac{1}{2}\left(1-\frac{\mathrm{i} c}{\chi_{r}} \partial_{\theta} \partial_{t}^{-1}\right) \\
0 & \frac{e^{i \theta}}{2} & \frac{1}{2}\left(1+\frac{i c}{\chi_{r}} \partial_{\theta} \partial_{t}^{-1}\right) & 0
\end{array}\right)
$$


and

$$
V_{0}^{(-1,0)}=\left(\begin{array}{cccc}
-e^{-i \theta}\left(1-\frac{\mathrm{i} c}{\chi_{r}} \partial_{\theta} \partial_{t}^{-1}\right) & 0 & e^{-\mathrm{i} \theta}\left(1+\frac{\mathrm{i} c}{\chi_{r}} \partial_{\theta} \partial_{t}^{-1}\right) & 0 \\
0 & -e^{\mathrm{i} \theta}\left(1+\frac{\mathrm{i} c}{\chi_{r}} \partial_{\theta} \partial_{t}^{-1}\right) & 0 & e^{\mathrm{i} \theta}\left(1-\frac{\mathrm{i} c}{\chi_{r}} \partial_{\theta} \partial_{t}^{-1}\right) \\
0 & 1 & 0 & 1 \\
1 & 0 & 1 & 0
\end{array}\right)
$$

Now using, $\left(K_{-1}\right)^{(-1,0)}$ and $\left(V_{0}\right)^{(-1,0)}$, we get an explicit expression of $V_{-1}^{(-1,0)}$ which is:

$$
V_{-1}^{(-1,0)}=\frac{1}{2}\left(\begin{array}{ll}
A^{(-1,0)} & B^{(-1,0)} \\
C^{(-1,0)} & D^{(-1,0)}
\end{array}\right)
$$

with

$$
\begin{aligned}
& A_{-1}^{(-1,0)}=\frac{1}{2}\left(\begin{array}{cc}
\left(-\left(\mathbf{i} m c^{2}-e A_{\theta}\right) \partial_{t}^{-1}-1\right) e^{\mathrm{i} \theta} & 0 \\
0 & \left(\left(\mathrm{i}\left(V_{c}+e V\right)+\mathrm{i} e A_{r} e\right) \partial_{t}^{-1}-1\right) e^{-\mathrm{i} \theta}
\end{array}\right) \\
& B_{-1}^{(-1,0)}=\frac{1}{2}\left(\begin{array}{cc}
0 & -\left(\mathbf{i} m c^{2}-e A_{\theta}\right) \partial_{t}^{-1}+1 \\
\left(\mathbf{i}\left(V_{c}+e V\right)+\mathbf{i} e A_{r} e\right) \partial_{t}^{-1}+1 & 0
\end{array}\right) \\
& C_{-1}^{(-1,0)}=\frac{1}{2}\left(\begin{array}{cc}
\left(1-\left(e A_{\theta}+\mathrm{i} m c^{2}\right) \partial_{t}^{-1}\right) e^{\mathrm{i} \theta} & 0 \\
0 & \left(1+\left(\mathbf{i}\left(V_{c}+e V\right)-\mathrm{i} e A_{r}\right) \partial_{t}^{-1}\right) e^{-\mathbf{i} \theta}
\end{array}\right) \\
& D_{-1}^{(-1,0)}=\frac{1}{2}\left(\begin{array}{cc}
0 & 1+\left(e A_{\theta}+\mathbf{i} m c^{2}\right) \partial_{t}^{-1} \\
1-\left(\mathrm{i}\left(V_{c}+e V\right)-\mathrm{i} e A_{r}\right) \partial_{t}^{-1} & 0
\end{array}\right)
\end{aligned}
$$

The process continues with higher order ABC but are not computed here. The discretization of this condition will be discussed in Section 5. The ABC which are derived so far depends on the 4 components of the unknown and are independent of the derivative with respect to $r$. The ABC, $\left(V_{0} \mathbf{u}\right)_{1,2}=\mathbf{0}$, then $\left.\left(V_{0}+K_{-1} V_{0}\right) \mathbf{u}\right)_{1,2}=\mathbf{0}$ are easily analytically evaluated.

\subsection{Application to 1-d Dirac equation}

A direct application to the above conditions in a 1-d framework simply necessitates to vanish angular derivatives and set $\theta$ to 0 . The domain is denoted by $(-a, a)$ and the equation simply writes

$$
\left(\partial_{r}+L\left(r, \theta, t, \partial_{t}\right)\right) \mathbf{u}=\mathbf{0}
$$

where

$$
L_{1}=\frac{1}{c} \tilde{\alpha}_{x} \partial_{t} L_{0}=\frac{1}{c} \tilde{\alpha}_{x} \tilde{\beta}
$$




$$
\tilde{\alpha}_{x}=\left(\begin{array}{cccc}
0 & 0 & 0 & 1 \\
0 & 0 & 1 & 0 \\
0 & 1 & 0 & 0 \\
1 & 0 & 0 & 0
\end{array}\right)
$$

and

$$
\tilde{\beta}=i \beta m c^{2}+i\left(e V+V_{c}\right) \mathbf{I}_{4}-i e A_{x} \alpha_{x}
$$

that is

$$
\tilde{\beta}=\left(\begin{array}{cccc}
i\left(V_{c}+e V+m c^{2}\right) & 0 & 0 & -i e A_{x} \\
0 & i\left(V_{c}+e V+m c^{2}\right) & -i e A_{x} & 0 \\
0 & -i e A_{x} & i\left(V_{c}+e V-m c^{2}\right) & 0 \\
-i e A_{x} & 0 & 0 & i\left(V_{c}+e V-m c^{2}\right)
\end{array}\right)
$$

and $\tilde{\alpha}_{x} \tilde{\beta}=$

$$
\left(\begin{array}{cccc}
-i e A_{x} & 0 & 0 & i\left(V_{c}+e V-m c^{2}\right) \\
0 & -i e A_{x} & i\left(V_{c}+e V-m c^{2}\right) & 0 \\
0 & i\left(V_{c}+e V+m c^{2}\right) & -i e A_{x} & 0 \\
i\left(V_{c}+e V+m c^{2}\right) & 0 & 0 & -i e A_{x} .
\end{array}\right)
$$

We deduce that the TDDE in 1-d writes

$$
\left\{\begin{array}{l}
c \partial_{x} u_{1}+\partial_{t} u_{4}-i e A_{x} u_{1}+i\left(V_{c}+e V-m c^{2}\right) u_{4}=0 \\
c \partial_{x} u_{2}+\partial_{t} u_{3}-i e A_{x} u_{2}+i\left(V_{c}+e V-m c^{2}\right) u_{3}=0 \\
c \partial_{x} u_{3}+\partial_{t} u_{2}+i\left(V_{c}+e V+m c^{2}\right) u_{2}-i e A_{x} u_{3}=0 \\
c \partial_{x} u_{4}+\partial_{t} u_{1}+i\left(V_{c}+e V+m c^{2}\right) u_{1}-i e A_{x} u_{4}=0
\end{array}\right.
$$

As

$$
V_{0}^{(-1,0)}=\left(\begin{array}{cccc}
-1 & 0 & 1 & 0 \\
0 & -1 & 0 & 1 \\
0 & 1 & 0 & 1 \\
1 & 0 & 1 & 0
\end{array}\right),\left(V_{0}^{-1}\right)^{(-1,0)}=\frac{1}{2}\left(\begin{array}{cccc}
-1 & 0 & 0 & 1 \\
0 & -1 & 1 & 0 \\
1 & 0 & 0 & 1 \\
0 & 1 & 1 & 0
\end{array}\right)
$$

The first right (resp. left) $\mathrm{ABC}$ writes:

$$
u_{1}-u_{4}=0, u_{2}-u_{3}=0,\left(\text { resp. } u_{1}+u_{4}=0, u_{2}+u_{3}=0\right)
$$

Similarly we derive explicit $\mathrm{ABC}$ from $\left(V_{0}^{-1}+K_{-1} V_{0}^{-1}\right)_{1,2} \mathbf{u}=\mathbf{0}$ with $V_{-1}^{(-1,0)}$ equal to

$$
\frac{1}{2}\left(\begin{array}{cccc}
-i m c^{2} \partial_{t}^{-1}-1 & 0 & 0 & 1-i m c^{2} \partial_{t}^{-1} \\
0 & \left(i\left(V_{c}+e V\right)+i e A_{x} e\right) \partial_{t}^{-1}-1 & \left(i\left(V_{c}+e V\right)+i e A_{x} e\right) \partial_{t}^{-1}+1 & 0 \\
1-i m c^{2} \partial_{t}^{-1} & 0 & 0 & 1+i m c^{2} \partial_{t}^{-1} \\
0 & 1+\left(i\left(V_{c}+e V\right)-i e A_{x}\right) \partial_{t}^{-1} & 1-\left(i\left(V_{c}+e V\right)-i e A_{x}\right) \partial_{t}^{-1} & 0 .
\end{array}\right)
$$

The second $\mathrm{ABC}$ writes:

$$
\left(-\mathrm{i} m c^{2} \partial_{t}^{-1}-1\right) u_{1}+\left(-\mathrm{i} m c^{2} \partial_{t}^{-1}+1\right) u_{4}=0
$$

and

$$
\left(\left(i\left(V_{c}+e V\right)+i e A_{x}\right) \partial_{t}^{-1}-1\right) u_{2}+\left(\left(i\left(V_{c}+e V\right)+i e A_{x} e\right) \partial_{t}^{-1}+1\right) u_{3}=0
$$




\section{Other techniques}

We now present some alternative techniques to the derivation of ABC for TDDE or TDKGE.

\subsection{Volkov approach}

We here give some elements of derivation of ABC based on the Volkov wavefunction in 3-d. The principle is based on the fact that the TDDE have explicit solutions called Volkov wavefunctions, when the Coulomb potential is set zero. The approach is very similar to the one proposed in [24], for laser-molecule time dependent Schrödinger equations (TDSE). Roughly speaking the idea is as follows. We first rewrite the TDDE in the form

$$
\left\{\begin{array}{l}
\partial_{t} \mathbf{u}=\left(P_{1}+P_{2}\right) \mathbf{u} \text { on } \mathbb{R}^{3} \times[0, T] \\
\mathbf{u}(\cdot, 0)=\mathbf{u}_{i}(\cdot) \text { on } \mathbb{R}^{2}
\end{array}\right.
$$

where in $3-\mathrm{d}$

$P_{1}=-\alpha_{x}\left(c \partial_{x}-\mathrm{i} e A_{x}\right)-\alpha_{y}\left(c \partial_{y}-\mathrm{i} e A_{y}\right)-\alpha_{z}\left(c \partial_{z}-\mathrm{i} e A_{z}\right)-\mathrm{i} \beta m c^{2}, P_{2}=-\left(\mathrm{i} V_{c}+e V\right) \mathbf{I}_{4}$

and

$$
\alpha_{z}=\left(\begin{array}{cc}
0_{2} & \sigma_{z} \\
\sigma_{z} & 0_{2}
\end{array}\right)
$$

with

$$
\sigma_{z}=\left(\begin{array}{cc}
0 & -1 \\
1 & 0
\end{array}\right)
$$

We introduce a fictitious domain $\Omega$ such that $\operatorname{supp}\left(\mathbf{u}_{0}\right) \subseteq \Omega$ and $\operatorname{supp}\left(V_{c}\right) \subseteq \Omega$. As usual, we determine an operator $\mathcal{B}$

$$
\left\{\begin{array}{l}
\partial_{t} \tilde{\mathbf{u}}=\left(P_{1}+P_{2}\right) \tilde{\mathbf{u}} \text { on } \Omega \times[0, T] \\
\tilde{\mathbf{u}}(\cdot, 0)=\mathbf{u}_{0}(\cdot) \text { on } \Omega \\
\mathcal{B} \cdot \tilde{\mathbf{u}}=0 \text { on } \partial \Omega \times[0, T]
\end{array}\right.
$$

such that $\mathbf{u}_{\mid \Omega} \sim \tilde{\mathbf{u}}$. We remark that in $\Omega^{c} \subseteq \mathbb{R}^{3}$, the Dirac equation writes

$$
\partial_{t} \mathbf{u}=P_{1} \mathbf{u}
$$

It turns out that this equation admits an exact solution in $\mathbb{R}^{3}$, see [25], that we will call $\mathbf{u}_{v}$. Denoting and assuming that 
- the incoming wave is a plane electromagnetic wave, that is $V=0$. Its amplitude is denoted by $\mathbf{A}_{0}$.

- $\mathbf{A}:=\left(A_{x}, A_{y}, A_{z}\right)^{T}$ is supposed to be in the form $\mathbf{A}(\mathbf{x}, t)=\mathbf{A}_{0} f(\omega t-\mathbf{k} \cdot \mathbf{r})$, with $\mathbf{x}=(x, y, z), f$ is a given function, $\omega$ (resp. $\mathbf{k}$ ) the imposed incoming pulse frequency (resp. 3-d photon momentum).

- $A:=\left(0, \mathbf{A}^{T}\right), k:=(\omega, \mathbf{k}), \mathbf{n}:=\mathbf{k} /\|\mathbf{k}\|, \mathbf{e}:=\mathbf{A} /\|\mathbf{A}\|^{2}$ and $\alpha=\left(\alpha_{x}, \alpha_{y}, \alpha_{z}\right)$.

Volkov wavefunction $\mathbf{u}_{v}$ is searched in the form

$$
\mathbf{u}_{v}(\mathbf{x}, t)=e^{-\mathbf{i}\left(p_{0} t-\mathbf{p} \cdot \mathbf{x}\right)} \phi(\tau)
$$

where $p:=\left(p_{0}, \mathbf{p}\right)$ is a constant four-component vector chosen such that $p_{0}^{2}-\|\mathbf{p}\|^{2}=m^{2}$ and $\tau=t-\frac{\mathbf{k} \cdot \mathbf{r}}{\omega}$. Now, according to $[25]$ :

Proposition 4.1. For c set to 1 and for $\mathbf{c}$ an arbitrary constant bispinor, a solution to (35) is given by

$$
\mathbf{u}_{v}(\mathbf{x}, t)=\left[1+\frac{e \alpha_{s}}{2(p \cdot k)} A\right] \frac{\mathbf{c}}{\sqrt{2 p_{0}}} e^{i S(\tau)}
$$

where the phase function is given by

$$
S(\tau)=-p_{0} t+\mathbf{p} \cdot \mathbf{x}+\int^{\tau}\left[\frac{e(\mathbf{p} \cdot \mathbf{A})}{p \cdot k}-\frac{e^{2}\|\mathbf{A}\|^{2}}{2(p \cdot k)}\right] d \tau, \quad \tau=t-\frac{\mathbf{k} \cdot \mathbf{r}}{\omega}
$$

with

$$
\alpha_{s}=\alpha_{x}+\alpha_{y} e_{y}+\alpha_{z} e_{z}+\frac{k}{2 \omega}(\alpha \times \alpha) \cdot(\mathbf{n} \times \mathbf{e}), \quad p \cdot k=p_{0} \omega-\mathbf{p} \cdot \mathbf{k}
$$

In fine, we should sum this solution over all the momenta $\left(p_{0}, \mathbf{p}\right)$ which naturally leads a huge computational complexity. An important effort should be done on developing efficient techniques to evaluate this Volkov wavefunction for the laser-particle TDDE. Note that, it is naturally possible to select certain frequency regimes in order to reduce the computational cost due to the overall sum.

Beyond, the computational difficulty to numerically evaluate this function, it is unfortunately not possible to directly impose $\mathbf{u}_{v}$ as boundary condition, due to the fact this vectorial function is defined as an integral in $\mathbb{R}^{3}$. As a consequence to be valid, the Coulomb potential have to be zero everywhere. In that case $\left(V_{c} \equiv 0\right)$, imposing

$$
\mathcal{B} \cdot \mathbf{u}=\mathbf{u}_{v} \text { on } \partial \Omega
$$

would constitute a TBC. In the general case $\left(V_{c} \neq 0\right)$ it is then necessary to include the Coulomb potential in the expression of $\mathbf{u}_{v}$, to obtain a relevant ABC. A natural idea consists 
of splitting the equation as follows. Assuming that the solution is known at time $t_{n}$, and for some small $\Delta t_{n}>0$, the solution to

$$
\begin{cases}\partial_{t} \tilde{\mathbf{u}} & =\left(P_{1}+P_{2}\right) \tilde{\mathbf{u}} \text { for }\left(t_{n}, t_{n}+\Delta t_{n}\right] \\ \tilde{\mathbf{u}}\left(\cdot, t_{n}\right) & =\tilde{\mathbf{u}}_{0}(\cdot)\end{cases}
$$

can be approximated, using the Trotter-Kato formula [9], by the solution of

$$
\left\{\begin{array}{l}
\partial_{t} \tilde{\mathbf{u}}=P_{1} \tilde{\mathbf{u}} \text { for }\left(t_{n}, t_{n}+\Delta t_{n}\right] \\
\tilde{\mathbf{u}}\left(\cdot, t_{n}\right)=\tilde{\mathbf{u}}_{n}(\cdot) \\
\partial_{t} \tilde{\mathbf{u}}=P_{2} \tilde{\mathbf{u}} \text { for }\left(t_{n}, t_{n}+\Delta t_{n}\right] \\
\tilde{\mathbf{u}}\left(\cdot, t_{n}\right)=e^{\Delta t_{n} P_{1}} \tilde{\mathbf{u}}_{n}(\cdot)
\end{array}\right.
$$

In the appropriate Hilbert space $H$, we can prove that $\left\|e^{\Delta t_{n}\left(P_{1}+P_{2}\right)} \tilde{\mathbf{u}}\left(\cdot, t_{n}\right)-e^{\Delta t_{n} P_{1}} e^{\left.\Delta t_{n} P_{2}\right)} \tilde{\mathbf{u}}\left(\cdot, t_{n}\right)\right\|_{H}$ is a $\mathcal{O}\left(\Delta t_{n}^{2}\left[P_{1}, P_{2}\right]\right)$. This gives an approximate Volkov-Coulomb wavefunction for TDDE denoted $\mathbf{u}_{v c}^{n+1}$. As a consequence, the new mixed problem consists of solving, for all $n$ such that $\sum_{i=1}^{n} \Delta t_{i} \leqslant T$,

$$
\begin{cases}\partial_{t} \tilde{\mathbf{u}} & =\left(P_{1}+P_{2}\right) \tilde{\mathbf{u}} \text { on } \Omega \times\left(t_{n}, t_{n+1}\right] \\ \tilde{\mathbf{u}}\left(\cdot, t_{n}\right) & =\mathbf{u}^{n}(\cdot) \text { on } \Omega \\ \tilde{\mathbf{u}} & =\mathbf{u}_{v c}^{n} \text { on } \partial \Omega \times\left(t_{n}, t_{n+1}\right]\end{cases}
$$

where at time $t_{n}$, the numerical solution is denoted $\mathbf{u}^{n+1}$ and $\mathbf{u}_{v c}^{n+1}$ is solution to (37). Higher order ABC are naturally possible. The main interest of this approach comes from the fact that both

$$
\partial_{t} \tilde{\mathbf{u}}=P_{1} \tilde{\mathbf{u}}, \quad \partial_{t} \tilde{\mathbf{u}}=P_{2} \tilde{\mathbf{u}}
$$

can be solved "analytically" (the equation system is a trivial diagonal system). As a consequence, from the analytical point of view, it is possible to derive very accurate ABC (simply by increasing the order of the operator splitting). However, we face a similar problem as the one for TDSE. To have a small error, $\Delta t_{n}$ has to be chosen small. The issue comes from the fact that the solution to $\partial_{t} \tilde{\mathbf{u}}=P_{1} \tilde{\mathbf{u}}$ in (37) possesses an oscillatory spatial integral of frequency $1 / \Delta t_{n}$. As a consequence, a fine numerical computation of this integral, requiring $\Delta t_{n} \ll 1$, can be computationally costly. The approach which was proposed in [24] for TDSE is still applicable here. This consists of using Filon's approximation of highly oscillatory integrals (typically the convergence is a positive power of the time step [19], [20]). This approach for TDDE, will be implemented and studied in a forthcoming paper, devoted to accurate numerical discretization of ABC for TDDE and TDKGE. 


\subsection{Basic transformations on TDKGE and TDDE}

In the last 2 sections we have derived ABC for TDKGE and TDDE using two distinct but close techniques. This section is devoted to some simple transformations on TDKGE. We show that these transformations allow to rewrite these equations in such form that existing techniques can (almost directly) be applied to derive ABC. The presentation will be laser-free, although most of these ideas are applicable with laser-particle equations.

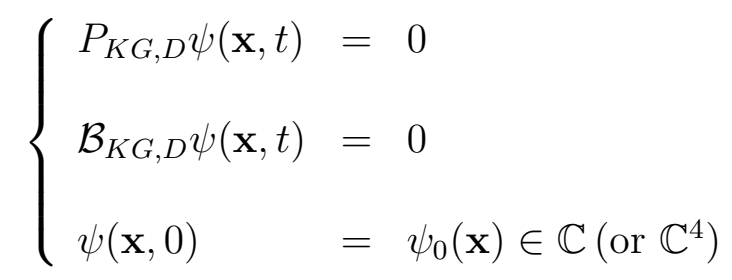

with $\mathbf{x}=(x, y)$ and

$$
\left\{\begin{array}{l}
P_{K G}=c^{2}\left(\partial_{x}^{2}+\partial_{y}^{2}+\partial_{z}^{2}\right)-\partial_{t}^{2}-m^{2} c^{4} \\
P_{D}=i \mathbf{I}_{4} \partial_{t}+\mathbf{i} c \alpha_{x} \partial_{x}+\mathbf{i} c \alpha_{y} \partial_{y}+\mathbf{i} c \alpha_{z} \partial_{z}-\beta m c^{2} .
\end{array}\right.
$$

- TDKGE to reaction-diffusion equation. We set

$$
\chi:=\left(\begin{array}{c}
\chi_{1} \\
\chi_{2}
\end{array}\right)=\left(\begin{array}{c}
\varphi \\
\partial_{t} \varphi
\end{array}\right) .
$$

In that case, it is easy to show that $\chi$ satisfies the following equation

$$
\partial_{t} \chi=A\left(\partial_{x}^{2}+\partial_{y}^{2}+\partial_{z}^{2}\right) \chi+B \chi
$$

where

$$
A=\left(\begin{array}{cc}
0 & 0 \\
c^{2} & 0
\end{array}\right), \quad B=\left(\begin{array}{cc}
0 & 1 \\
-m^{2} c^{4} & 0
\end{array}\right) .
$$

Reaction-diffusion problems are naturally very studied in the literature (in chemistry, fluid dynamics, etc) in particular from a boundary condition point of view.

- TDKGE to TDDE. There exists a fundamental connection between field-free TDKGE and TDDE. Indeed, we easily check that

$$
\begin{aligned}
\left(\partial_{x}^{2}+\partial_{y}^{2}+\partial_{z}^{2}-\frac{1}{c^{2}} \partial_{t}^{2}+m^{2} c^{4}\right) \mathbf{I}_{4}= & \left(\alpha_{x} \partial_{x}+\alpha_{y} \partial_{y}+\alpha_{z} \partial_{z}+\frac{i}{c} \mathbf{I}_{4} \partial_{t}+m c^{2} \beta\right) \\
& x\left(\alpha_{x} \partial_{x}+\alpha_{y} \partial_{y}+\alpha_{z} \partial_{z}+\frac{\dot{i}}{c} \mathbf{I}_{4} \partial_{t}+m c^{2} \beta\right) .
\end{aligned}
$$

As a consequence, the derivation of TBC/ABC from TDKGE (resp. TDDE) can be useful to derive TBC/ABC to TDDE (resp. TDKGE). We roughly describe a possible 
approach. Assuming that ABC have been derived for TDDE. The factorization (38), consists of writing TDKGE as

$$
\mathbf{I}_{4} P_{K G}=P_{D} P_{D}^{*}
$$

Now, we have seen that $P_{D}$ can be block-diagonalized, which formally writes

$$
P_{D}=V^{-1}\left(\mathbf{I}_{4} \partial_{r}-\Lambda\right) V+R
$$

where the transition operator $V$ can be explicitly constructed, and $R \in O P S^{-\infty}$. Similarly, we have seen that $P_{K G}$ can be factorized as follows

$$
\mathbf{I}_{4} P_{K G}=\mathbf{I}_{4}\left(\partial_{r}+\Lambda^{+}\right)\left(\partial_{r}-\Lambda^{-}\right)+R .
$$

As a consequence we can formally state that

$$
\left(\partial_{r}+\Lambda^{+}\right)\left(\partial_{r}-\Lambda^{-}\right) \mathbf{I}_{4}=\left(V^{-1}\left(\mathbf{I}_{4} \partial_{r}-\Lambda\right) V\right)\left(V^{-1}\left(\mathbf{I}_{4} \partial_{r}-\Lambda\right) V\right)^{*}+R
$$

where $\Lambda$ and $\Lambda^{ \pm}$are first order operators and $V$ and an operator of leading order 0 . From there it is possible to find connections between these 3 operators. We do not go further in this direction, but we think that this could be an interesting question to study.

\section{Numerical Simulations}

This section is devoted to some basic 1-d illustrations of the boundary conditions derived above. More advanced numerical simulations, as well as derivation of accurate discretization of 2-d boundary conditions and analysis of the overall schemes will be presented in a forthcoming paper.

\subsection{Discretization for TDKGE}

The discretizations which are proposed in this paper are relatively naive but still accurate. We respectivelly denote by $\Delta x$ the space step and $\Delta t_{n}$ time step at time $t_{n}=\sum_{i=1}^{n}$. As usual $\varphi_{j}^{n}$ denotes an approximation of the exact solution $\phi$, in $\left(j \Delta x, t_{n}\right)$, for $j \in \mathbb{Z}$ and $n \in \mathbb{N}^{*}$. The initial data is a wavepacket defined by

$$
\varphi_{0}(x)=\frac{c k_{0}}{m c^{2}+\sqrt{m^{2} c^{4}+c^{2} k_{0}^{2}}} \exp \left(-\frac{1}{4 x^{2}}+\mathrm{i} k_{0} x\right)
$$

where the wavenumber $k_{0}=5$. In the following, we impose $\hbar=c=m=e=1$.

\section{Case 1}

We first consider the laser-free Klein-Gordon equation on a bounded domain $[-a, a]$, approximated by an explicit scheme:

$$
\varphi_{j}^{n+1}-2 \varphi_{j}^{n}+\varphi_{j}^{n-1}=\Delta t^{2}\left[\frac{c^{2}}{\Delta x^{2}}\left(\varphi_{j+1}^{n}-2 \varphi_{j}^{n}+\varphi_{j-1}^{n}\right)-m^{2} c^{4} \varphi_{j}^{n}\right] .
$$


As seen above, an $\mathrm{ABC}$ at $+a$ is given by

$$
c \partial_{x} \varphi(x, t)+\partial_{t} \varphi(x, t)+\frac{m^{2} c^{4}}{2} \int_{0}^{t} \varphi(x, s) d s=0
$$

which is an improvement of the simple condition:

$$
\left(\partial_{t}+c \partial_{x}\right) \varphi(x, t)=0 .
$$

Above and in the following $j \in\{1, \cdots, N\}$, with $x_{1}=-a$ and $x_{N}=+a$. We numerically compare the following conditions:

- Dirichlet: $\varphi( \pm a, t)=0$, for all $t$;

- transport (40) (whose symbol includes $\lambda_{1}^{+}$and $\lambda_{0}^{+}$);

- improved transport (39) (whose symbol includes $\lambda_{1}^{+}, \lambda_{0}^{+}$and $\lambda_{-1}^{+}$);

We represent, as usual, the quantity $(t, x, \log |u(x, t)|)$ which enlights the reflections at the domain boundary. In the tests we choose $\Delta x=\Delta t_{n}=\Delta t=0.04$ for all $n, T=18$ and $a=10$. At the left boundary is implemented Dirichlet's boundary condition. At the right boundary, we implement the transport condition Fig. 1, and improved transport Fig. 2.

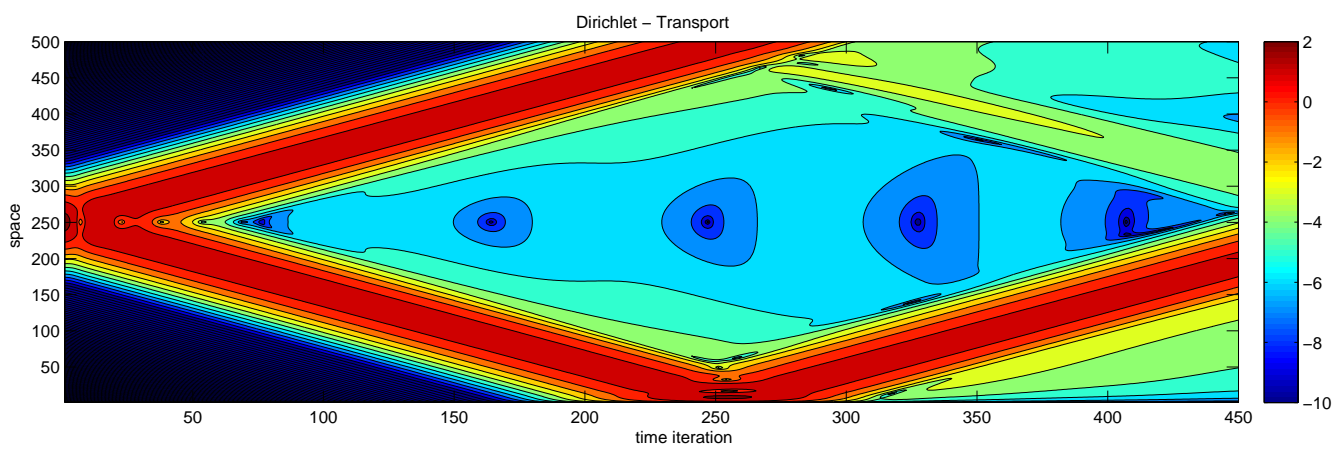

Figure 1: Klein-Gordon with Dirichlet (left) and transport (right) boundary conditions

As expected, the improved transport condition (39) derived in this paper is better than the 2 other implemented ones ((40) and Dirichlet).

\section{Case 2}

In the second example, we consider a laser-particle Klein-Gordon equation. This time, the scheme (and the derived $\mathrm{ABC}$ ) is more complex and writes

$$
\begin{aligned}
\varphi_{j}^{n+1}-2 \varphi_{j}^{n}+\varphi_{j}^{n-1}= & \Delta t^{2}\left[\frac{c^{2}}{\Delta x^{2}}\left(\varphi_{j+1}^{n}-2 \varphi_{j}^{n}+\varphi_{j-1}^{n}\right)+\frac{A_{j}^{n}}{2 \Delta t}\left(\varphi_{j}^{n+1}-\varphi_{j}^{n-1}\right)\right. \\
& \left.+\frac{B_{j}^{n}}{2 \Delta x}\left(\varphi_{j+1}^{n}-\varphi_{j-1}^{n}\right)+C_{j}^{n} \varphi_{j}^{n}\right]
\end{aligned}
$$




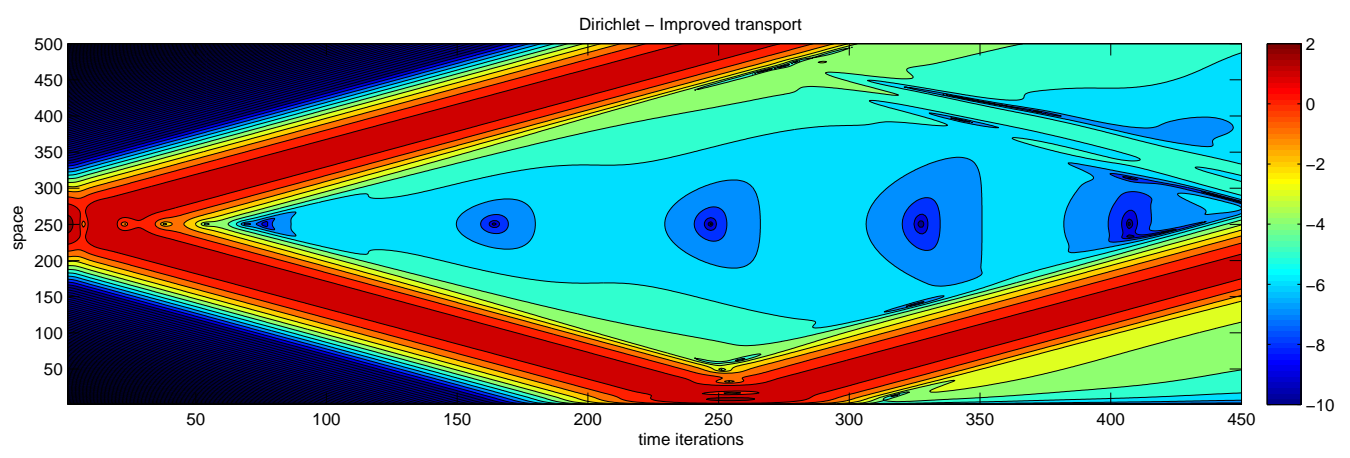

Figure 2: Klein-Gordon with Dirichlet (left) and improved transport (right) boundary conditions

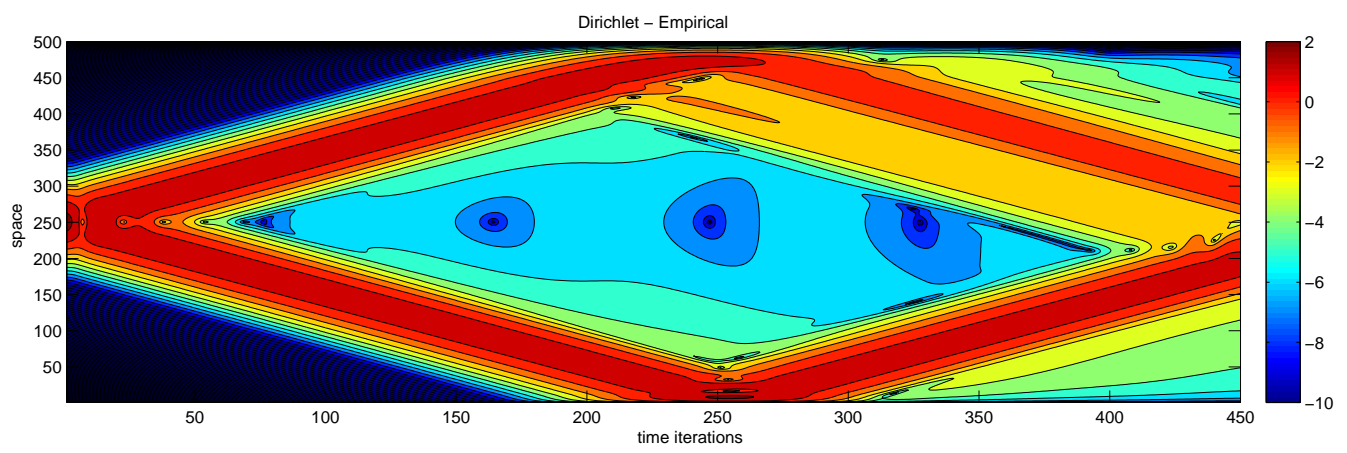

Figure 3: Klein-Gordon with Dirichlet (left) and empirical (right) boundary conditions

where

$$
\left\{\begin{array}{l}
A_{j}^{n}=-i e V\left(x_{j}, t_{n}\right) \\
B_{j}^{n}=-i e A_{x}\left(x_{j}, t_{n}\right) \\
C_{j}^{n}=-i e \partial_{t} V\left(x_{j}, t_{n}\right)-i c^{2} \partial_{x} A_{x}\left(x_{j}, t_{n}\right)+e^{2} V^{2}\left(x_{j}, t_{n}\right)-c^{2} e^{2} A_{x}^{2}\left(x_{j}, t_{n}\right)-m^{2} c^{4}
\end{array}\right.
$$

with boundary conditions imposed at $x= \pm a$, according to (18) and (20). At $-a$, we impose a Dirichlet boundary, and at $+a$, we impose i) a transport condition (operator symbol includes only $\lambda_{1}^{+}$) Fig. 4, ii) a transport condition including an order 0 operator (operator symbol includes $\lambda_{1}^{+}$and $\lambda_{0}^{+}$) Fig. 5 iii) a transport condition including an order 0 and order -1 operators (operator symbol includes $\lambda_{1}^{+}, \lambda_{0}^{+}$and $\lambda_{-1}^{+}$) Fig. 6. We assume that $A(t)=A_{0} \cos (\omega t), V(t)=E_{0} \sin (\omega t)$ with $A_{0}=1, E_{0}=0.1$, and $\omega=1$. Again $a=10$, $\Delta x=\Delta t=0.4$.

As expected the absorption is improved by including additional operators in the ABC.

Stability for the interior scheme: laser-free-TDKGE. The stability analysis for the 


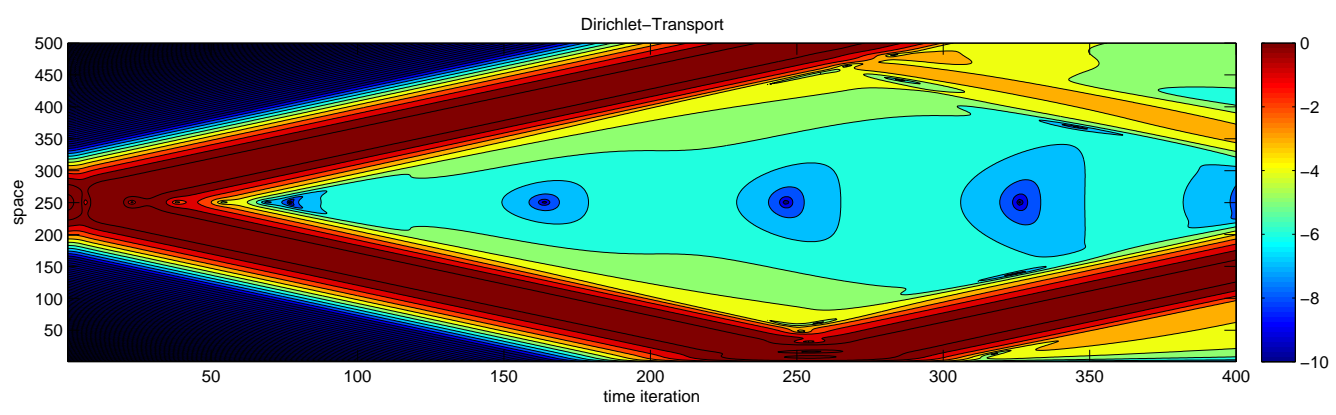

Figure 4: Laser-Klein-Gordon with Dirichlet (left) and transport (right) boundary conditions

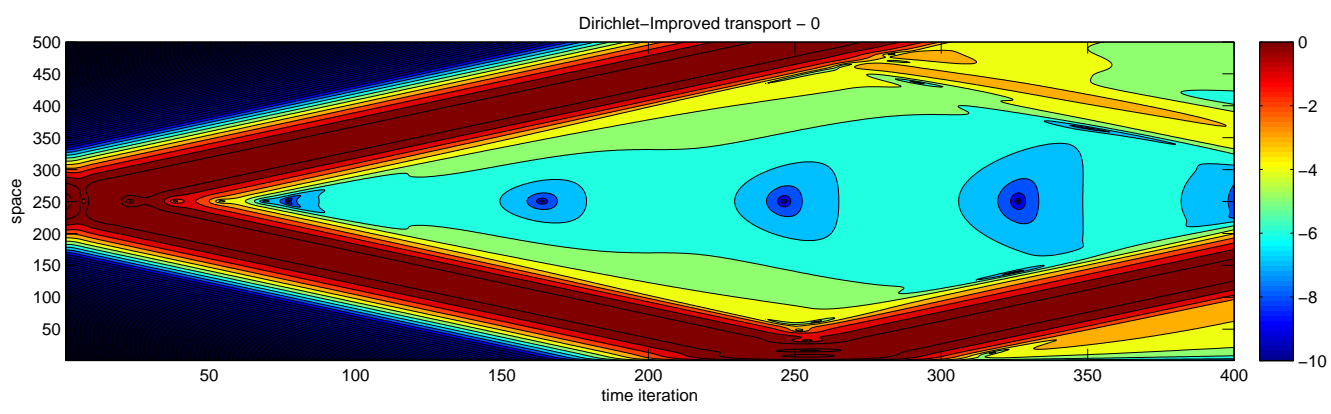

Figure 5: Laser-Klein-Gordon with Dirichlet (left) and improved transport at order 0 (right) boundary conditions

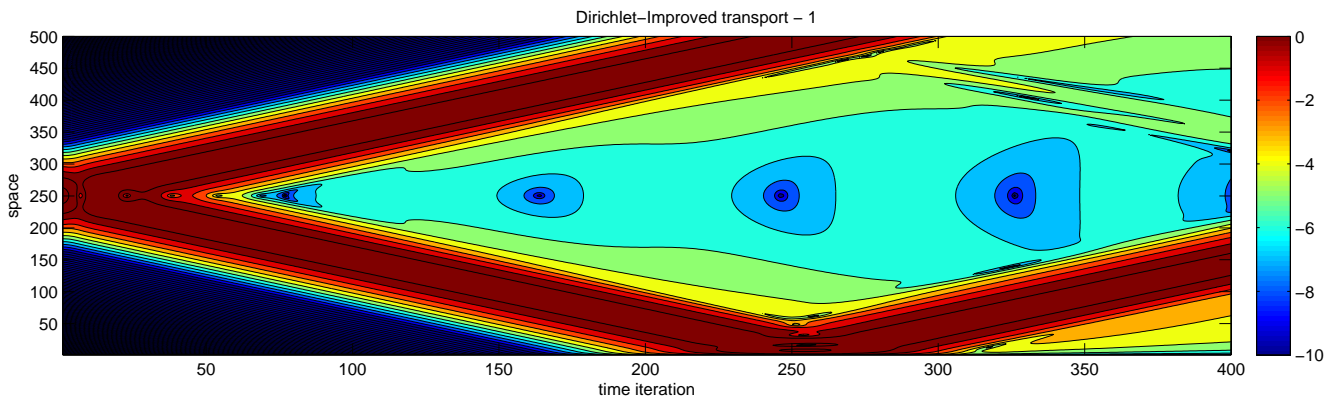

Figure 6: Laser-Klein-Gordon with Dirichlet (left) and improved transport at order -1 (right) boundary conditions

interior scheme in the laser-free case is standard. Denoting by $g$ the amplificator factor, and using the usual notations [27], we get for all $\theta \in[-\pi, \pi)$

$$
g^{2}(\theta)-2(1-\beta(\theta)) g(\theta)+1=0
$$

with

$$
\beta(\theta)=2 c^{2} \frac{\Delta t^{2}}{\Delta x^{2}} \sin ^{2}(\theta / 2)-\frac{\Delta t^{2} m^{2} c^{4}}{2} .
$$


Stability is ensured when the roots $g_{1}, g_{2}$ of this characteristic equations are less or equal to 1 , for all $\theta \in[-\pi, \pi)$. It is easy to prove that the scheme is stable provided that $\beta(\theta) \leqslant 2$ for all $\theta$, that is $\Delta t$ satisfies

$$
\Delta t \leqslant \frac{\Delta x}{c \sqrt{1+\frac{m^{2} c^{2}}{4} \Delta x^{2}} .}
$$

Stability for the interior scheme: laser-TDKGE. In the laser-KG case. In that case, we expect the time step to be time-dependent, as the equation equation involves a timedependent laser field. The characteristic equation in $g$ writes:

$$
g^{2}(\theta)-2 \alpha_{n}\left(1-\beta_{n}(\theta)\right) g(\theta)+\frac{\bar{\alpha}_{n}}{\alpha_{n}}=0
$$

with

$$
\alpha_{n}=\left(1+\frac{\Delta t_{n} A_{j}^{n}}{2}\right)^{-1}
$$

and

$$
\beta_{n}(\theta)=2 \frac{c^{2} \Delta t_{n}^{2} \sin ^{2}(\theta / 2)}{\Delta x^{2}}+\Delta t_{n}^{2} \frac{C_{j}^{n}}{2}-i \Delta t_{n}^{2} B_{j}^{n} \frac{\sin (\theta)}{\Delta x}
$$

or explicitely

$$
\begin{aligned}
\beta_{n}(\theta)= & \Delta t_{n}^{2} \frac{-i e \partial_{t} V\left(x_{j}, t_{n}\right)-i c^{2} \partial_{x} A_{x}\left(x_{j}, t_{n}\right)+e^{2} V^{2}\left(x_{j}, t_{n}\right)-c^{2} e^{2} A_{x}^{2}\left(x_{j}, t_{n}\right)-m^{2} c^{4}}{2} \\
& +2 \frac{c^{2} \Delta t_{n}^{2} \sin ^{2}(\theta / 2)}{\Delta x^{2}}-\Delta t_{n}^{2} e A_{x}\left(x_{j}, t_{n}\right) \frac{\sin (\theta)}{\Delta x} .
\end{aligned}
$$

or

$$
\begin{aligned}
\beta_{n}(\theta)= & \frac{2 c^{2} \Delta t_{n}^{2}}{\Delta x^{2}}\left(\frac{\Delta x^{2}\left(-i e \partial_{t} V\left(x_{j}, t_{n}\right)-i c^{2} \partial_{x} A_{x}\left(x_{j}, t_{n}\right)+e^{2} V^{2}\left(x_{j}, t_{n}\right)-c^{2} e^{2} A_{x}^{2}\left(x_{j}, t_{n}\right)-m^{2} c^{4}\right)}{4 c^{2}}\right. \\
& \left.+\sin ^{2}(\theta / 2)-\frac{\Delta x e A_{x}\left(x_{j}, t_{n}\right) \sin (\theta)}{2 c^{2}}\right) .
\end{aligned}
$$

Again the stability condition can be written as a function of $\beta_{n}$ using

$$
\left|g_{1}(\theta) g_{2}(\theta)\right|^{2}=\left|\frac{\bar{\alpha}_{n}}{\alpha_{n}}\right|^{2}=1
$$

and $g_{1}(\theta)+g_{2}(\theta)=2 \alpha_{n}(1-\beta(\theta))$. According to (41), the product of the root modulus is equal to 1 . We also assume that there exist $M_{1,2}$ positive and bounded constants such that $\|V\|_{\infty} \leqslant M_{1}$ and $\left\|A_{x}\right\|_{\infty} \leqslant M_{2}$. The roots are given by

$$
g_{1,2}(\theta)=\left(1-\beta_{n}(\theta)\right)\left[\alpha_{n} \pm \sqrt{\alpha_{n}\left(\alpha_{n}-1\right)}\right]
$$


and must satisfy $\left|g_{1,2}(\theta)\right| \leqslant 1$ for all $\theta$. Numerically, a stability condition can easily be determined at each time step, $\Delta t_{t} \leqslant \Delta t_{n}^{(i n t)}$. For instance, assuming $V=\partial_{x} A_{x}=0$, then $\alpha_{n}=1$ and the scheme is stable provided $\beta_{n} \leqslant 2$, for all $\theta$, that is:

$$
\beta_{n}(\theta) \leqslant\left|\beta_{n}(\theta)\right| \leqslant \frac{2 c^{2} \Delta t_{n}^{2}}{\Delta x^{2}}\left(1+\frac{\Delta x^{2}\left(c^{2} e^{2} M_{2}^{2}+m^{2} c^{4}\right)}{4 c^{2}}+\frac{\Delta x e M_{2}}{2 c^{2}}\right) \leqslant 2 .
$$

We conclude that in that simplified situation $\ell^{2}$-stability is ensured provided that

$$
\Delta t_{n} \leqslant \Delta t_{n}^{(\mathrm{int})}:=\frac{\Delta x}{c} \frac{1}{\sqrt{1+\frac{e M_{2}}{2 c^{2}} \Delta x+\Delta x^{2}\left(\frac{e^{2} M_{2}^{2}}{4}+\frac{m^{2} c^{2}}{4}\right)}} .
$$

Discretization of the boundary conditions. The discretization which is proposed is done according the boundary conditions derived in Proposition 2.1:

$$
\varphi_{N}^{n+1}=\varphi_{N}^{n}-\frac{c \Delta t_{n}}{\Delta x}\left(\varphi_{N}^{n}-\varphi_{N-1}^{n}\right)-i \Delta t_{n} \frac{c e}{2}\left(V_{N}^{n}-A_{x, N}^{n}\right) \varphi_{N}^{n}-\Delta t_{n} K_{N}^{n} \sum_{k=0}^{n} \Delta t_{k} \varphi_{N}^{k}
$$

We can rewrite this equation

$$
\varphi_{N}^{n+1}=L_{N}^{n} \varphi_{N}^{n}+M_{N}^{n} \varphi_{N-1}^{n}-\Delta t_{n} K_{N}^{n} \sum_{k=0}^{n-1} \Delta t_{k} \varphi_{N}^{k}
$$

where we have set

$$
\left\{\begin{aligned}
K_{N}^{n} & :=\frac{1}{4}\left(2 O_{D, N}^{n}-O_{F, N}^{n}-2 \mathrm{i} O_{G_{x}, N}^{n}+2 \mathrm{i} O_{G_{t}, N}^{n}\right) \\
L_{N}^{n} & :=1-\frac{c \Delta t_{n}}{\Delta x}-\Delta t_{n}^{2} K_{N}^{n}-\mathrm{i} \Delta t_{n} \frac{c e}{2}\left(V_{N}^{n}-A_{x, N}^{n}\right) \\
M_{N}^{n} & :=\frac{c \Delta t_{n}}{\Delta x}
\end{aligned}\right.
$$

As for all $n \geqslant 1, \varphi_{N}^{n}$ does not appear in the interior scheme, the stability analysis can be done independently at the boundary. We need to show that

$$
\Delta x\left|\varphi_{N}^{n+1}\right|^{2} \leqslant \Delta x \sum_{j=1}^{N}\left|\varphi_{j}^{n}\right|^{2}
$$

which is trivially satisfied if $\Delta t_{n} \leqslant \Delta t_{n}^{(a)}$ for $\Delta t_{n}^{(a)}$ ensuring simultaneously that

$$
\left|K_{N}^{n}\right| \leqslant 1,\left|L_{N}^{n}\right| \leqslant 1,\left|M_{N}^{n}\right| \leqslant 1
$$

Similarly, the scheme is stable for $\Delta t^{n} \leqslant \Delta t_{n}^{(-a)}$. 
Remark 5.1. Boundary conditions can also be derived following Section 2.1.2 and approximated in the spirit of [7]. Set

$$
Z:=-\partial_{t}^{2}+e^{2} V^{2}-c^{2} e^{2} A_{x}^{2}-m^{2} c^{4} .
$$

The $A B C$ can formally be written at $+a$

$$
c \partial_{x}-\mathrm{i} \sqrt{Z}-\mathrm{i} \frac{c e A_{x}}{2} .
$$

Padé's approximants for $k=1, \cdots, m$ for $m \geqslant 1$, write

$$
a_{k}^{m}=e^{\mathrm{i} \alpha / 2}\left(m \cos ^{2}\left[\frac{(2 k-1) \pi}{4 m}\right]\right)^{-1}, d_{k}^{m}=e^{\mathrm{i} \alpha} \tan ^{2}\left[\frac{(2 k-1) \pi}{4 m}\right] \alpha \in \mathbb{R}
$$

and as a consequence, we have the classical approximation:

$$
\sqrt{Z} \sim \sum_{k=1}^{m} a_{k}^{m}-\sum_{k=1}^{m} a_{k}^{m} d_{k}^{m}\left(Z+d_{k}^{m}\right)^{-1} .
$$

Set $v_{k}^{n}:=\left(Z+d_{k}^{m}\right)^{-1} \varphi^{n}$, that is $\left(Z+d_{k}^{m}\right) v_{k}^{n}=\varphi^{n}$. Then, at $x=x_{N}=a$ with $N \in \mathbb{N}$ such that $a=N \Delta x$

$$
c \partial_{x} \varphi_{N}^{n+1}-\mathrm{i} \sum_{k=1}^{m} a_{k} \varphi_{N}^{n+1}+\frac{i}{2} \sum_{k=1}^{m} a_{k}^{m} d_{k}^{m} v_{k}^{n+1}+\frac{i}{2} \sum_{k=1}^{m} a_{k}^{m} d_{k}^{m} v_{k}^{n}-i \frac{c e A_{x}^{n}}{2} \varphi_{N}^{n}=0
$$

where

$$
v_{k}^{n+1}=\frac{v_{k}^{n}\left(2-\frac{\Delta t_{n}^{2}}{2}\left(M_{k}^{n}-d_{k}^{m}\right)-\mathrm{i} e V_{N}^{n}\right)-v_{k}^{n-1}\left(1-\mathrm{i} e V_{N}^{n}\right)-\Delta t_{n}^{2} \varphi_{N}^{n+1}}{1+\frac{\Delta t_{n}^{2}}{2}\left(M_{k}^{n}-d_{k}^{m}\right)}
$$

where $M_{N}^{n}=m^{2} c^{4}-e^{2}\left(V_{N}^{n}\right)^{2}+e^{2} c^{2}\left(A_{x, N}^{n}\right)^{2} . A_{x, N}^{n}$ and $V_{N}^{n}$ denote $A_{x}\left(a, t_{n}\right)$ and $V\left(a, t_{n}\right)$. Finally, we approximate $\partial_{x} \varphi^{n+1}$ at a by $\Delta x\left(\varphi_{N}^{n+1}-\varphi_{N-1}^{n+1}\right)$, where $\varphi_{N-1}^{n+1}$ is computed by the interior scheme. In order to illustrate this approach we have solved the laser-free TDKGE on $(-10,10)$ with $c=m=1$, see Fig. 7 . The following numerical data are chosen: $N=400$ grid points, $m=100$ (for which convergence is reached) and $\alpha=-\pi / 4$.

Conclusion: stability of the numerical scheme. We can conclude that, from the interior scheme analysis that for $\Delta t_{n} \leqslant \Delta t_{n}^{(i n t)}$

$$
\Delta x \sum_{j=2}^{N-1}\left|\varphi_{j}^{n+1}\right|^{2} \leqslant \Delta x \sum_{j=2}^{N-1}\left|\varphi_{j}^{n}\right|^{2}
$$

and from the exterior scheme analysis that for $\Delta t_{n} \leqslant \Delta t_{n}^{(a)}$ and $\Delta t_{n} \leqslant \Delta t_{n}^{(-a)}$

$$
\Delta x\left|\varphi_{N}^{n+1}\right|^{2} \leqslant \Delta x\left|\varphi_{N}^{n}\right|^{2}, \quad \Delta x\left|\varphi_{1}^{n+1}\right|^{2} \leqslant \Delta x\left|\varphi_{1}^{n}\right|^{2} .
$$


Then for $\Delta t_{n} \leqslant \min \left(\Delta t_{n}^{(\mathrm{int})}, \Delta t_{n}^{(-a)}, \Delta t_{n}^{(a)}\right)$ the scheme is $\ell^{2}-$ stable, that is

$$
\Delta x \sum_{j=1}^{N}\left|\varphi_{j}^{n+1}\right|^{2} \leqslant \Delta x \sum_{j=1}^{N}\left|\varphi_{j}^{n}\right|^{2}
$$

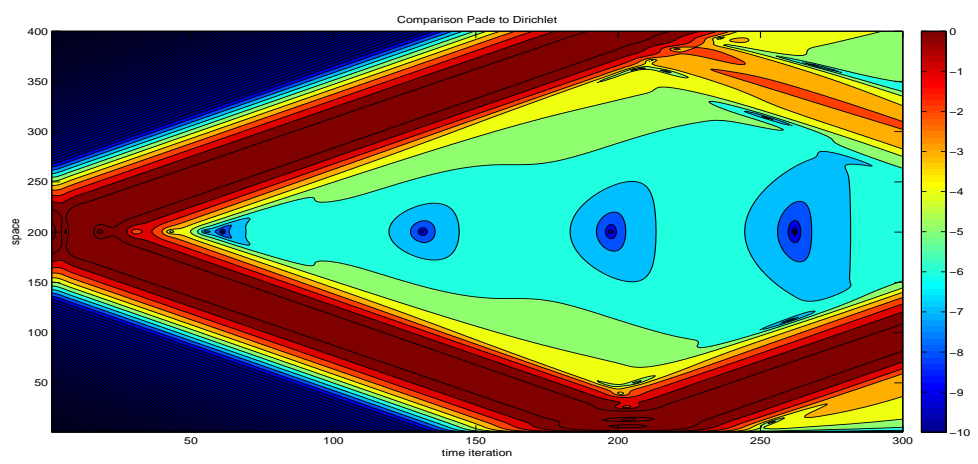

Figure 7: Klein-Gordon with Dirichlet (left) and Padé (right) boundary conditions

\subsection{Discretization for TDDE}

In this section, we propose a simple numerical illustrations of the derived boundary conditions for one-dimensional TDDE. The quantity which is represented here is again $\left(t, x, \log \left|\psi_{1}(x, t)\right|\right)$ which enlights the reflections at the domain boundary. We first rewrite $P_{D}$ in the form

$$
P_{D}=i \partial_{t}-i A \partial_{x}+B m c^{2}
$$

where

$$
A=\left(\begin{array}{cc}
c & 0 \\
0 & -c
\end{array}\right), \quad B=\mathrm{i}\left(\begin{array}{cc}
0 & m c^{2} \\
-m c^{2} & 0
\end{array}\right)
$$

A simple and natural condition to impose at $\pm a$ is $\left(\partial_{t} \pm c \partial_{x}\right) \psi_{1,2}=0$. We call such as condition a transport boundary condition, and corresponds in fact to order 0 condition in Proposition (3.3). We can easily check that for a $\mathrm{CFL}=1$ upwind scheme, Dirichlet's boundary condition (solution set to zero at the boundary) is equivalent to impose this transport condition. Initially, we set:

$\psi_{1}(x, 0)=\exp \left(-\frac{x^{2}}{\delta^{2}}\right) \exp \left(i k_{0} x\right), \psi_{2}(x, 0)=\frac{c k_{0}}{m c^{2}+\sqrt{m^{2} c^{4}+c^{2} k_{0}^{2}}} \exp \left(-\frac{x^{2}}{\delta^{2}}\right) \exp \left(i k_{0} x\right)$

where $c=1, k_{0}=-50, \delta=0.5, m=1$. The numerical domain is $(-a, a)$ with this time $a=5$. The final time is $T=10$ and $\Delta t=\Delta x=1 / 60$. One-dimensional ABC can easily be 
deduced from Proposition 3.2.

The numerical scheme we consider is as follows. We denote by $\left(\phi_{j}^{n}, \psi_{j}^{n}\right)$ and approximation of the exact two-spinors at $\left(x_{j}, t_{n}\right),\left(\psi_{1}\left(x_{j}, t_{n}\right), \psi_{2}\left(x_{j}, t_{n}\right)\right)$. The numerical scheme writes

$$
\left\{\begin{array}{l}
\phi_{j}^{n+1}=\phi_{j}^{n}+c \frac{\Delta t}{\Delta x}\left(\phi_{j+1}^{n}-\phi_{j}^{n}\right)+\Delta t m c^{2} \psi_{j}^{n} \\
\psi_{j}^{n+1}=\psi_{j}^{n}-c \frac{\Delta t}{\Delta x}\left(\psi_{j}^{n}-\psi_{j-1}^{n}\right)-\Delta t m c^{2} \phi_{j}^{n} .
\end{array}\right.
$$

Stability for the interior scheme: laser-free-TDDE. One denotes $g_{1}(\theta), g_{2}(\theta)$ the amplification factors for $\phi$ and $\psi$, with $\theta \in[-\pi, \pi)$, and $\xi \Delta x$, where $\xi$ is the dual variable to $x$.

$$
\left\{\begin{aligned}
g_{1}^{n+1}(\theta) & =\left(1+c \frac{\Delta t}{\Delta x}\left(e^{\mathrm{i} \theta}+1\right)\right) g_{1}^{n}(\theta)+\Delta t m c^{2} g_{2}^{n}(\theta) \\
g_{2}^{n+1}(\theta) & =\left(1-c \frac{\Delta t}{\Delta x}\left(1-e^{-\mathrm{i} \theta}\right)\right) g_{2}^{n}(\theta)-\Delta t m c^{2} g_{1}^{n}(\theta) .
\end{aligned}\right.
$$

A necessary and sufficient condition for stability is $\left|g_{1}(\theta)\right|,\left|g_{2}(\theta)\right|$ are less than 1 for any $\theta \in[-\pi, \pi)$. That is $g_{1,2}$ are roots of the following equation with $\nu=c \Delta t / \Delta x$,

$$
g^{2}(\theta)-2\left(1-2 \nu \sin ^{2}(\theta / 2)\right) g(\theta)-4 \nu \sin ^{2}(\theta / 2)+4 \nu^{2} \sin ^{2}(\theta / 2)+1+\Delta t^{2} m^{2} c^{4}=0 .
$$

We deduce $g_{1}(\theta) g_{2}(\theta)=1+\Delta t^{2} m^{2} c^{4}-4 \nu \sin ^{2}(\theta / 2)(1-\nu)$ and $g_{1}(\theta)+g_{2}(\theta)=2(1-$ $2 \nu \sin ^{2}(\theta / 2)$. To ensure stability, we naturally need $\left|g_{1}(\theta) g_{2}(\theta)\right| \leqslant 1$ for all $\theta \in[-\pi, \pi)$. Then a sufficient condition for $\ell^{2}$-stability writes

$$
c \frac{\Delta t}{\Delta x} \leqslant 1
$$

Stability at the boundary condition: laser-free-TDDE. The implementation of the above boundary conditions for TDDE is straightforward. We can indeed rearrange the scheme. For $j \neq 0$ and $j \neq N$

$$
\left\{\begin{array}{l}
\psi_{1, j}^{n+1}=\frac{\psi_{1, j+1}^{n}+\psi_{2, j+1}^{n}+\psi_{1, j-1}^{n}-\psi_{2, j-1}^{n}}{2}+\Delta t m c^{2} \frac{\psi_{1, j}^{n}+\psi_{2, j}^{n}}{2} \\
\psi_{2, j}^{n+1}=\frac{\psi_{1, j+1}^{n}+\psi_{2, j+1}^{n}-\psi_{1, j-1}^{n}+\psi_{2, j-1}^{n}}{2}-\Delta t m c^{2} \frac{\psi_{1, j}^{n}+\psi_{2, j}^{n}}{2}
\end{array}\right.
$$

At order 0 , we impose $\psi_{1,0}^{n}-\psi_{2,0}^{n}=0$ at the left boundary, and $\psi_{1, N}^{n}+\psi_{2, N}^{n}=0$ at the right one. Remark that for the very particular interior scheme which is considered here, Dirichlet's boundary conditions are equivalent to this order 0 condition (transport). At the next order -1 is simply replaced by a Riemann-Liouville integrals. Stability is again trivially satisfied under CFL condition. 

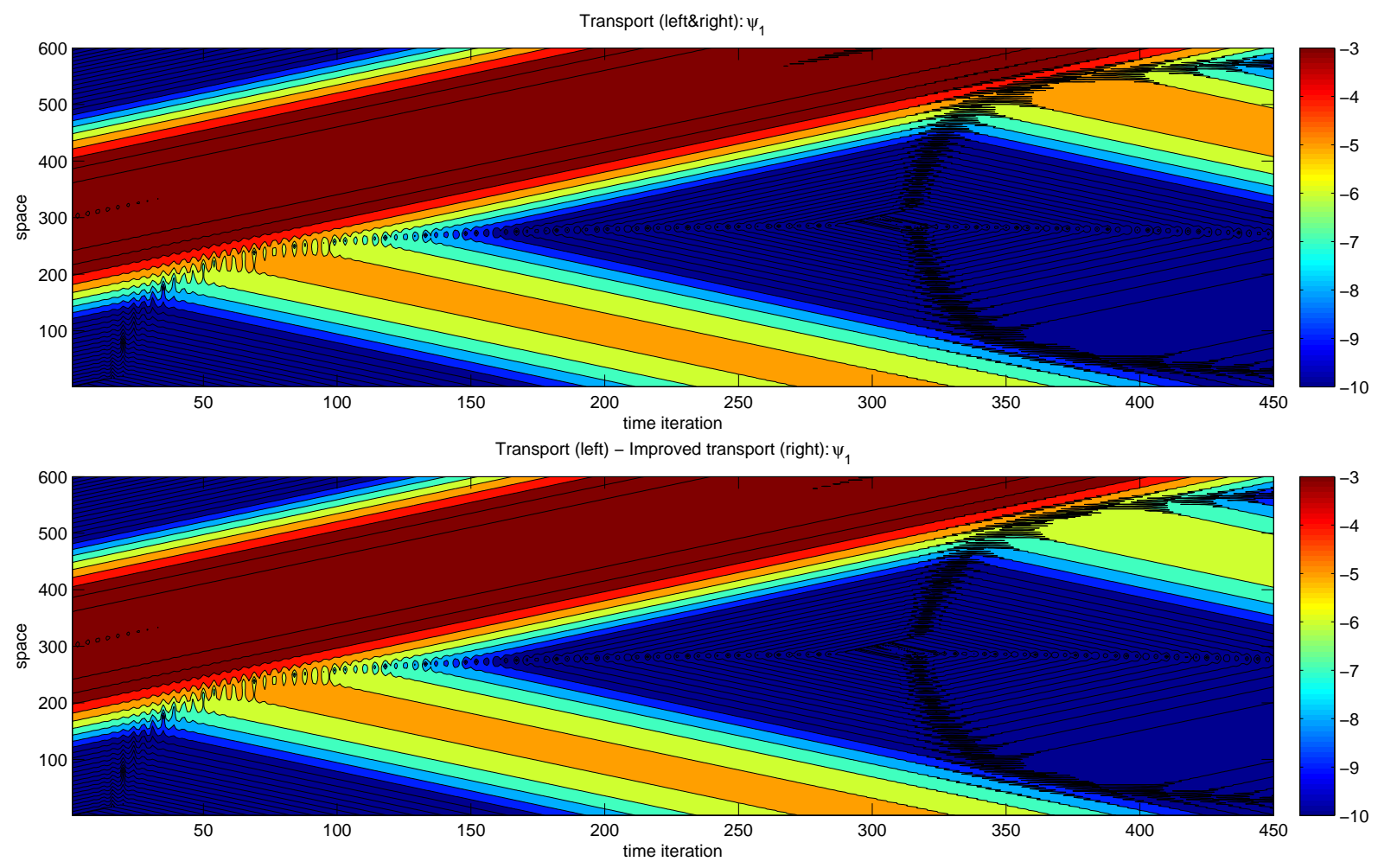

Figure 8: $\mathrm{ABC}$ for $\log \left(\psi_{1}\right)$ a) order $\left.0, \mathrm{~b}\right)$ order -1

\section{Conclusion}

This paper objective was to rigorously derive absorbing boundary conditions for quantum relativistic equations. Both Klein-Gordon and Dirac equations were considered for quantum particle subject to classical electromagnetic fields. Using usul microlocal analysis tools, sequences of more and more accurate absorbing boundary conditions were derived for these equations. Simple numerical discretizations and simulations were proposed and analysez to illustrate the accuracy of these ABC. Several analytical questions still remain to be addressed:

- The well-posedness of the mixed problem

$$
\begin{cases}P_{D, K G} \mathbf{w}_{D, K G}=\mathbf{0} & \text { on } \Omega_{D, K G} \times[0, T] \\ \mathcal{B}_{D, K G} \mathbf{w}_{D, K G}=\mathbf{0} & \text { on } \partial \Omega_{D, K G} \times[0, T] \\ \mathbf{w}_{D, K G}(\cdot, 0)=\mathbf{u}_{D, K G}^{0} & \text { on } \Omega_{D, K G}\end{cases}
$$

where the index $D$ stands for Dirac and $K G$ for Klein-Gordon, is naturally the very first important question. 

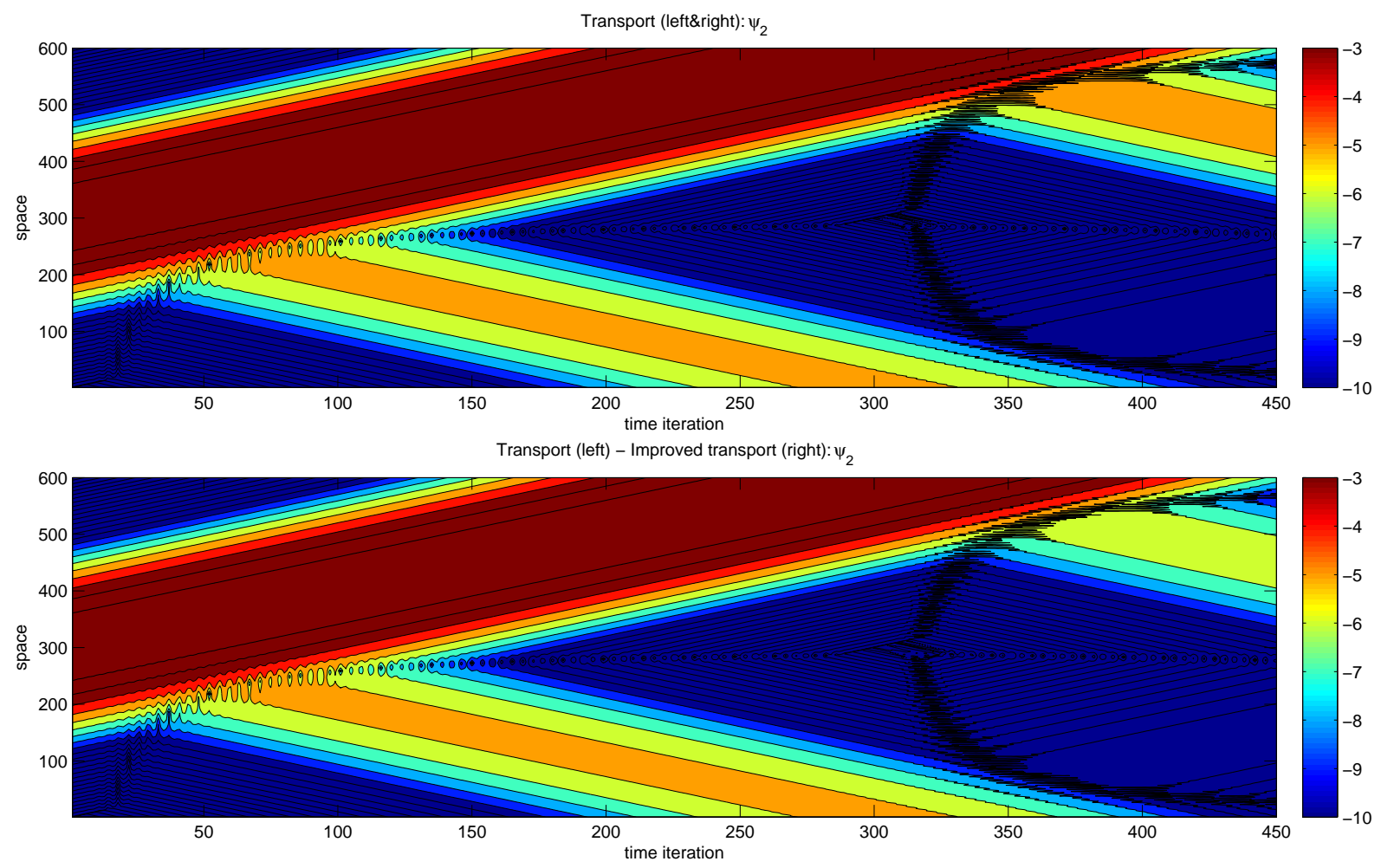

Figure 9: $\mathrm{ABC}$ for $\log \left(\psi_{2}\right)$ a) order $0, \mathrm{~b}$ ) order -1

- The error analysis which consists of estimating $\left\|\mathbf{u}_{D, K G}-\mathbf{w}_{D, K G}\right\|_{H\left(\Omega_{D, K G}\right)}$ in the appropriate Hilbert space $H\left(\Omega_{D, K G}\right)$, where $\mathbf{u}_{D, K G}$ satisfies

$$
\left\{\begin{array}{l}
P_{D, K G} \mathbf{u}_{D, K G}=\mathbf{0} \quad \text { on } \mathbb{R}^{2} \times[0, T] \\
\mathbf{u}_{D, K G}(\cdot, 0)=\mathbf{u}_{D, K G}^{0}
\end{array}\right.
$$

is also a fundamental question from a practical point of view. These questions were for instance treated in [8] for Maxwell's equations.

From a computational point of view, several problems have also to be considered.

- Although it is easy to derive accurate numerical schemes for the TDKGE and TDDE on unbounded domains, the inclusion of boundary conditions, especially for spectral, finite element or implicit finite volume and difference schemes which necessitate the solving of linear systems, may introduce some computational difficulties. As an example, the numerical approximation of TDSE with absorbing boundary conditions derived in a similar way, necessitates a very subtle discretization to maintain the unconditional $\ell^{2}$-stability of a simple Crank-Nicolson type scheme in 1-d [6]. In addition, the conditioning of matrices involved in the linear systems may increase [21]. 
- A key problem, which is naturally coupled with the ones described above, is the derivation of accurate and efficient discretization of the pseudo-differential operators involved in the derived ABC. Some of them are nonlocal in time and in space such as the Riemann-Liouville operators (21) which complicates their approximation.

- The implementation of these conditions in numerical codes for the computation of multidimensional TDKGE and TDDE is naturally the final objective. For instance, production particle/anti-particle paires using intense and short laser pulses is a very active research area [14], [13]. However, the corresponding simulations, based on laserparticle TDDE, necessitate huge computational efforts [12], [11]. Accurate and efficient ABC could reduce drastically the computational cost of these simulations.

These questions will be treated in forthcoming papers and will justify the use of such absorbing boundary conditions.

[1] S. Alinhac and P. Gérard. Pseudo-differential operators and the Nash-Moser theorem, volume 82 of Graduate Studies in Mathematics. American Mathematical Society, Providence, RI, 2007. Translated from the 1991 French original by Stephen S. Wilson.

[2] X. Antoine and H. Barucq. Microlocal diagonalization of strictly hyperbolic pseudodifferential systems and application to the design of radiation conditions in electromagnetism. SIAM J. Appl. Math., 61(6):1877-1905 (electronic), 2001.

[3] X. Antoine and H. Barucq. On the construction of approximate boundary conditions for solving the interior problem of the acoustic scattering transmission problem. In Domain decomposition methods in science and engineering, volume 40 of Lect. Notes Comput. Sci. Eng., pages 133-140. Springer, Berlin, 2005.

[4] X. Antoine, H. Barucq, and A. Bendali. Bayliss-Turkel-like radiation conditions on surfaces of arbitrary shape. J. Math. Anal. Appl., 229(1):184-211, 1999.

[5] X. Antoine and C. Besse. Construction, structure and asymptotic approximations of a microdifferential transparent boundary condition for the linear Schrödinger equation. J. Math. Pures Appl. (9), 80(7):701-738, 2001.

[6] X. Antoine and C. Besse. Unconditionally stable discretization schemes of non-reflecting boundary conditions for the one-dimensional Schrödinger equation. J. Comput. Phys., 188(1):157-175, 2003.

[7] X. Antoine, C. Besse, and P. Klein. Absorbing boundary conditions for the onedimensional Schrödinger equation with an exterior repulsive potential. J. Comput. Phys., 228(2):312-335, 2009.

[8] H. Barucq and M. Fontes. Well-posedness and exponential stability of Maxwell-like systems coupled with strongly absorbing layers. J. Math. Pures Appl. (9), 87(3), 2007. 
[9] R. Dautray and J.-L. Lions. Analyse mathématique et calcul numérique pour les sciences et les techniques. Vol. 5. INSTN: Collection Enseignement. [INSTN: Teaching Collection]. Masson, Paris, 1988. Spectre des opérateurs. [The operator spectrum], With the collaboration of Michel Artola, Michel Cessenat, Jean Michel Combes and Bruno Scheurer, Reprinted from the 1984 edition.

[10] B. Engquist and A. Majda. Absorbing boundary conditions for the numerical simulation of waves. Math. Comp., 31(139):629-651, 1977.

[11] F. Fillion-Gourdeau, E. L. De La Grandmaison, and A. D. Bandrauk. Relativistic ground state of diatomic molecules from the numerical solution of the dirac equation on parallel computers. Journal of Physics: Conference Series, 341(1), 2012.

[12] F. Fillion-Gourdeau, E. Lorin, and A. D. Bandrauk. Numerical solution of the timedependent Dirac equation in coordinate space without fermion-doubling. Comput. Phys. Commun., 183(7):1403-1415, 2012.

[13] F. Fillion-Gourdeau, E. Lorin, and A. D. Bandrauk. Relativistic stark resonances in a simple exactly soluble model for a diatomic molecule. Journal of Physics A: Mathematical and Theoretical, 45(21), 2012. Cited By (since 1996): 1.

[14] F. Fillion-Gourdeau, E. Lorin, and A. D. Bandrauk. Resonantly enhanced pair production in a simple diatomic model. Physical Review Letters, 110(1), 2013.

[15] J.-M. Ghidaglia and F. Pascal. The normal flux method at the boundary for multidimensional finite volume approximations in CFD. Eur. J. Mech. B Fluids, 24(1):1-17, 2005.

[16] L. Halpern and J. Rauch. Error analysis for absorbing boundary conditions. Numer. Math., 51(4):459-467, 1987.

[17] L. Hörmander. Linear partial differential operators. Springer Verlag, Berlin, 1976.

[18] L. Hörmander. The analysis of linear partial differential operators. III. Classics in Mathematics. Springer, Berlin, 2007. Pseudo-differential operators.

[19] A. Iserles. On the numerical quadrature of highly-oscillating integrals. I. Fourier transforms. IMA J. Numer. Anal., 24(3):365-391, 2004.

[20] A. Iserles. On the numerical quadrature of highly-oscillating integrals. II. Irregular oscillators. IMA J. Numer. Anal., 25(1):25-44, 2005.

[21] R. Kechroud, X. Antoine, and A. Soulaïmani. Numerical accuracy of a Padé-type non-reflecting boundary condition for the finite element solution of acoustic scattering problems at high-frequency. Internat. J. Numer. Methods Engrg., 64(10):1275-1302, 2005. 
[22] R. Lascar. Propagation des singularités des solutions d'équations pseudo-différentielles quasi homogènes. Ann. Inst. Fourier (Grenoble), 27(2):vii-viii, 79-123, 1977.

[23] E. Lorin and A. Bandrauk. A simple and accurate mixed $P^{0}-Q^{1}$ solver for the MaxwellDirac equations. Nonlinear Anal. Real World Appl., 12(1):190-202, 2011.

[24] E. Lorin, S. Chelkowski, and A. D. Bandrauk. Mathematical modeling of boundary conditions for laser-molecule time-dependent Schrödinger equations and some aspects of their numerical computation - one-dimensional case. Numer. Methods Partial Differential Equations, 25(1):110-136, 2009.

[25] J.T. Mendon ça and A. Serbeto. Volkov solutions for relativistic quantum plasmas. Physical Review E - Statistical, Nonlinear, and Soft Matter Physics, 83(2), 2011.

[26] L. Nirenberg. Lectures on linear partial differential equations. American Mathematical Society, Providence, R.I., 1973.

[27] J. C. Strikwerda. Finite difference schemes and partial differential equations. Society for Industrial and Applied Mathematics (SIAM), Philadelphia, PA, second edition, 2004.

[28] M. E. Taylor. Partial differential equations III. Nonlinear equations, volume 117 of Applied Mathematical Sciences. Springer, New York, second edition, 2011.

[29] B. Thaller. The Dirac equation. Texts and Monographs in Physics. Springer-Verlag, Berlin, 1992.

[30] V. Vaibhav. Artificial boundary conditions for certain evolution PDEs with cubic nonlinearity for non-compactly supported initial data. J. Comput. Phys., 230(8):3205-3229, 2011. 